Florida International University FIU Digital Commons

$6-7-2013$

\title{
Aedes aegypti Pharate First Instar Aseasonal Quiescence Cues Anticipatory Plasticity with Implications for Urban Vector Ecology and Control
}

Mario H. Perez

Florida International Universty, perezmh@fiu.edu

DOI: $10.25148 /$ etd.FI13080702

Follow this and additional works at: https://digitalcommons.fiu.edu/etd

Part of the Life Sciences Commons

\section{Recommended Citation}

Perez, Mario H., "Aedes aegypti Pharate First Instar Aseasonal Quiescence Cues Anticipatory Plasticity with Implications for Urban Vector Ecology and Control" (2013). FIU Electronic Theses and Dissertations. 913.

https://digitalcommons.fiu.edu/etd/913 


\section{FLORIDA INTERNATIONAL UNIVERSITY}

Miami, Florida

AEDES AEGYPTI PHARATE FIRST INSTAR ASEASONAL QUIESCENCE CUES ANTICIPATORY PLASTICITY WITH IMPLICATIONS FOR URBAN VECTOR ECOLOGY AND CONTROL

A dissertation submitted in partial fulfillment of the

requirements for the degree of DOCTOR OF PHILOSOPHY

in

BIOLOGY

by

Mario H. Perez

2013 


\section{To: Dean Kenneth Furton}

College of Arts and Sciences

This dissertation, written by Mario H. Perez, and entitled Aedes aegypti Pharate First Instar Aseasonal Quiescence Cues Anticipatory Plasticity with Implications for Urban Vector Ecology and Control, having been approved in respect to style and intellectual content, is referred to you for judgment.

We have read this dissertation and recommend that it be approved.

$\begin{array}{r}\hline \text { Philip K. Stoddard } \\ \hline \text { Manuel A. Barbieri } \\ \hline \text { Martin Tracey } \\ \hline \text { Piero Gardinali } \\ \hline \text { Fernando G. Noriega, Major Professor }\end{array}$

Date of Defense: June 7, 2013

The dissertation of Mario H. Perez is approved.

\begin{tabular}{r}
\hline $\begin{array}{r}\text { Dean Kenneth Furton } \\
\text { College of Arts and Sciences }\end{array}$ \\
\hline Dean Lakshmi N. Reddi \\
University Graduate School
\end{tabular}

Florida International University, 2013 


\section{DEDICATION}

This dissertation is lovingly dedicated to my parents, Henry and Martha Perez, to my father for instilling in me a perpetual curiosity of the natural world and to my mother for her continued support, encouragement, and constant love. 


\section{ACKNOWLEDGMENTS}

I would like to thank all the members of the Noriega laboratory, both current and former; in particular, I would like to thank former research associates Dr. Salvador Hernandez Martinez who first introduced me to the world of insect physiology, Dr. Jaime G. Mayoral who first trained me in molecular biology and present research associate Dr. Marcela Nouzova for her detailed training and mindful observations with which I made a critical leap in my research. I would also like to thank Dr. Crisalejandra Rivera Perez for her perpetual feedback with a biochemical perspective and Dr. Mark Clifton for his sense of perspective and broad scope.

I would like to thank my major advisor Prof. Fernando G. Noriega for his patience as a mentor and for allowing me the freedom to explore a line of research of my own design and opening up his lab to me like a second home; my committee members Prof. Phillip K. Stoddard, Prof. Martin Tracey, Prof. Alejando Barbieri and Prof. Piero Gardinali for their feedback, critical analysis and general overall support.

I would like to thank the MBRS-RISE research fellowship (NIH/NIG-MS R25 GM061347) and the FIU Teaching Assistantship for their financial support throughout this dissertation along with the FIU MBRS RISE Biomedical Research Initiative to MHP.

I would like to acknowledge Elsevier for permission to reprint chapters four and five which originally appeared in the Journal of Insect Physiology, volume 58, issue 6, and volume 59, issue 3 respectively.

Finally, I would like to thank my undergraduate advisor Prof. William G. Wallace for inspiring in me the confidence to pursue a doctorate. 


\section{ABSTRACT OF THE DISSERTATION \\ AEDES AEGYPTI PHARATE FIRST INSTAR ASEASONAL QUIESCENCE CUES ANTICIPATORY PLASTICITY WITH IMPLICATIONS FOR URBAN VECTOR ECOLOGY AND CONTROL}

by

Mario H. Perez

Florida International University, 2013

Miami, Florida

Professor Fernando G. Noriega, Major Professor

The eggs of the dengue fever vector Aedes aegypti possess the ability to undergo an extended quiescence period hosting a fully developed first instar larvae within its chorion. As a result of this life history stage, pharate larvae can withstand months of dormancy inside the egg where they depend on stored reserves of maternal origin. This adaptation known as pharate first instar quiescence, allows $A$. aegypti to cope with fluctuations in water availability. An examination of this fundamental adaptation has shown that there are trade-offs associated with it.

Aedes aegypti mosquitoes are frequently associated with urban habitats that may contain metal pollution. My research has demonstrated that the duration of this quiescence and the extent of nutritional depletion associated with it affects the physiology and survival of larvae that hatch in a suboptimal habitat; nutrient reserves decrease during pharate first instar quiescence and alter subsequent larval and adult fitness. The duration of quiescence compromises metal tolerance physiology and is coupled to a decrease in metallothionein mRNA levels. My findings also indicate that even low levels of 
environmentally relevant larval metal stress alter the parameters that determine vector capacity.

My research has also demonstrated that extended pharate first instar quiescence can elicit a plastic response resulting in an adult phenotype distinct from adults reared from short quiescence eggs. Extended pharate first instar quiescence affects the performance and reproductive fitness of the adult female mosquito as well as the nutritional status of its progeny via maternal effects in an adaptive manner, i.e., anticipatory phenotypic plasticity results as a consequence of the duration of pharate first instar quiescence and alternative phenotypes may exist for this mosquito with quiescence serving as a cue possibly signaling the environmental conditions that follow a dry period. M findings may explain, in part, $A$. aegypti's success as a vector and its geographic distribution and have implications for its vector capacity and control. 


\section{TABLE OF CONTENTS}

CHAPTER PAGE

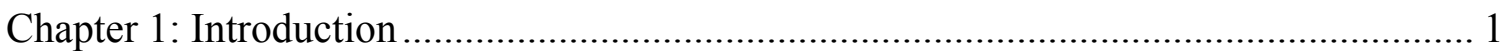

1.1 Aedes aegypti: Global distribution and success as a pathogen vector ...................... 1

1.2 Dissertation justification and objective .................................................................. 3

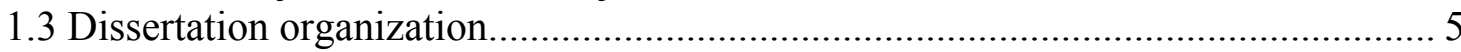

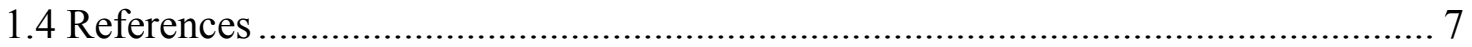

Chapter 2: A. aegypti: A combination of a distinct life history trait, plasticity and vector

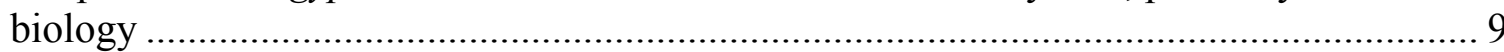

2.1 Diapause $v s$. quiescence in mosquitoes............................................................... 9

2.2 Anticipatory phenotypic plasticity - environment, development and genes ........... 12

2.2.1 Background and nomenclature …………………..................................... 12

2.2.2 Polyphenic development in insects............................................................ 18

2.2.3 Alternative phenotypes in mosquitoes........................................................... 28

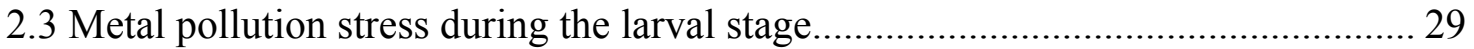

2.3.1 Vectorial consequences of larvae metal stress ………..................................... 32

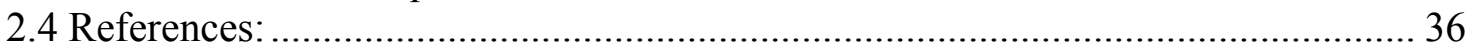

Chapter 3: The sub-lethal larval metal stress response of the Dengue Fever Mosquito... 43

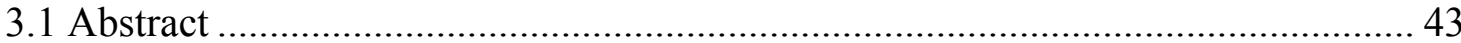

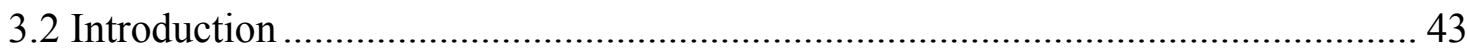

3.3 Materials and Methods ................................................................................... 46

3.3.1 Mosquito rearing and maintenance.............................................................. 46

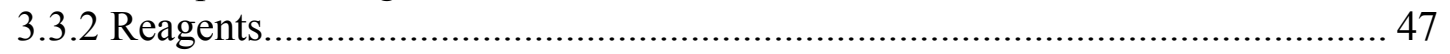

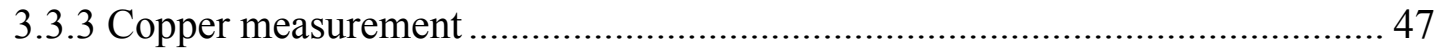

3.3.4 Images of first instars and adult tissues......................................................... 47

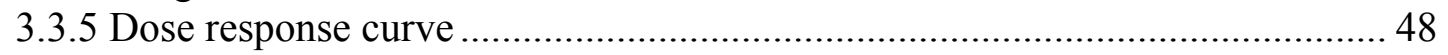

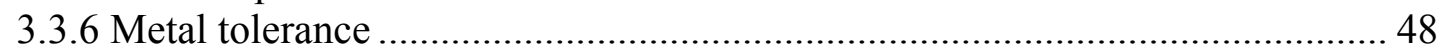

3.3.7 Quantification of total neutral lipids............................................................ 48

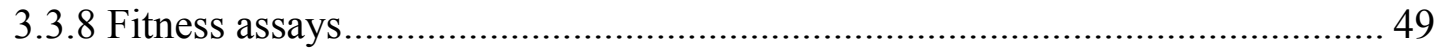

3.3.9 Quantification of follicles per ovary............................................................. 49

3.3.10 A. aegypti metallothionein (AaMtn) induction.............................................. 50

3.3.11 Midgut as a barrier to metal toxicity .......................................................... 50

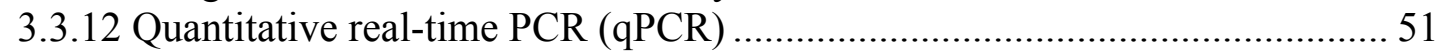

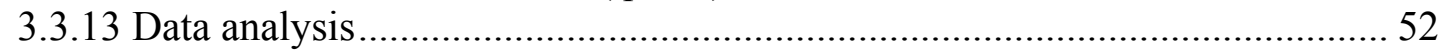

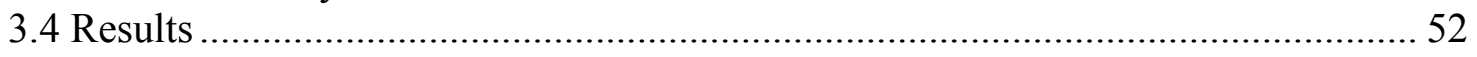

3.4.1 Larval metal stress altered larval and adult phenotypes .................................. 52

3.4.2 A. aegypti larvae were sensitive to metal stress................................................ 53

3.4.3 A. aegypti larvae exhibited tolerance........................................................... 53

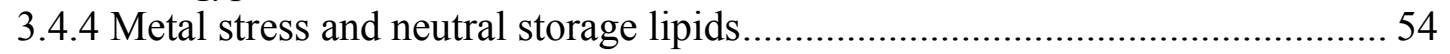

3.4.5 AaMtn was expressed predominately in the midgut of larvae ......................... 54

3.4.6 AaMtn transcription of first instars was highly dynamic .................................. 54

3.4.7 Larval metal stress is reflected in adult phenotype.......................................... 55 


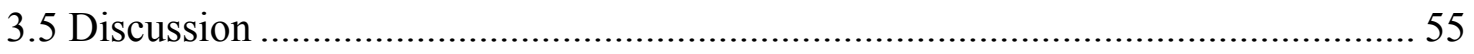

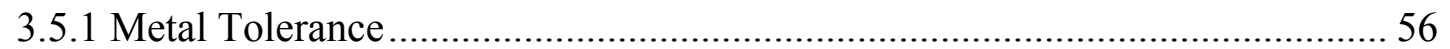

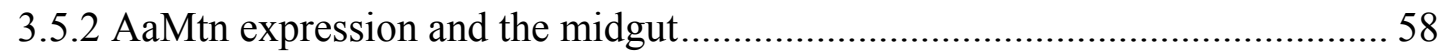

3.5.3 Implications for Vector Capacity ................................................................... 59

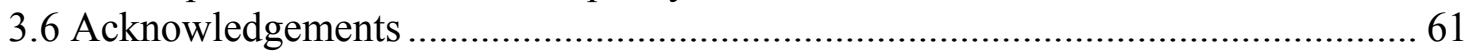

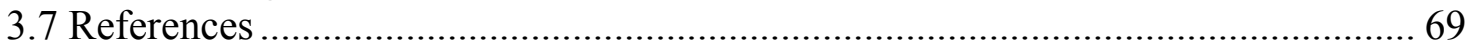

Chapter 4: Aedes aegypti pharate first instar quiescence affects larval fitness and metal

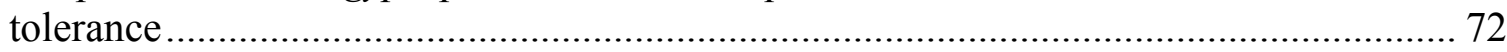

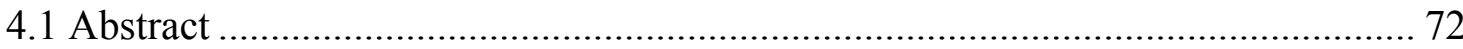

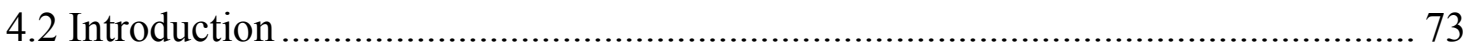

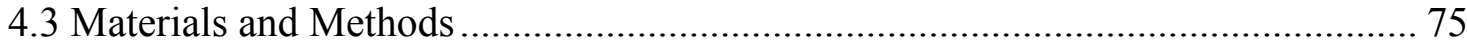

4.3.1 Mosquito rearing and maintenance........................................................... 75

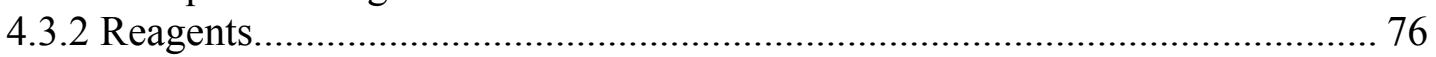

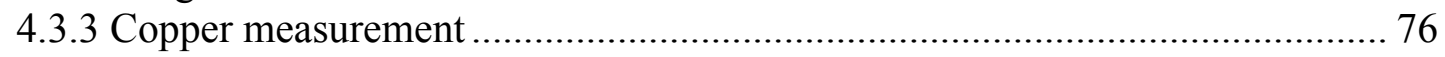

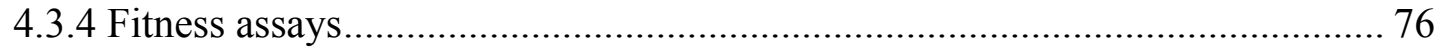

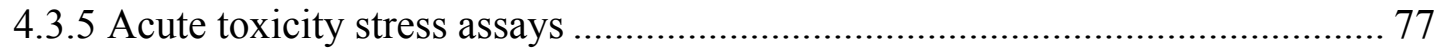

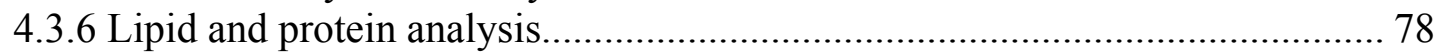

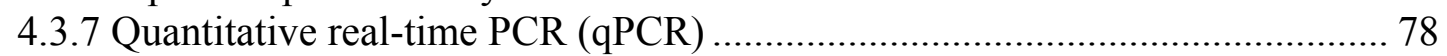

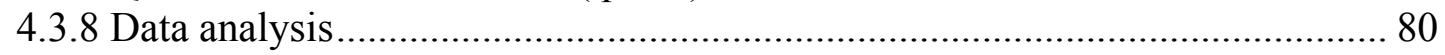

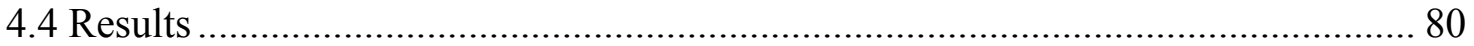

4.4.1 Effect of quiescence on first instar larval survivorship and growth ................. 80

4.4.2 Effect of quiescence on larval nutrient reserves .............................................. 80

4.4.3 Effect of quiescence on first instar larvae response to metal stress.................... 81

4.4.4 Effect of quiescence on metallothionein mRNA levels..................................... 81

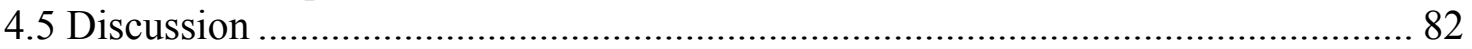

4.5.1 The duration of quiescence alters the nutritional status, growth rate and starvation tolerance of newly hatched larvae .......................................................... 82 4.5.2 The duration of quiescence alters the physiological response to metal stress in

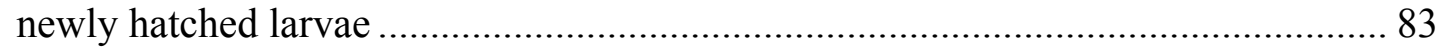

4.5.3 The duration of quiescence alters the molecular response to metal stress in

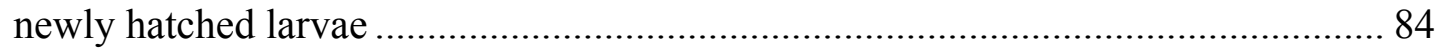

4.5.4 Implications for Vector Ecology and Vector Control ..................................... 86

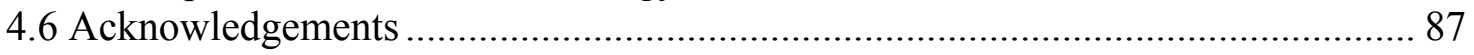

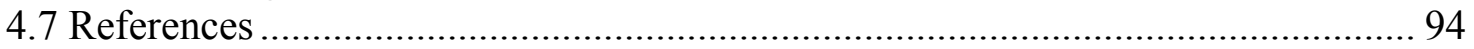

Chapter 5: Aedes aegypti pharate first instar quiescence: A case for anticipatory

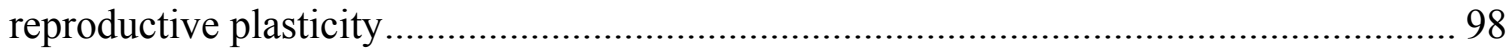

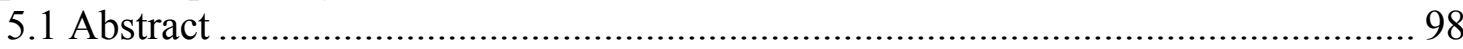

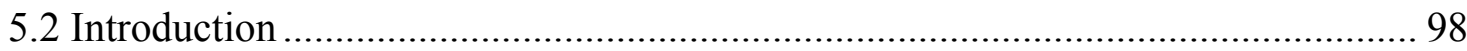

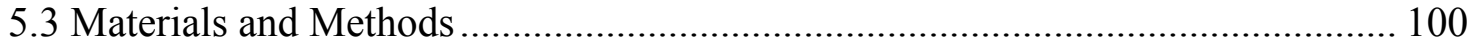

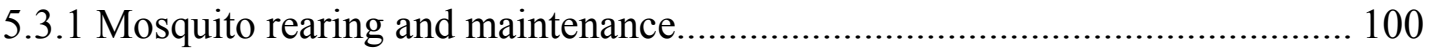

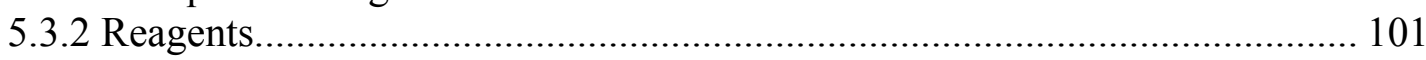

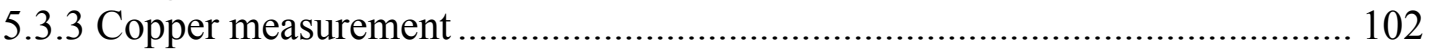

5.3.4 Fitness assays ...................................................................................... 102

5.3.5 Quantification of total neutral lipids.............................................................. 103 
5.3.6 Quantification of follicles per ovary............................................................. 103

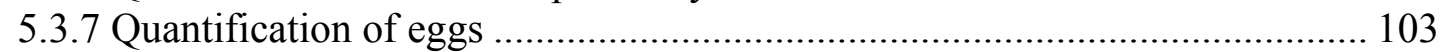

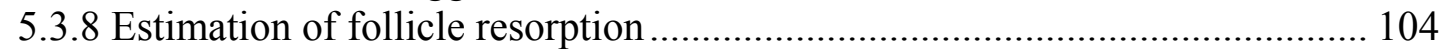

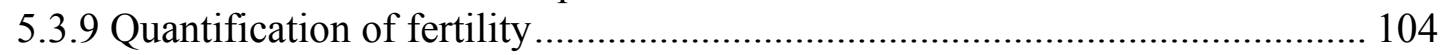

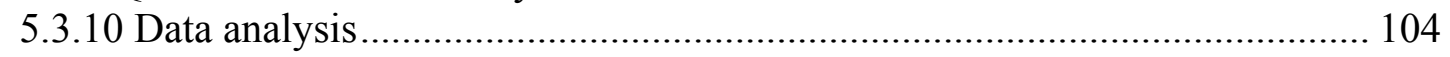

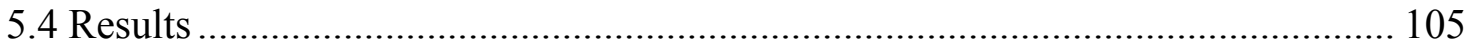

5.4.1 Extended quiescence altered female adult performance.................................. 105

5.4.2 Extended quiescence altered reproductive strategy ........................................ 106

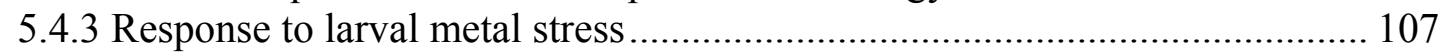

5.4.4 Extended quiescence produced maternal effects ........................................... 107

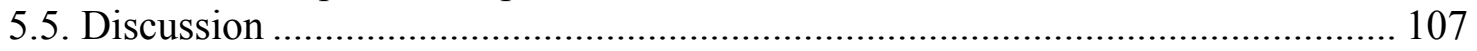

5.5.1 Quiescence and female adult performance.................................................. 108

5.5.3 Extended quiescence and maternal effects ................................................. 113

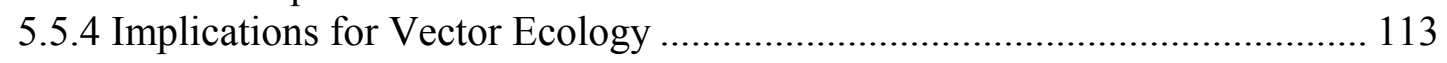

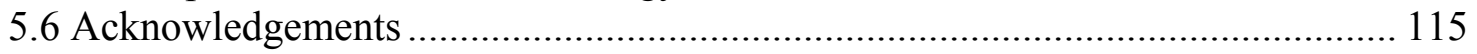

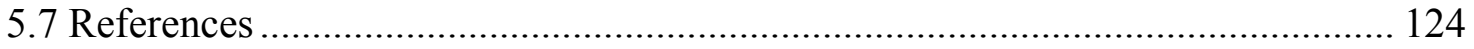

Chapter 6: Conclusions and Final Remarks.............................................................. 128

6.1 A. aegypti pharate first instar quiescence: A distinctive life history trait with tradeoffs and implications for urban vector biology ...................................................... 128

6.2 Vector Capacity and the larval environment of $A$. aegypti ................................. 132

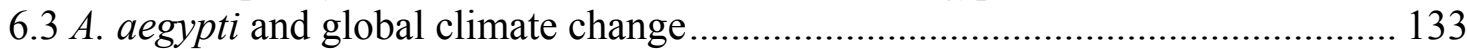

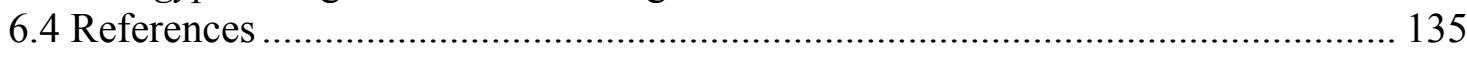

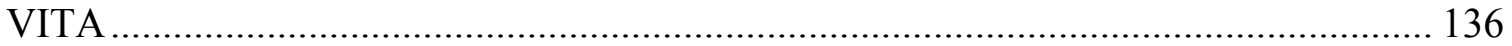




\section{LIST OF FIGURES}

FIGURE

PAGE

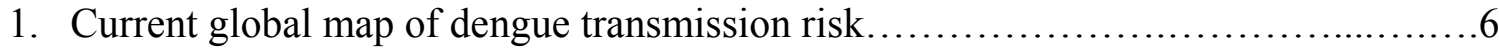

2. Polyphenisms in insects are usually contrasting and dramatic.......................34

3. A generalized schematic representation of the control mechanisms

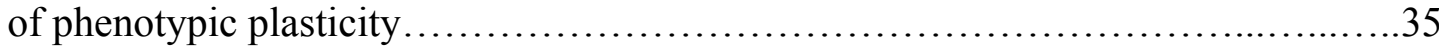

4. Low sub-lethal metal stress compromised larval and adult phenotypes..............62

5. Dose response curve for copper............................................63

6. A. aegypti 3rd instars exhibited tolerance....................................64

7. The nutritional cost of metal stress......................................65

8. AaMtn was expressed predominately in the midgut of larvae................................66

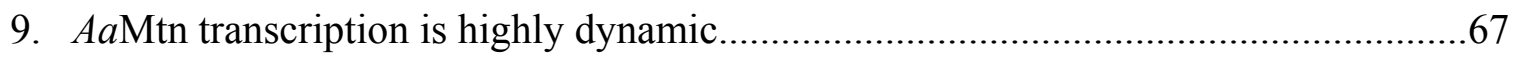

10. Larval metal stress is reflected in adult phenotype .............................................68

11. Larval development period in a clean environment.............................................. 88

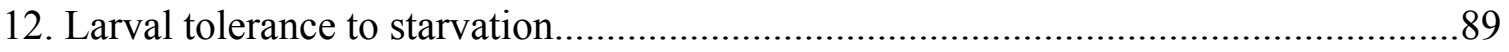

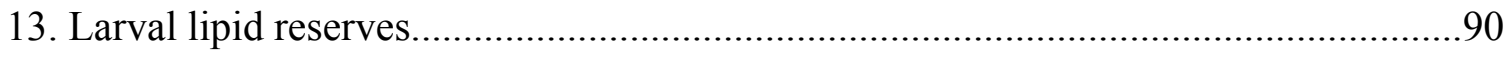

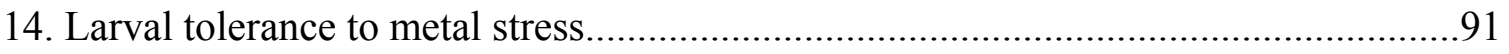

15. Larval development period in a metal environment...........................................92

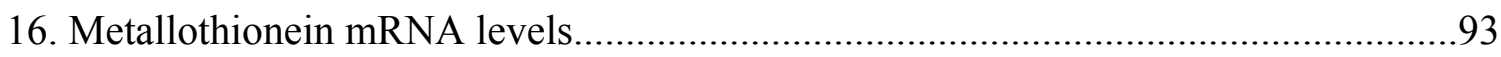

17. Extended quiescence reduced female adult body mass.........................................117

18. Extended quiescence increased starvation tolerance of adult females.....................119

19. Extended quiescence increased longevity of adult females.................................120

20. Extended quiescence altered reproductive strategy...........................................122 
21. Extended quiescence reduced tolerance to starvation of progeny first instar larvae. 123 


\section{LIST OF ABBREVIATIONS AND ACRONYMS}

AaMtn Aedes aegypti metallothionein

APS Aedes physiological saline ATP Adenosine triphosphate $\mathrm{Cu}^{2+}$ Copper $\mathrm{CuSO}_{4}$ Copper sulfate DDT. Dichlorodiphenyltrichloroethane DNA. Deoxyribonucleic acid ICP-MS inter-coupled plasma spectroscopy

JH. .Juvenile Hormone mRNA .Messenger ribonucleic acid NSL Neutral storage lipids

PAHO. .Pan American Health Organization PBS Phosphate buffered saline ppm .part per million qPCR. Quantitative polymerase chain reaction RNA .ribonucleic acid $\mathrm{rpL} 32$ Ribosomal protein subunit L32 RSW Reconstituted soft water

TAG. Triacylglycerol WHO. World Health Organization 


\section{Chapter 1: Introduction}

\subsection{Aedes aegypti: Global distribution and success as a pathogen vector}

Aedes (Stegomyia) aegypti (Linneaus) is the major urban vector of dengue viruses, yellow fever and Chikungunya worldwide. Aedes aegypti most likely originated in Africa and colonized other continents via trading and transport ships that resupplied in African ports during the fifteenth through seventeenth centuries (Christophers, 1960). $A$. aegypti probably was introduced to the rest of the world by eggs and larvae carried within freshwater reservoirs on trade ships. Historical records indicate that Aedes aegypti occupies regions between the northern January and southern July $10^{\circ} \mathrm{C}$ isotherms (Fig. 1). Given the occurrence of concurrent outbreaks of dengue in Asia, Africa and North America in the late $1700 \mathrm{~s}$, it is probable that Aedes aegypti, has had a wide a distribution throughout the world's tropics for at least the last two centuries.

The successful eradication of this mosquito in much of Central and South America by the 1970s was possible because of the large and coordinated yellow fever eradication effort initiated by the Pan American Health Organization (PAHO) in the 1950s and 1960s (Schliessman and Calheiro, 1974). However, as control efforts diminished throughout the 1970s, A. aegypti recolonized regions of both North and South America followed by epidemics of dengue and the emergence of dengue hemorrhagic fever (Gibbons and Vaughn, 2002). A. aegypti was common in southern Europe and North Africa (before World War II) (Kumm, 1931), but was eliminated by post war malaria eradication efforts and widespread use of DDT (Reiter, 2001). While A. aegypti currently exhibits a wide distribution in most tropical and subtropical areas, the historical 
records show a wider range, particularly in parts of Europe, North America and Australia. Overall, the geographical distribution of A. aegypti is dynamic and variable and population abundances vary greatly over time (Christophers, 1960). Global climate change with its increased temperature, humidity and rising sea levels will likely broaden this range (Gubler, 2004). The close association of A. aegypti with domestic environments provides this species microenvironments which are highly modified by humans such that they can avoid the effects of unpredictable weather (Higa, 2011). For example, $A$. aegypti's use of domestic water receptacles reduces its dependence on rainfall to provide larval habitats; in addition, the tendency for adults to rest indoors provides shelter and protection from external environmental conditions.

Although A. aegypti is characterized as a tropical mosquito, it has been shown to endure relatively adverse environmental conditions (Christophers, 1960). Field collected data indicates that $A$. aegypti exist and transmit dengue in desert townships where the mosquitoes avoid hot ambient temperatures $\left(>40^{\circ} \mathrm{C}\right)$ in summer by exploiting household containers like evaporative coolers and underground concrete water tanks (Russel and Webb, 2005). At the other temperature extreme, observations from the field have recorded viable A. aegypti larvae in ice cold water (Christophers, 1960). Although temperature affects larval developmental time, i.e., growth rate and survival rate, temperature alone does not determine larval abundance. Rainfall contributes significantly to larval abundance in areas where rain-filled containers are the primary larval sites. Aedes aegypti females readily oviposit in human-made containers holding water and are therefore not completely reliant on rainfall to provide the appropriate stimulus to hatch eggs. The highly anthropogenic nature of $A$. aegypti means that not only climate, but 
humans also drive the distribution of this species. The domestic characteristic of $A$. aegypti, along with the findings presented in this dissertation, may explain A. aegypti's wide geographic distribution along with its success as a vector. For example, vertical (transovarial) transmission of dengue viruses within eggs may retain the dengue viral pathogen during inter-epidemic periods or even from season to season thus preserving the virus reservoir within the mosquito population (Angel and Joshi, 2008). Despite the ability of A. aegypti to persist in urban environments resulting in dengue epidemics in many countries, population suppression or even eradication of this species is possible, however, it requires both coordinated efforts and a sustained commitment to vector management (Ballinger-Brown and Elder, 2009; Morrison et al., 2008).

\subsection{Dissertation justification and objective}

Over the last 25 years, there has been a global increase in both the distribution of A. aegypti and epidemic dengue virus activity (WHO, 2012; Jansen and Beebe, 2010 and references therein). In terms of morbidity and mortality, dengue and dengue hemorrhagic fever have been called the most important vector-borne viral diseases (WHO, 2012). Worldwide, there are approximately 2.5 billion people at risk of infection and there are 50 to 100 million cases per year (PAHO, 2012; WHO, 2012). About twothirds of the world's population lives in areas infested with dengue vectors, mainly $A$. aegypti and Aedes albopictus. All four dengue virus serotypes are known to affect urban populations (WHO, 2012). The dengue transmission cycle is heavily influenced by environmental conditions, human behavior and demographic changes. Climate change notwithstanding, uncontrolled population growth in urban areas of the tropics will likely 
increase the frequency of future dengue outbreaks along with an increase in the movement of both vector and virus reservoirs via modern transport and trade (Gubler, 2004; Jetten and Focks, 1997). In addition, the lack of effective mosquito management in developing countries will likely exacerbate future dengue outbreaks.

The $A$. aegypti vector is progressively adapting to living in cities with humans which poses significant challenges in terms of vector and disease control. In the absence of a vaccine, vector control strategies need to include a comprehensive assessment of the factors affecting the propensity of an area to harbor dengue vectors and viruses. Information on the integrated nature of human-mosquito-pathogen ecology is fundamental to the development of improved, perhaps even sustainable, vector control measures in urban environments. Current mosquito control strategies have failed because of the great potential of insects to develop insecticide resistance and the overall environmental threat posed by insecticides (Hemingway and Ranson, 2000; Hemingway, et al., 2004). To increase our knowledge of human-mosquito-pathogen ecology we need to have a better understanding of the physiology and life history traits of this pathogen vector.

It is the objective of this dissertation to describe the physiological ramifications of a previously uninvestigated life stage of the dengue vector $A$. aegypti. The knowledge acquired from this body of work can potentially mitigate urban pathogen transmission dynamics as it identifies and characterizes a vulnerable life stage of this mosquito, i.e., pharate first instar aseasonal quiescence, or more simply, "egg quiescence". With this information vector control agencies can make better informed decisions regarding control strategies. This dissertation is the first to report on the effects of egg quiescence in this 
vector mosquito and is coupled with a discussion of how the anticipatory plasticity that results from egg quiescence has contributed to the geographical distribution and success of A. aegypti as a pathogen vector. The research presented here provides evidence of anticipatory plasticity while also demonstrating that the life history trait responsible for A. aegypti's geographic success also makes this vector susceptible to pollution stress such that even low levels of environmentally relevant larval metal stress have wide and far reaching physiological consequences. These results suggest that $A$. aegypti exposed to urban metal pollution are likely at higher risk of increased vector capacity than their nonpollution stressed counterparts.

\subsection{Dissertation organization}

Chapter Two will review the literature pertinent to several underlying themes of the dissertation: dormancy in insects, phenotypic plasticity and the consequences of pollution stress. Chapters Three, Four and Five are reprints of articles previously published in peer reviewed journals that elucidate $A$. aegypti's metal tolerance physiology and its associated fitness costs and the previously uninvestigated physiological trade-offs of egg quiescence. Chapter Six examines the significance of these findings within the scope of urban vector ecology and the epidemiological consequences of uncontrolled urban expansion. 


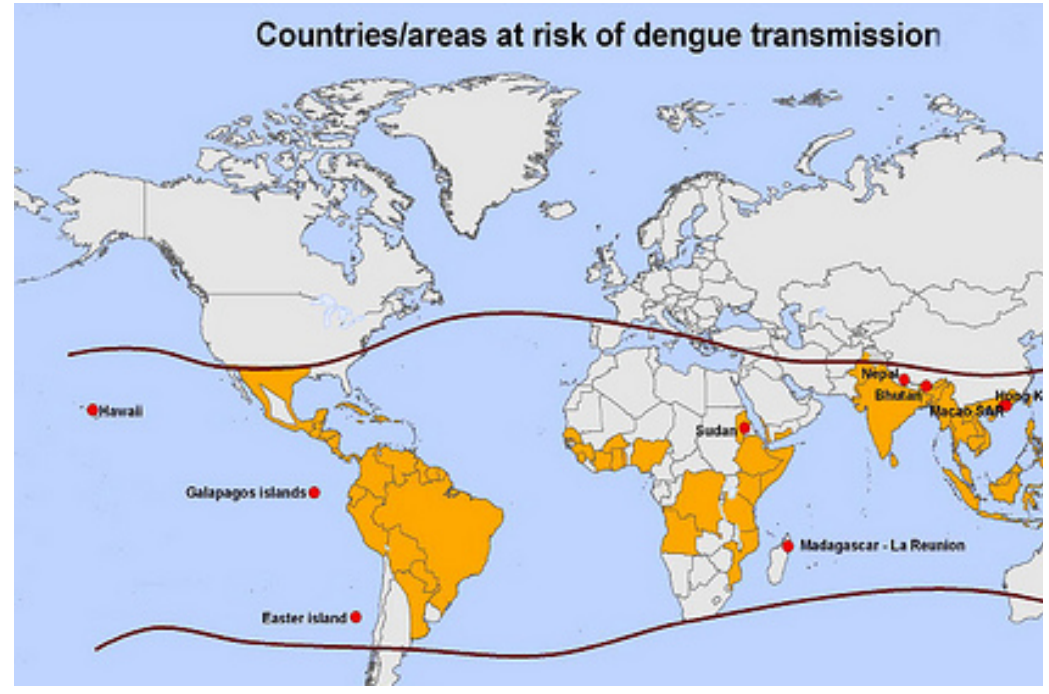

Fig. 1 Current global map dengue transmission risk (in yellow) (WHO 2012), also showing the hypothetical $10^{\circ} \mathrm{C}$ global winter isotherm distribution limits of $A$. aegypti proposed originally by Christophers in 1960 (Map courtesy of WHO 2012). 


\subsection{References}

Angel, B. and V. Joshi (2008). "Distribution and seasonality of vertically transmitted dengue viruses in Aedes mosquitoes in arid and semi-arid areas of Rajasthan, India." Journal of Vector BGorne Diseases 45(1): 56-59.

Ballenger-Browning, K. K. and J. P. Elder (2009). "Multi-modal Aedes aegypti mosquito reduction interventions and dengue fever prevention." Tropical Medicine \& International Health 14(12): 1542-1551.

Christophers, R., Ed. (1960). Aëdes aegypti (L.) the Yellow Fever Mosquito: its Life History, Bionomics and Structure, Cambridge University Press.

Gibbons, R. V. and D. W. Vaughn (2002). "Dengue: an escalating problem." BMJ 324(7353): 1563 .

Gubler, D. J. (2004). "The changing epidemiology of yellow fever and dengue, 1900 to 2003: full circle?" Comparative Immunology, Microbiology and Infectious Diseases 27(5): 319-330.

Gubler, D. J. (2004). "The changing epidemiology of yellow fever and dengue, 1900 to 2003: full circle?" Comparative Immunology, Microbiology and Infectious Diseases 27(5): 319-330.

Hemingway, J., Ranson, Hilary (2000 ). "Insecticide Resistance in Insect Vectors of Human Disease " Annu. Rev. Entomol. 45 371-391.

Hemingway, J., N. J. Hawkes, et al. (2004 ). "The molecular basis of insecticide resistance in mosquitoes." Insect Biochemistry and Molecular Biology 34 653665.

Higa, Y. (2011). "Dengue Vectors and their Spatial Distribution." Tropical Medicine and Health 39(4): 17-27.

Jansen, C. C. and N. W. Beebe (2010). "The dengue vector Aedes aegypti: what comes next." Microbes and Infection 12(4): 272-279.

Jetten, T. H. and D. A. Focks (1997). Potential Changes in the Distribution of Dengue Transmission under Climate Warming. 57: 285-297.

Kumm, H. W. (1931). "The Geographical Distribution of the Yellow Fever Vectors. A Compilation of Material recorded in the Literature, unpublished Communications and certain Collections made by the Author in Nigeria, West Africa." American Journal of Hygiene Monograph Series(12). 
Manrique-Saide, P., V. Uc, et al. (2012). "Storm Sewers as Larval Habitats for Aedes aegypti and Culex Spp. in a Neighborhood of Merida, Mexico." Journal of the American Mosquito Control Association 28(3): 255-257.

Morrison, A. C., E. Zielinski-Gutierrez, et al. (2008). "Defining Challenges and Proposing Solutions for Control of the Virus Vector Aedes aegypti." PLoS Med 5(3): 0362-0366.

PAHO (2012). "Dengue Fact Sheet." Pan American Health Organization 10(38).

Reiter, P. (2001). "Climate change and mosquito-borne disease." Environmental Health Perspectives 109(Suppl 1): 141.

Russell, R. C., C. E. Webb, et al. (2005). "Mark-release recapture study to measure dispersal of the mosquito Aedes aegypti in Cairns, Queensland, Australia." Medical and veterinary entomology 19 (4): 451-457.

Schliessmann, D. J. and L. B. Calheiros (1974). "A review of the status of yellow fever and Aedes aegypti eradication programs in the Americas." Mosquito News 34: 19.

Scott, T. W., G. G. Clark, et al. (1993). "Detection of Multiple Blood Feeding in Aedes aegypti (Diptera: Culicidae) During a Single Gonotrophic Cycle Using a Histologic Technique." Journal of Medical Entomology 30(1): 94-99.

Vinogradova, E., V. Alekseev, et al. (2007). Diapause in Aquatic Insects, with Emphasis on Mosquitoes

Diapause in Aquatic Invertebrates Theory and Human Use. H. J. Dumont and M. J. A. Werger, Springer Netherlands. 84: 83-113.

WHO (2012). "Dengue and dengue hemorrhagic fever." World Health Organization Monograph Series(Fact sheet $\left.N^{\circ} 117\right)$. 


\section{Chapter 2: Aedes aegypti: A combination of a distinct life history trait, plasticity \\ and vector biology}

\subsection{Diapause vs. quiescence in mosquitoes}

In most regions of the world, the physical and biological conditions favorable for insect growth, development and reproduction generally prevail only during particular seasons. Rainfall pattern is the defining characteristic of the tropical season. The wet/dry cycle elicits a periodic flush of vegetation, periodic back-flooding of rivers and the seasonal creation of small puddles and vernal pools that are suitable larval habitats for mosquitoes (Denlinger, 1986). The increase in plant growth stimulated by the rains provides a wealth of new food resources for many phytophagous insects and larger herbivores, which in turn, are a source of blood meals for mosquitoes. Therefore, to synchronize growth and reproduction with favorable times of the year and to enhance survival during unfavorable periods, many species of insects undergo some kind of dormancy. Tauber, et al., (1986) define diapause as,

"a neurohormonally mediated, dynamic state of low metabolic activity. Associated with this is a reduced morphogenesis, increased resistance to environmental extremes, and altered or reduced behavior activity. Diapause occurs during genetically determined stage(s) of metamorphosis, and its full expression develops in a speciesspecific manner, usually in response to a number of environmental stimuli that precede unfavorable conditions. Once diapause has begun, metabolic activity is suppressed even if conditions favorable for development prevail."

David Denlinger, Hugh Danks, as well as other authorities on the subject, follow similar criteria for defining diapause. In short, diapause is a genetically pre-programmed regime that once initiated needs to proceed to its genetically predetermined endpoint. Diapause can occur in any stage of mosquito development, however, only one diapausing 
stage is typical of most mosquito species, although a few may overwinter in two different developmental stages, but in different regions of their geographic distribution (Vinogradova, 2007).

Quiescence, on the other hand, is an immediate response to environmental stress that can occur at any time during the lifecycle of an insect that is also characterized by decreased metabolism, activity and feeding, but it is terminated immediately by the return of favorable stimuli (e.g., temperature, flooding etc.) (Hahn and Denlinger, 2007). In contrast to diapause (a predetermined genetic program), quiescence is more accurately characterized as a physiological response to environment cues.

Egg or embryonic diapause, terms used quite often, occurs in mosquitoes of the genera Ochlerotatus, Aedes, and Psorophora (Vinogradova, 2007 and references therein). Neither term is entirely accurate because diapause in all of these cases occurs in the stage of the pharate first instar larva (embryonic development has been completed but the fully formed first instar larva remains within the chorion of the egg). This is the stage of diapause for $O$. dorsalis, $O$. nigromaculis, $O$. squamiger , O. hexodontus, A. cinereus, $O$. flavescens, O. triseriatus, and $O$. togoi, and is most likely true for other species as well. Egg diapause is manifested as a long stable arrest of hatching even when environmental conditions are favorable for this process. Diapause is terminated as a result of the reactivation of development by preprogrammed genetic and hormonal factors (Denlinger, 1986) that promote successful survival of winter (winter diapause) or summer (aestivation) (Vinogradova, 2007). The winter egg diapause is typical for mosquito species occurring in the temperate zone. In addition to egg diapause in mosquitoes, there is aseasonal quiescence, a state of inactivity induced by unfavorable environmental 
conditions and which ceases shortly after exposure to adequate hatching stimuli. Aseasonal quiescence is an adaptation to the peculiar conditions of special larval habitats, such as tree and rock holes, or transient ground pools, where water level may be subjected to large and abrupt fluctuation (Vinogradova, 2007). Such aseasonal quiescence results in an asynchronous hatching of eggs. Usually the first flooding induces hatching of the majority of the eggs, whereas the remainder of the eggs may hatch much later after a subsequent flooding episode. Asynchronous hatching determined by intrapopulation variation in sensitivity of individuals to environmental cues. Such arrest of hatching is a very useful adaptation favoring the conservation of populations after the early-spring freezing or after quick drying of water bodies - essentially a form of hedge-betting. Though egg diapause is quite distinct from aseasonal quiescence, in some cases it is very difficult to distinguish these phenomena. Egg diapause may be obligate or facultative. Obligate diapause is common in monovoltine mosquito species such as $O$. canadensis, $O$. hexodontus, O. squamiger, O. excrucians, and O. communis. It occurs spontaneously in each generation irrespective of environmental conditions and lasts usually for a long period, up to one year, from the end of spring/beginning of summer to the spring of the following year. On the other hand, facultative diapause is found in multivoltine species (e.g., A. vexans, O. caspius, A. cinereus) (Clements, 1992). This type of diapause is controlled by environmental conditions, mainly photoperiod and temperature. Temperatures changes are usually responsible for diapause termination in both monovoltine and multivoltine species.

Practically all eggs of Ochlerotatus, Aedes, and other genera require a hatching stimulus such as a change in temperature, relative humidity, repeated flooding and 
drying, mechanical stimuli and especially a decrease in concentration of dissolved oxygen in water in addition to submergence in water (Clements, 1992). The efficiency of these various stimuli depends on the physiological state of the diapausing eggs. It has frequently been shown that eggs respond more readily to a hatching stimulus if they have previously been held at a high humidity. It has been well documented that egg viability decreases with increased duration of quiescence, the reason for which has remained unclear (Clements, 1992) and is the subject of chapter four.

\subsection{Anticipatory phenotypic plasticity - environment, development and genes}

The ability of mosquito eggs to survive long periods of drying is associated with species that inhabit transient water bodies. Accordingly, this dissertation demonstrates that the egg quiescence period of $A$. aegypti results in anticipatory phenotypic plasticity. A thorough literature search of the topic of alternative phenotypes in insects yields only one case describing plasticity in wild populations of mosquitoes (O'Meara and Lounibus, 1981). This is not to say that other cases do not occur in msoquitoes, but rather that an overwhelming majority of the work performed on phenotypic plasticity has been performed with insects other than mosquitoes (e.g., butterflies and crickets) (Fig. 1).

\subsubsection{Background and nomenclature}

As with the discussion of dormancy, any discussion of phenotypic plasticity in insects requires the clarification of some ambiguous and/or overlapping terms. The majority of this dissertation follows the conventions of Mary Jane West-Eberhard (WestEberhard, 2003) unless otherwise specified. Phenotypic plasticity is the ability of a single 
genotype to produce more than one alternative form of morphology, physiological state, and/or behavior in response to environmental conditions. Phenotypic plasticity, once shunned as an experimental nuisance, is today theorized to account for much diversity and possibly result in sympatric speciation (Moran, 1992; reviewed in West-Eberhard, 1989, 2003).

The term "phenotype" includes all aspects of an organism other than the genotype, from the enzyme products of the genes to learned behaviors and the effects of disease. Alternative phenotypes refer to two or more forms of behavior, physiological response, or structure maintained in the same life stage in a single population and not simultaneously expressed in the same individual. They are taxonomically widespread and occur in every life stage including sperm and eggs. Polyphenism refers to the existence of environmentally cued alternative phenotypes in a population, some polyphenisms arise when reaction norms (responses to the environment) become discontinuous so that the phenotype no longer changes gradually with graded changes in the environment (Simpson, et al., 2011). Polymorphism refers to the existence of morphologically distinct alternatives in a population. Alternative adaptations are different adaptive phenotypes maintained in the same life stage and the same population, but not necessarily simultaneously expressed in the same individual and represent contrasting character sets produced by the same genome, in effect allowing a single species to occupy more than one sympatric niche (West-Eberhart, 1989). Seasonal generations of multivoltine species (i.e., the species with more than one generation per year) often differ in their morphology, behavior and life history. This type of phenotypic plasticity, called seasonal polyphenism, is common among short lived organisms like insects and other arthropods, and is 
particularly well documented in butterflies and moths (reviewed in Shapiro, 1976; Tauber, et al., 1986; Brakefield, 1996; Brakefield et al., 2009). For example, different seasonal generations of lepidopterans and other insect orders may differ in morphology, color and patterning, body size, growth rate and duration of the larval period, longevity and reproductive traits (Fig. 1)

Phenotypic plasticity can be either responsive or anticipatory. Responsive plasticity is the result of the immediate effect of environmental factors on a population. Often the effect of nutrition (food quality and quantity) and temperature on life history traits often serve as examples of responsive plasticity. In contrast, anticipatory plasticity is induced in response to cues which signal future environmental conditions prior to the actual onset of these conditions (Esperk, et. al., 2012). Responsive plasticity is not necessarily adaptive i.e., selected and/or maintained by natural selection; however, anticipatory plasticity is usually thought to be so, although the anticipatory nature of a plastic response only provides circumstantial evidence of its adaptive basis.

Seasonal polyphenisms can typically be classified as anticipatory plasticity and are generally assumed to be adaptive responses to seasonally differing selection pressures (Shapiro, 1976; Nijhout, 1999; Brakefield et al., 2009). In the most common example color polyphenisms in lepidopterans - the adaptive nature of such polyphenisms has explicitly been shown. However, seasonal changes in organismal traits can also result from responsive plasticity as factors typically causing such changes also frequently show clear patterns of seasonal variation. Moreover, as seasonal polyphenisms usually are multitrait responses, it may well be the case that some traits show anticipatory plasticity while differences in others may be a result of responsive plasticity to environmental 
conditions. Nevertheless, attempts to explicitly confront these different explanations are limited (Esperk, et al., 2012). Some seasonal polyphenisms are tightly coupled with diapause. Individuals that enter diapause develop one adult phenotype, whereas those that do not develop a different phenotype (Denlinger, 1985; Tauber, et al., 1986; Nijhout, 1994). The seasonal polyphenism of the wing pattern of butterflies such as A. levana and Papilio xuthus (Japanese swallowtail), for instance, is closely associated with diapause (Endo, et al., 1985; Koch and Buckmann, 1987) while alternative seasonal wing pattern phenotypes of Precis coenia, Precis caureum, Pieris protodice, and Bicyclus species develop without diapause (Shapiro, 1976; Endo, et al., 1985; Smith, 1991).

Another possible manifestation of phenotypic plasticity is the reaction norm or norm of reaction. A reaction norm describes the range of phenotypic expression of a single genotype across a range of environments. In other words, an organism produces a phenotype that varies as a continuous function of the environmental signal (Stearns, 1989). A simple example would be the continuous increasing adult body size of an insect as a function of continuous increasing larval diet quantity and quality. There are three different mechanisms by which developmental processes can lead to reaction norms (Nijhout, 1999). First, an environmental variable (e.g., temperature) can have a direct effect on the rate or timing of biochemical processes (e.g., enzymatic activities) so that they no longer proceed at the same relative rates. The altered developmental processes then result directly in an altered phenotype. A possible organismal level example of this mechanism is the temperature dependent reaction norm of the color pattern of Bicyclus butterflies. However, color pattern variation in these butterflies is actually better correlated with overall development time than with temperature, which suggests that any 
mechanism that slows the rate of development will produce the same alteration of the color patterns as when development is slowed by lowered temperatures (Roskam and Brakefield, 1996). In a second mechanism by which developmental processes can result in reaction norms, the environmental variable leads to a change in the level of expression of certain genes, so that they are transcribed differentially in some environments than in others. The altered quantity of their gene products, which are required for normal development, then causes a change in phenotype. In a third mechanism, the environmental variable can lead to the expression of new genes, so that different patterns of gene expression and therefore different developmental programs run under different environmental conditions. The first two of these mechanisms can be categorized as allelic sensitivity, the environmental effect is direct and affects some aspect of development quantitatively. In contrast, the developmental switch produced by the third mechanism has been called regulatory plasticity (Schlichting and Pigliucci, 1995). In this case, the environmental effect is indirect and alters development qualitatively by changing which genes are expressed. All three mechanisms eventually result in phenotypic differences that may be perceived as qualitative; therefore, observing the phenotype alone cannot reveal what mechanism was at work. Moreover, each of the alternative developmental pathways produced by regulatory plasticity can, in turn, exhibit allelic sensitivity. The environment can therefore have a hierarchical effect on development, with one environmental variable producing two or more phenotypic variants that each respond differently to a second environmental variable.

Some polyphenisms arise when reaction norms become discontinuous so that the phenotype no longer changes gradually in correlation with graded changes in the 
environment. A polyphenism can be thought of as a reaction norm with a threshold, such that a discrete phenotypic switch is tripped when a critical range of environmental variation is reached. In polyphenic development, the environmental cue is often a substitute stimulus for the environmental variable to which the polyphenism is an adaptation. Photoperiod can serve as a substitute stimulus to predict the onset of winter resulting in a phenotypic form that is cold adapted. The sensitive period during which this stimulus is effective is often far removed from the time at which the affected phenotypic characters develop. It is therefore unlikely that, in anticipatory polyphenic development, the environmental variable has a direct effect on development via allelic sensitivity. The environmental effect is indirect and acts by changing the patterns of hormone secretion.

It is also possible for polyphenic development to occur in the presence of a perfectly smooth reaction norm, if the organism occurs in an environment that varies abruptly or discretely so that different generations develop under different environmental conditions. Some seasonal polyphenisms of insects can be of this kind, particularly in species that have two or three generations per year, so that each generation is likely to develop during a different season. When such organisms are exposed to intermediate environmental conditions, they develop intermediate phenotypes. Thus, phenotypic plasticity that, in nature, would typically be expressed as a polyphenism can be expressed as a reaction norm under controlled or artificial conditions and is an issue addressed in chapter five (Roskam and Brakefield, 1996). 


\subsubsection{Polyphenic development in insects}

Polyphenic development is controlled at the environmental, developmental and genetic levels and regulated at the transcriptional, translational and metabolomic levels (reviewed by Nijhout, 1999 and $\mathrm{Wu}$, et al., 2003). This present review, however, will focus mostly on the effects of environment and development on phenotypic plasticity rather than the effects of genetics as it is the objective of this dissertation to demonstrate that environment and development are the determining factors of anticipatory plasticity in A. aegypti.

Effect of Environment - Environmental conditions affect phenotypic development (West- Eberhard, 1989; Moran, 1992). Insects use a variety of environmental factors as cues for polyphenic development such as photoperiod and temperature, crowding, pheromones and changes in food quality. For example, the gaudy commodore butterfly (Precis octavia), the common bush brown butterfly (Bicyclus safitza) and the squinting bush brown butterfly (Bicyclus anynana) use temperature and the map butterfly (Araschnia levana) uses photoperiod as a cue to determine alternative phenotypes of adults (Nijhout, 1999 and references therein). Sometimes, both these environmental factors induce polyphenic development and can substitute for one another as is the case with the common buckeye butterfly (Precis coenia). Crowding controls the wing length polyphenism of the field cricket Gryllus rubens. Crowding, photoperiod, and temperature all control the complex alate - apterous polyphenisms in aphids, and food quality and pheromones control caste polyphenism in the ant Pheidole bicarinata. In many insects there is a well-defined period during embryonic or larval development when the 
individual is sensitive to environmental cues. The environment sensitive period that precedes the development into the final adult phenotype may be as brief as a few days or as long as several months (refer to Fig. 2 throughout remainder of discussion of plasticity). For example, the environment sensitive period for the seasonal wing pattern polyphenism of the butterflies, Precis coenia, Araschnia levana, Polygonia caureum, Bicyclus safitza, and Bicyclus anynana is during the mid to later larval instars but not the pupal stage (Nijhout, 1999). The pupal color polyphenisms of the cabbage white butterfly (Pieris brassicae), and the zebra swallowtail (Eurytides marcellus) are controlled during a brief light sensitive period shortly before pupation. In contrast, the pupal color polyphenisms of the black swallowtail, (Papilio polyxenes) and the spicebush swallowtail (Papilio troilus) are determined during the last larval instar. In aphids, alate - apterous polyphenisms are controlled either during the larval stages or during the embryonic stage, depending on the species. Environment sensitive stages of aphids occur while the developing eggs are still within the genital tract of the mother. The embryos do not appear to sense the environmental cues directly, but are cued through the mother, usually by means of hormones (Hardee and Lees, 1983). In Gryllus rubens, the wing length polyphenism is controlled by crowding during the last half of larval life and the effects of crowding are reversible as late as the last larval instar. The lag time between the environmental signal and the development of the alternative phenotype may be the result of the time certain relevant developmental events take to complete or it may arise because the signal causes a reprogramming of developmental events that will take place at some time in the future. The latter scenario most likely is responsible for $A$. aegypti's anticipatory plasticity, a central hypothesis of this dissertation. 
Some Lepidopteran larvae developing early in the season produce larger adults than do late season larvae. On the other hand, in some other species, the generation with late season larvae attains larger size. Hypotheses for this seasonal difference include the selective pressures of higher predation in spring and higher overwintering survival of larger individuals. However, these adaptive explanations explain only when the late season larvae grow larger than the early season larvae. In cases where the insects developing early in the season grow larger, size differences between generations have been ascribed to responsive plasticity, i.e., the immediate effects of environmental factors. Host plant quality has been shown to decline towards the end of the season. Individuals with their larval period in spring are therefore expected to achieve larger adult sizes for this reason alone. Nevertheless, there are no studies explicitly exploring the effect of food quality as a mediator of inter-generation size differences. Likewise, another candidate for the responsive basis of the inter-generation size differences relates to the tendency of ectotherms to grow slower but to mature at larger size when exposed to lower temperatures during the juvenile period. The generation experiencing lower temperatures during the larval life could thus attain a larger adult size also in response to temperature. (Esperk, et al., 2012). In addition, body size difference are not the only parameter to consider since nutritional reserves are not necessarily proportional to body size as demonstrated in chapter five. Regardless, larval environment clearly has a powerful effect on adult phenotypes.

Developmental Effects - In polyphenic development, environmental factors act by altering some aspect of development in an orderly and predictable way (West-Eberhard, 2003). Sometimes this effect is direct, such as when a change in temperature alters the 
rate of a critical developmental process. Often, however, the environmental effect is indirect triggering hormones to reprogram future developmental sequences.

Hormonal control of developmental switches - The developmental control mechanisms that cause polyphenic development have been studied in a number of insects. The mechanism appears to involve a developmental switch that is controlled by one or more hormones (for a complete discussion of insect hormones see Nijhout, 1994). Hormonal control of polyphenic development is so widespread that all insect polyphenisms are likely to be controlled by changes in endocrine physiology. Two hormones, juvenile hormone and ecdysone are most commonly involved in polyphenic development; in addition, neurosecretory hormones also play a critical role in aphids, locusts, and some butterflies (Nijhout, 1994).

Polyphenisms can evolve if one or more of these components of an endocrine switching mechanism become sensitive to environmental variables. Regulation of polyphenic development by means of changes in the concentration of hormones appears to be quite common. Regulation by means of fluctuating hormone concentration is best known from studies on the control of metabolism and homeostasis, although hormonal fluctuations also accompany many developmental events, such as molting and metamorphosis. There is, however, an important difference between the role of hormones in physiological regulation and their role in developmental regulation, namely, that in physiological regulation the response to a hormone is often proportional to its concentration, whereas in development the response is typically all-or-none (Nijhout, 1994). One functional explanation for this difference is that development is progressive; specific developmental stages at which decisions must be made typically occur only 
once, and only for a brief period of time. In development, hormones trigger switches that alter the pattern of gene expression and either initiates certain developmental events or direct development into specific alternative pathways. As hormone levels rise and fall, the developmental response changes qualitatively, rather than quantitatively. It is possible for organisms to show a graded developmental and phenotypic response to graded changes in hormone concentration, but the mechanism of graded change appears to be quite different from that involved in homeostatic regulation. A graded developmental response can come about when different tissues of an organism or different features of a tissue either have different response thresholds to a hormone or require different lengths of exposure to a hormone to mount a normal response. Such intermediate developmental responses are the result of the additive effects of two or more types of cells, tissues or systems within an organism (Riddiford, 1996). Similarly, graded changes in the timing of hormone secretion can cause a graded phenotypic response when a hormone arrests an ongoing developmental process at different times. There are many instances in which artificial elevation of a hormone titer appears to be sufficient to cause a polyphenic switch. For instance, implantation of active corpora allata (the gland that secrete juvenile hormone) into larvae of the migratory locust, Locusta migratoria, that were crowded and would normally have developed into the melanic gregarious morph caused them to develop the green pigmentation characteristic of the solitary morph (Staal, 1961). An injection of 20-hydroxyecdysone early in the pupal stage can cause Precis coenia that would otherwise have developed into an adult of the autumn morph to develop the summer phenotype instead (Rountree and Nijhout, 1995). Exogenous juvenile hormone also induces queen development in the honey bee Apis mellifera and soldier development 
in various ants and termites (Wheeler and Nijhout, 1984). In crickets, there is a tight correlation between the levels of juvenile hormone esterase and wing length polyphenism (Zera and Tiebel, 1989, Fairbairn and Yadlowski, 1997, Zera and Denno, 1997). In addition, it has been demonstrated the juvenile hormone serves as the endocrine basis for reproductive life history tradeoffs in A. aegypti (Clifton and Noriega, 2011; 2012). More recently, Ishikawa et al. (2013) demonstrated that juvenile hormone may play an important role in the differentiation of the wing-morph polyphenism of the vetch aphid Megoura crassicauda .

Changing the thresholds of sensitivity to a hormone in the presence of a constant concentration of hormone is equivalent to changing hormone concentration in the presence of a constant threshold of sensitivity. Some polyphenic systems, such as the soldier - worker polyphenism of the ant genus Pheidole are controlled, in part, by changes in the threshold of sensitivity to juvenile hormone (Goodman and Cusson, 2012; Nijhout, 1994, 1999). Although the details of how a threshold of sensitivity to a hormone is achieved physiologically are not entirely known, it is known that in at least some systems sensitivity is proportional to the amount of hormone receptor in the target tissue. Binding affinity of the hormone-receptor complex and variation in the intracellular response mechanism also play roles in determining the apparent threshold of sensitivity. In addition, sometimes different splice variants or phosphorylated states of a receptor have different binding affinities; other times hormones are inactivated by binding proteins which must be saturated before residual hormone is free to bind the receptor (Alberts, et al., 2002). 
Hormone-sensitive periods - When hormones act as developmental switches, the timing of their secretion is as important as their concentration. There are critical hormone-sensitive periods, just as there are critical environment-sensitive periods (Nijhout, 1994, 1999). Elevation or depression of hormone concentration outside of the critical period for a given tissue or response is irrelevant and without effect. The general explanation for the existence of hormone sensitive periods is simply that development is progressive and irreversible, so that decision points - that is, times during which a developmental trajectory can be altered - are reached only once, and the progression of development beyond that point makes the decision irrevocable. The timing of hormonesensitive periods is thus determined by the attainment of certain stages in the developmental process. The actual mechanism responsible for the appearance of hormone sensitive periods appears to be related to the behavior of hormone receptors in the cells that are the targets of the hormone. In many instances, tissues have been found to be responsive to hormones only at or very near the time at which the hormone is normally secreted (Nijhout, 1994). This phenomenon is believed to be regulated at the level of the hormone receptor (Riddiford, 1996), a notion that is supported by enormous temporal and spatial variation in hormone receptor expression during development (Dufty, et al., 2002).

Developmental and physiological studies have revealed several independent mechanisms that regulate polyphenic development in insects. All insect polyphenisms, exhibit environment sensitive periods during which some kind of external stimulus alters the developmental fate of the animal. Each environmental signal appears to have its effect on development by reprogramming the hormone response mechanism. This 
reprogramming can be the result of changes in one or more of five very different mechanisms (Nijhout, 1999):

a) the titer of a hormone during a hormone-sensitive period,

b) the threshold of sensitivity to a hormone,

c) the timing of hormone secretion,

d) the timing of a hormone-sensitive period, or

e) the cellular response to the hormone.

Each of the five mechanisms that control polyphenic switching can itself be controlled through several distinct sub-mechanisms or pathways. For instance, the concentration of circulating hormone can be changed by altering its rate of synthesis, its rate of secretion, or its rate of degradation by catabolic enzymes. The titer of juvenile hormone, for instance, is regulated independently by both synthesis and degradation mechanisms (Goodman and Cusson, 2012). The various mechanisms and pathways for developmental reprogramming each use different gene products and require a different regulatory system. Each mechanism is presumably under independent physiological and genetic control and can therefore vary and evolve independently of the others. It is important to recognize that these five mechanisms are present as part of the normal "monophenic" developmental system of insects. They do not need to evolve or be maintained separately just to serve polyphenic development. All that is required for the evolution of a polyphenism is that one or more of these mechanisms becomes physiologically linked to a process that is sensitive to an environmental variable such as temperature, photoperiod, nutrition, crowding, or pheromones. The developmental system of insects is therefore pre-adapted in many ways for the rapid evolution of 
phenotypic plasticity. A switch like polyphenic control of development is relatively easy to establish because the temporal relationship between a hormone peak and a hormonesensitive period is easily manipulated, either by an environmental signal or by mutation of one of the many components of the pathway. Even a quantitatively small relative shift of hormone peak and hormone-sensitive period can bring them completely out of register, thus causing a qualitative switch in development.

Genetic Effects - Although the effect of genetic variation on phenotypic plasticity is not relevant to this dissertation, a brief discussion of genetic effects follows as no discussion regarding plasticity would be complete without mention of them. The prevalence of phenotypic plasticity and reaction norms across a wide variety of taxa suggests that the evolutionary change of phenotypes can be initiated either by a change in environment or by a genetic mutation (West-Eberhard 1986, 1989, 2003; Schlichting and Pigliucci 1998). Once initiated, the new phenotype can be further altered and adaptively refined and stabilized by further changes in the processes that give rise to phenotypes. Among the targets of this change are the genes whose products participate in the various endocrine processes described above.

Evolution of polyphenic development can occur through changes in the environment sensing mechanisms or through changes at any level in the developmental switching mechanism. Such evolutionary change can take many forms, from a simple alteration in the threshold for the response to a changing environmental signal, to the evolution of new physiological or morphological adaptations of one or both alternative morphs. Evolution depends, in part, on the availability of the appropriate genetic variation; many aspects of a polyphenic response mechanism are variable in ways that 
can lead to adaptive evolution of the polyphenism. Genetic variation in the mechanisms that monitor and integrate environment signals is well documented (Stearns, 1989; WestEberhard, 2003; Simpson et al., 2011). Genetic variation in the environment sensing mechanism is often expressed as variation in the ability to switch between alternative developmental pathways under a constant environmental signal. (Tauber et al., 1986; for a complete discussion see West-Eberhardt, 2003).

Genetic variation in the endocrine control mechanism is also known to occur, although little is known about the causes of this variation. Although variable juvenile hormone metabolism is responsible for variation in the expression of wing length polyphenism in various species of Gryllus crickets (Zera, et al., 1989, Zera and Zhang 1995), the genetic control of wing morphology is mediated through a juvenile hormone mediated switching system (Zera, 2006). Genetic variation of the developmental response downstream of the endocrine signal is also possible. Indirect evidence about genetic variation at the mechanistic level comes from quantitative genetic studies of plasticity in both the plant Phlox and the fruit fly Drosophila. For instance, if there is a solely genetic control of plastic character and of the plasticity of that character the plasticity of that character can be quantified by the difference in the mean values of the character of siblings raised in different environments (Scheiner and Lyman 1991; Shirley and Sibly, 1999). These findings can be extended to polyphenic traits, and if polyphenisms evolve from continuously plastic traits by the addition of a hormone mediated switching mechanism, then it is likely that the mean value of a character and the degree of differentiation of a character in alternative phenotypes are also controlled independently. Thus, once a polyphenism is established, each of the alternative morphs can undergo 
further adaptive evolution by changes in the developmental response system downstream of the endocrine signal (Moran, 1992; Simpson, et al., 2011; West-Eberhard, 2003).

\subsubsection{Alternative phenotypes in mosquitoes}

The best known example of phenotypic plasticity in wild populations of mosquitoes occurs in Pitcher-plant mosquitos (Wyeomyia smithii Coq.) where the ratio of two alternative reproductive patterns varies along a South-North cline, ending in fixation of a single physiological and behavioral phenotype in the northern United States (O'Meara and Lounibus, 1981). In the south adult females emerge with undeveloped ovaries and show both alternatives: some are entirely anautogenic and others mature their first batch of eggs autogenously using resources derived from larval feeding and adult nectar feeding (reviewed in Foster, 1995). However, if southern $\left(30^{0} \mathrm{~N}\right.$. latitude) larvae are given an artificially superior diet, nearly all (99\%) resulting females produce a clutch of eggs without a blood meal, showing that autogeny is facultative. A South-to-North cline of increasing frequency of autogeny corresponds to a cline of decreasing larval densities within pitcher plants with blood feeding completely absent North of $40^{\circ} \mathrm{N}$. latitude even if females are reared on an inferior larval diet. There has thus been an evolutionary change permanently fixing the autogenous phenotype in northern populations. Furthermore, obligatorily autogenous northern females emerge with precocious ovarian development and mate earlier than southern (Florida) females (O’Meara and Lounibus, 1981). The northern populations thus appear to show increased specialization to autogeny associated with fixation of the autogenous phenotype. In species and genera of mosquitos with obligate autogeny, females often have even more 
extreme autogenous specializations, such as modified mouthparts unable to pierce vertebrate skin.

Under laboratory conditions, however, researchers have reared 'small' and 'large' phenotype adult mosquitoes (along with intermediate phenotypes) through larval diet manipulations, this clearly being an example of responsive plasticity resulting in a reaction norm (Briegel, 1990, Briegel et al., 2002; Caroci, et al., 2004).

The research presented within this dissertation provides evidence that the duration of the aseasonal quiescence period of $A$. aegypti eggs, i.e., the pharate first instar stage, serves as an environmental cue signaling the end of a dry period. It is likely that subsequent developmental switching in early stage larvae results in an adult phenotype best suited for the environmental conditions that follow. There is, however, a trade-off larvae hatched from long duration quiescence eggs are less tolerant of stress, while their subsequent adults are more tolerant of it. The trade-off of stress tolerance between larval and adult stages is the subject of chapters four and five. Whether this trade-off is an alternate polyphenism or a reaction norm remains to be determined. More importantly, this trade-off has epidemiological ramifications. Aedes aegypti's vector capacity is affected by larval stress (Alto and Lounibous, 2012). In chapters four and five extended pharate first instar quiescence is shown to be a form of larval stress.

\subsection{Metal pollution stress during the larval stage}

The presence of the yellow fever mosquito larvae has been confirmed in polluted habitats such as drains containing domestic wastewater and in man-made aquatic habitats (Barrera, et al., 2006, 2008; Chinery, 1995), habitats previously not associated with this 
species of mosquito. These observations suggest that pollutant tolerant strains of Aedes species of mosquitoes are developing. Aedes aegypti has, in fact, adapted to urban environments; domestic and feral forms have been recognized in Africa (Crovello and Hacker, 1972). It is possible that the urbanization process can enhance the survival of pathogen vectors by providing suitable habitats and subsequently increasing the probability of the transmission of pathogens in high-density urban environments (Frankie and Ehler, 1978). In urban environments there are many sources of aquatic metal pollution from run off, fall out, indiscriminant use of metal containing herbicides etc. However, larval breeding containers are of particular interest as many are made of metal, i.e., septic tanks, discarded metal cans, cisterns, drains, sewers and dumpsters. Therefore, metal tolerance needs to be specially considered with respect to the adaptations this disease vector is undergoing (Ocampo and Wesson, 2004; O'Meara et al., 1992; Posthuma and Van Straalen, 1993; Poupardin, et al., 2008). Adaptations to novel environments provide distinct advantages to the populations undergoing these adaptations; however, adaptations have fitness costs. These fitness costs, in turn, will affect the disease transmission dynamics of a vector i.e., vector capacity (Alto, 2005, 2008).

Metal tolerance is the physiological adaptation of an individual over the course of its lifetime (responsive plasticity). Individuals of a given population can tolerate increased concentrations of ambient metal as a result of the up regulation of detoxification, sequestration and excretion mechanisms and the down regulation of uptake mechanisms. The yellow fever Mosquito, being a container breeder, is subject to greater selection pressure of metals in the environment than other species of mosquitoes 
that transmit disease. To date, little is known about the physiological cost of adaptation to metal pollution or how this might affect the fitness of the mosquito population and its ability to transmit diseases (Sarkar et al.,2004).

Of the many metals that $A$. aegypti are exposed to in an urban environment, copper is of primary interest. Copper is an essential trace element with an important role in several cellular processes such as respiration, oxidative stress defense, and immune function (Puig and Theile, 2002). Copper is found in a number of important enzymes and proteins, such as, cytochrome $c$ oxidase, $\mathrm{Cu}$-Zn-superoxide dismutase 1 (SOD1), tyrosinase, lysyl oxidase and multicopper oxidase (Egli, et al., 2006a; Gaetke and Chow, 2003). Copper is also used indiscriminately as an algaecide, fungicide, root treatment agent and in the manufacture of artificial containers (Barrera et al., 2008; Mireji et al., 2008). Aedes aegypti mosquitoes need to protect themselves from toxic levels of this agent while at the same time preserving levels essential to basal metabolism. Copper metabolism, toxicity and tolerance can also serve as a model for the same processes involving mercury, silver, zinc, and cadmium as each of these transition metals share a similar detoxification pathway via metallothionein binding (Hare, 1992).

Metallothionein - Metallothioneins are small, ubiquitous, cysteine rich proteins with a high affinity for transition metals (Amiard, et al., 2006; Egli, et al., 2006b; Kägi, 1991). They are found in many taxa, ranging from yeast to humans. Metallothioneins have an important role in the homeostasis of essential metals, the detoxification of nonessential metals and the oxidative stress response. They exert their protective effect by binding and sequestering metal ions, thus keeping the concentration of free metal ions relatively low while simultaneously providing a reservoir of essential metals for 
metalloprotein synthesis and the various other roles metals play in metabolism.

Metallothioneins also provide antioxidant protective properties. Antioxidant response element (ARE) promoter sequences of metallothionein genes have been shown to participate in the oxidative stress regulation of gene transcription in a wide variety of taxa (Andrews, 2000). The conservation and the presence of multiple gene copies of metallothionein imply an important cellular function, i.e., metal homeostasis and detoxification of metals (Egli, et al., 2006b; Maroni et al., 1987). To date no A. aegypti metallothioneins gene sequences have been annotated in the $A$. aegypti genome (Nene, et $a l ., 2007)$ however, the metallothionein gene is evidently present in A. aegypti and is the subject of chapter three. A literature search of metal tolerance physiology in mosquitoes yields only five or six papers published on the subject. Clearly, there exists a need for a better understanding of the mechanisms of metal toxicity and tolerance in this important pathogen vector.

\subsubsection{Vectorial consequences of larvae metal stress}

Most studies on vector competence have focused on the environmental conditions experienced by adults, but the larval environment shapes the adult phenotypes of mosquitoes and may, thereby, alter vector competence for arthropod-borne viruses (Alto, et al., 2008; Smith, et al., 2009). Mosquito vector competence studies have demonstrated that larval environmental conditions modify the susceptibility to infection and transmission of pathogens (reviewed in Alto and Lounibous, 2013). Studies on the effects

of nutrition, competition, temperature and insecticides during the larval stages on adult vector competence for arboviruses have shown that the larval environment may 
significantly increase susceptibility to infection, dissemination and virus transmission. These effects show multiple and environmentally specific effects on the barriers to virus infection. Although no previous studies examined larval metal stress specifically, metal stress probably alters vector competence, and an investigation of which, is the subject of chapter three. 


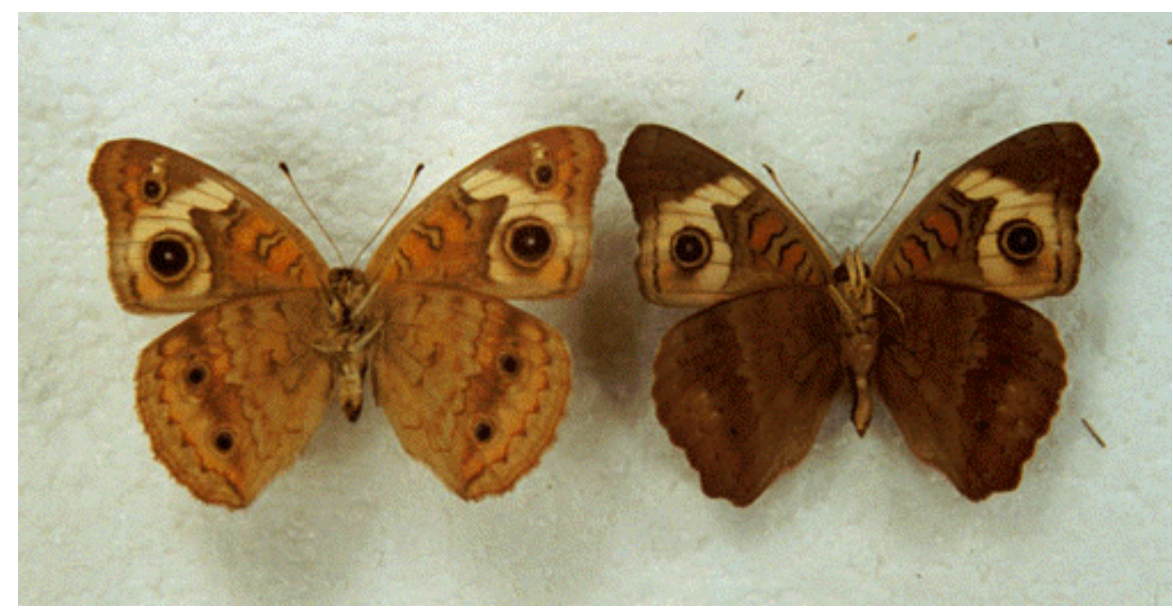

Fig. 2. Polyphenisms in insects are usually contrasting and dramatic

a) Seasonal morphs of the buckeye butterfly, Precis coenia: The ventral surfaces exemplify seasonal polyphenism in Lepidoptera. The summer morph ("linea") is on the left; the Fall morph ("rosa") is on the right. Image courtesy of Scott F. Gilbert.

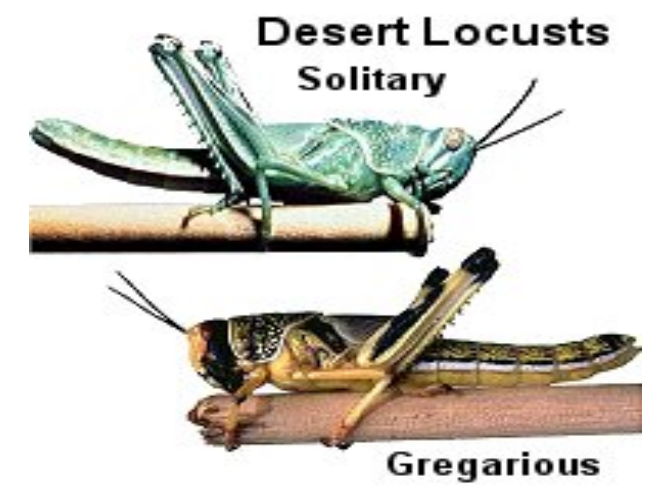

b) The desert locust (Schistocerca gregaria) nymph can transform into the solitary adult morph or the gregarious adult morph under the appropriate environmental conditions, i.e., crowding. Photograph courtesy Compton Tucker, NASA GSFC. 


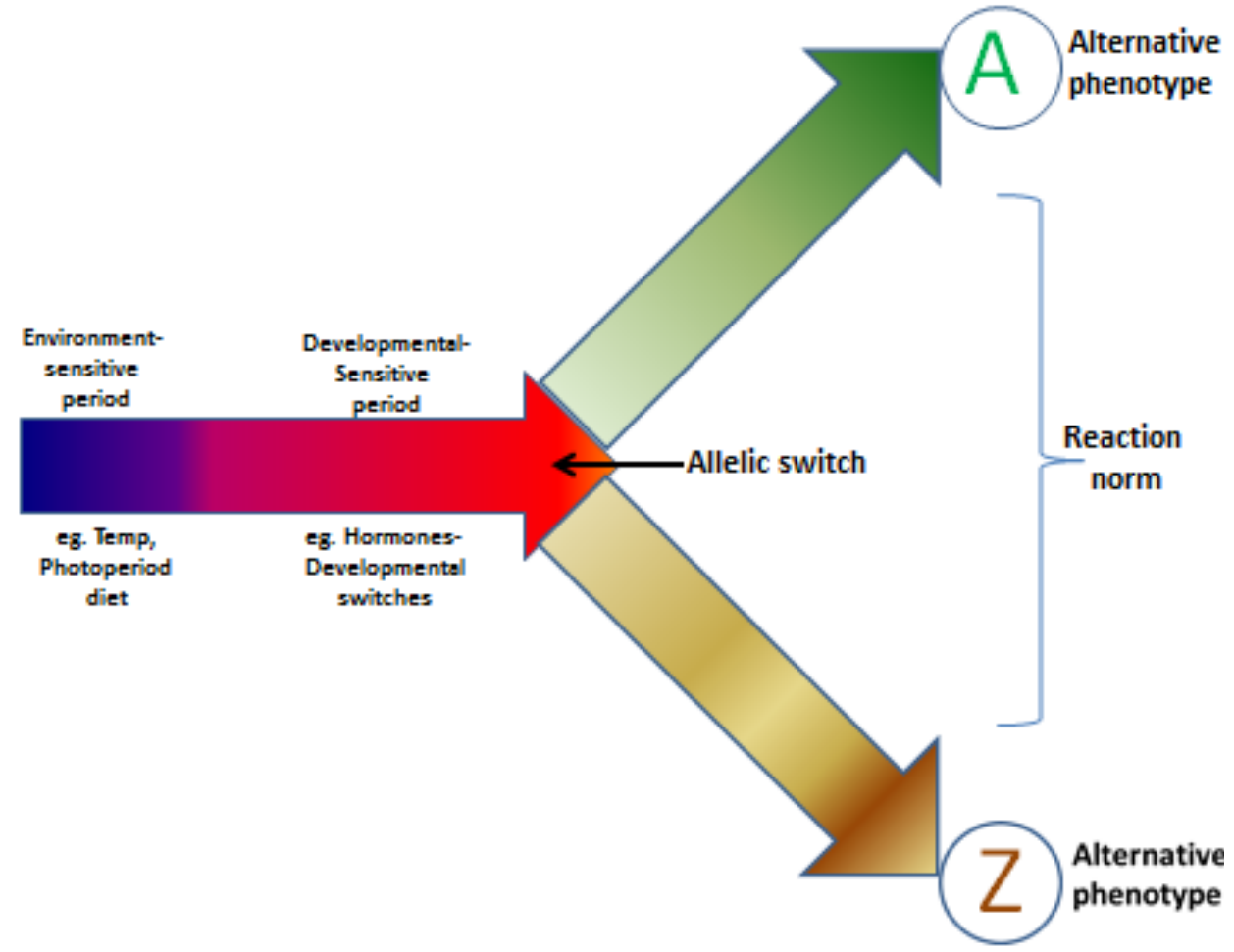

Fig. 3. A generalized schematic representation of the control mechanisms of phenotypic plasticity: Environment, development and genetics can all contribute to either, a) distinct alternative phenotypes ( $\mathrm{A}$ or $\mathrm{Z}$ ) or $\mathrm{b}$ ) a continuous range of phenotypes in the form of a reaction norm. Some polyphenisms arise when reaction norms become discontinuous so that the phenotype no longer changes gradually in correlation with graded changes in the environment. A polyphenism can be thought of as a reaction norm with a threshold, such that a discrete phenotypic switch occurs at small segment of the entire range of environmental variation. 


\subsection{References}

Alberts, B., J. A., et al. (2002). Molecular Biology of the Cell in Cell 4th, Garland Science.

Alto, B. W. and L. P. Lounibos (2013). Vector competence for arboviruses in relation to the larval environment of mosquitoes. W. T. a. C. J. M. Koenraadt, Wageningen Academic Pub: 81 .

Alto, B. W., L. P. Lounibos, et al. (2005). "Larval Competition Differentially Affects Arbovirus Infection in Aedes Mosquitoes." Ecology 86(12): 3279-3288.

Alto, B. W., L. P. Lounibos, et al. (2008). "Larval competition alters susceptibility of adult Aedes mosquitoes to dengue infection." Proceedings of the Royal Society B: Biological Sciences 275(1633): 463-471.

Alto, B. W., E. J. Muturi, et al. (2012). "Effects of nutrition and density in Culex pipiens." Medical and Veterinary Entomology: no-no.

Alto, B. W., M. H. Reiskind, et al. (2008). Size Alters Susceptibility of Vectors to Dengue Virus Infection and Dissemination. 79: 688-695.

Amiard, J. C., C. Amiard-Triquet, et al. (2006). "Metallothioneins in aquatic invertebrates: Their role in metal detoxification and their use as biomarkers." Aquatic Toxicology 76(2): 160-202.

Andrews, G. K. (2000). "Regulation of metallothionein gene expression by oxidative stress and metal ions." Biochem Pharmacol 59: 95-104.

Barrera, R., M. Amador, et al. (2006). "Ecological Factors Influencing Aedes aegypti (Diptera: Culicidae) Productivity in Artificial Containers in Salinas, Puerto Rico." Population And Community Ecology 43(3): 484-92.

Barrera, R., M. Amador, et al. (2008). Unusual productivity of Aedes aegypti in septic tanks and its implications for dengue control. Medical and Veterinary Entomology. 22: 62-69.

Brakefield, P. M., W. A. Frankino, et al. (2009). Polyphenisms in Lepidoptera: multidisciplinary approaches to studies of evolution and development. Phenotypic plasticity of insects: mechanisms and consequences D. W. Whitman and T. N. Ananthakrishnan, Science Publishers, Inc.: 337-368.

Brakefield, P. M., J. Gates, et al. (1996). Development, plasticity and evolution of butterfly eyespot patterns. Nature. 384: 236-242. 
Briegel, H. (1990). "Metabolic relationship between female body size, reserves, and fecundity of Aedes aegypti." Journal of Insect Physiology 36(3): 165-172.

Briegel, H., M. Hefti, et al. (2002). "Lipid metabolism during sequential gonotrophic cycles in large and small female Aedes aegypti." Journal of Insect Physiology 48(5): 547-554.

Caroci, A. S., Y. Li, et al. (2004). "Reduced juvenile hormone synthesis in mosquitoes with low teneral reserves reduces ovarian previtellogenic development in Aedes aegypti." Journal of Experimental Biology. 207(15): 2685-2690.

Chinery, W. A. (1995). "Impact of rapid urbanization on mosquitoes and their disease transmission potential in Accra and Tema, Ghana." Afr. J. Med. med. Sci 24: 179188.

Christophers, R., Ed. (1960). Aëdes aegypti (L.) the Yellow Fever Mosquito: its Life History, Bionomics and Structure, Cambridge University Press.

Clements, A. N., Ed. (1992). The Biology of Mosquitoes. London, Chapman and Hall.

Clifton, M. E. and F. G. Noriega (2011). "Nutrient limitation results in juvenile hormonemediated resorption of previtellogenic ovarian follicles in mosquitoes." Journal of Insect Physiology 57(9): 1274-1281.

Clifton, M. E. and F. G. Noriega (2012). "The fate of follicles after a blood meal is dependent on previtellogenic nutrition and juvenile hormone in Aedes aegypti." Journal of Insect Physiology 58(7): 1007-1019.

Crovello, T. J. and C. S. Hacker (1972). "Evolutionary Strategies in Life Table Characteristics Among Feral and Urban Strains of Aedes aegypti (L.)." Evolution 26(2): 185-196.

Denlinger, D. L. (1986). "Dormancy in tropical insects." Annual Review of Entomology 31(1): 239-264.

Dufty, A. M., J. Clobert, et al. (2002). "Hormones, developmental plasticity and adaptation." Trends in Ecology \& Evolution 17(4): 190-196.

Egli, D., J. Domenech, et al. (2006). "The four members of the Drosophila metallothionein family exhibit distinct yet overlapping roles in heavy metal homeostasis and detoxification." Genes to Cells 11(6): 647-658. 
Egli, D., H. Yepiskoposyan, et al. (2006). "A Family Knockout of All Four Drosophila Metallothioneins Reveals a Central Role in Copper Homeostasis and Detoxification." Molcular and Cellular Biology 26(6): 2286-2296.

Endo, K., I. Yamashita, et al. (1985). Effect of photoperiodic transfer and brain surgery on the photoperiodic control of pupal diapause and seasonal morphs in the swallowtail, Papilio xuthus L.(Lepidoptera: Papilionidae). Applied Entomology and Zoology. 20: 470-478.

Esperk, T., C. Stefanescu, et al. (2012). "Distinguishing between anticipatory and responsive plasticity in a seasonally polyphenic butterfly." Evolutionary Ecology: $1-18$.

Fairbairn, D. J. and D. E. Yadlowski (1997). "Coevolution of traits determining migratory tendency: correlated response of a critical enzyme, juvenile hormone esterase, to selection on wing morphology." Journal of Evolutionary Biology 10(4): 495-513.

Foster, W. A. (1995). "Mosquito sugar feeding and reproductive energetics " Annual Review of Entomology 40: 443-474.

Frankie, G. W. and L. E. Ehler (1978). Ecology of Insects in Urban Environments. 23: 367-387.

Gaetke, L. M. and C. K. Chow (2003). "Copper toxicity, oxidative stress, and antioxidant nutrients. ." Toxicology (189): 147-163.

Garland, T. and S. A. Kelly (2006). "Phenotypic plasticity and experimental evolution." Journal of Experimental Biology 209(12): 2344-2361.

Goodman, W. G. and M. Cusson (2012). The Juvenile Hormones. Insect Endocrinology L. Gilbert, Academic Press.

Hahn, D. A. and D. L. Denlinger (2007). "Meeting the energetic demands of insect diapause: Nutrient storage and utilization." Journal of Insect Physiology 53(8): 760-773.

Hare, L. (1992). "Aquatic insects and trace metals: Bioavailabilty, Bioaccumulation and Toxicity." Critical Reviews in Toxicology 22(5/6): 327-369.

Ishikawa, A., H. Gotoh, et al. (2013). "Juvenile hormone titer and wing-morph differentiation in the vetch aphid Megoura crassicauda." Journal of Insect Physiology 59(4): 444-449.

Kagi, J. H. R. (1991). "Overview of metallothionein." Methods Enzymol 205: 613-626. 
Koch, P. B. and D. Buckmann (1987). Hormonal control of seasonal morphs by the timing of ecdysteroid release in Araschnia levana, (Nymphalidae: Lepidoptera). Journal of Insect Physiology, Elsevier. 33: 823-829.

Maroni, G. W., J. Young, J. E. Otto, E. (1987). "Metallothionein Gene Duplications and Metal Tolerance in Natural Populations of Drosophila melanogaster." Genetics 117. (4): 739-744.

Mayr, E., Ed. (1963). Animal Species and Evolution, Cambridge: Belknap Press of Harvard University Press.

Mireji, P. O., J. Keating, et al. (2008). "Heavy metals in mosquito larval habitats in urban Kisumu and Malindi, Kenya, and their impact." Ecotoxicology and Environmental Safety 70(1): 147-153.

Moran, N. A. (1992). "The Evolutionary Maintenance of Alternative Phenotypes." The American Naturalist 139(5): 971-989.

Nene, V., J. R. Wortman, et al. (2007). "Genome sequence of Aedes aegypti, a major arbovirus vector." Science 316(5832): 1718.

Nijhout, H. F. (1994). Insect Hormones, Princeton University Press.

Nijhout, H. F. (1999). "Control Mechanisms of Polyphenic Development in Insects." BioScience 49(3): 181-192.

Ocampo, C. B. and D. M. Wesson (2004). "Population Dynamics of Aedes aegypti from a Dengue Hyperdermic Urban Setting in Columbia." Am. J. Trop. Med. Hyg 71(4): 506-513.

O'Meara, G. E., Leonard F. Gettman, Alan D. (1992). "Reduced Mosquito Production in Cemetary Vases with Copper Liners." Operational and Scientific Notes 8(4): 419420.

O'Meara, G. F. and L. P. Lounibos (1981). "Reproductive maturation in the pitcher-plant mosquito, Wyeomyia smithii." Physiological Entomology 6(4): 437-443.

Posthuma, L. and N. M. Van Straalen (1993). "Heavy-metal adaptation in terrestrial invertebrates : a review of occurrence, genetics, physiology and ecological consequences." Comparative biochemistry and physiology. C. Comparative pharmacology and toxicology 106(1): 11-38

Poupardin, R., S. Reynaud, et al. (2008). "Cross-induction of detoxification genes by environmental xenobiotics and insecticides in the mosquito Aedes aegypti: Impact 
on larval tolerance to chemical insecticides." Insect Biochemistry and Molecular Biology 38(5): 540-551.

Puig, S. and D. J. Thiele (2002). "Molecular mechanisms of copper uptake and distribution." Current Opinion in Chemical Biology 6(2): 171-180.

Riddiford, L. M. (1996). Molecular aspects of juvenile hormone action in insect metamorphosis. Metamorphosis: Postembryonic Reprogramming of Gene Expression in Amphibian and Insect Cells, Acdemic Press Inc. : 223-251.

Roskam, J. C. and P. M. Brakefield (1996). "A Comparison of Temperature-Induced Polyphenism in African Bicylus Butterflies from a Seasonal Savannah-Rainforest Ecotone." Evolution 50(6): 2360-2372.

Rountree, D. B. and H. F. Nijhout (1995). "Hormonal control of a seasonal polyphenism in Precis coenia (Lepidoptera: Nymphalidae)." Journal of Insect Physiology 41(11): 987-992.

Sarkar, S., A. K. Duttagupta, et al. (2004). "Effects of heavy metals on population growth and metallothionein gene expression in the mosquito Culex quinquefasciatus, from Calcutta, India." Environmental Pollution 127(2): 183-193.

Scheiner, S. M. and R. F. Lyman (1991). "The genetics of phenotypic plasticity. II. Response to selection." Journal of Evolutionary Biology 4(1): 23-50.

Schlichting, C. D. and M. Pigliucci (1995). Gene regulation, quantitative genetics and the evolution of reaction norms. Evolution Springer. 9: 154-168.

Shapiro, A. M. (1976). Seasonal Polyphenism. Evolutionary Biology. M. K. Hecht, W. C. Steere and B. Wallace, Springer US: 259-333.

Shirley, M. D. F. and R. M. Sibly (1999). "Genetic Basis of a between-Environment Trade-off Involving Resistance to Cadmium in Drosophila melanogaster." Evolution 53(3): 826-836.

Simpson, S., G. Sword, et al. (2011). "Polyphenism in Insects." Current Biology 21(18): R738-R749.

Smith, D. L., K. E. Battle, et al. (2009). "Ross, Macdonald, and a Theory for the Dynamics and Control of Mosquito-Transmitted Pathogens." PLoS Pathog 8(4): e1002588.

Smith, K. C. (1991). The effects of temperature and daylength on the rosa polyphenism in the buckeye butterfly, Precis coenia (Lepidoptera: Nymphalidae). Journal of Research on Lepidoptera 30: 225-236. 
Staal, G. B. (1961). Studies on the Physiology of Phase Induction in Locusta Migratoria Migratorioides R. and F, H. Veenman and Zonen.

Stearns, S. C. (1989). "The evolutionary significance of phenotypic plasticity." BioScience 39(7): 436-445.

Tauber, M., J., C. A. Taube, et al. (1986). Seasonal adaptations of insects, Oxford University Press, USA.

Vinogradova, E., V. Alekseev, et al. (2007). Diapause in Aquatic Insects, with Emphasis on Mosquitoes

Diapause in Aquatic Invertebrates Theory and Human Use. H. J. Dumont and M. J. A. Werger, Springer Netherlands. 84: 83-113.

West-Eberhard, M. (2003). Developmental Plasticity and Evolution, Oxford University Press, USA.

West-Eberhard, M. J. (1986). "Alternative adaptations, speciation, and phylogeny (A Review)." Proceeds of the National Acedemy of Science 83(5): 1388-1392.

West-Eberhard, M. J. (1989). "Phenotypic Plasticity and the Origins of Diversity." Annual Review of Ecology and Systematics 20: 249-278.

Wheeler, D. E. and H. Frederik Nijhout (1984). "Soldier determination in Pheidole bicarinata Inhibition by adult soldiers." Journal of Insect Physiology 30(2): 127135.

Wu, R., C.-X. Ma, et al. (2003). "Molecular Dissection of Allometry, Ontogeny, and Plasticity: A Genomic View of Developmental Biology." BioScience 53(11): 1041-1047.

Zera, A. J. (2006). "Evolutionary genetics of juvenile hormone and ecdysteroid regulation in Gryllus : A case study in the microevolution of endocrine regulation." Comparative Biochemistry and Physiology-Part A 144(3): 365-379.

Zera, A. J. and R. F. Denno (1997). "Physiology and ecology of dispersal polymorphism in insects." Annual Review of Entomology 42(1): 207-230.

Zera, A. J., C. Strambi, et al. (1989). "Juvenile hormone and ecdysteroid titres during critical periods of wing morph determination in Gryllus rubens." Journal of Insect Physiology 35(6): 501-511.

Zera, A. J. and K. C. Tiebel (1989). "Differences in juvenile hormone esterase activity between presumptive macropterous and brachypterous Gryllus rubens: 
Implications for the hormonal control of wing polymorphism." Journal of Insect Physiology 35(1): 7-17.

Zera, A. J. and C. Zhang (1995). "Evolutionary endocrinology of juvenile hormone esterase in Gryllus assimilis: direct and correlated responses to selection." Genetics 141(3): 1125. 


\section{Chapter 3: The sub-lethal larval metal stress response of the Dengue Fever Mosquito}

\subsection{Abstract}

Aedes aegypti (Diptera: Culicidae, Linnaeus) has adapted to urban environments; the urbanization process provides suitable habitats for this disease vector subsequently increasing the probability of the transmission of pathogens in high-density environments. Urban environments provide metal stressed larval habitats; therefore, metal tolerance physiology needs to be considered with respect to the adaptations this disease vector is undergoing. Aedes aegypti, being a container breeder, is subject to greater physiological stress of metals in the environment than other species of mosquitoes. Little is known about the physiological cost of metal stress or how this might affect the fitness of the mosquito population and their vector capacity. This study sought to characterize some of the sub-lethal physiological consequences of metal stress in A. aegypti. To carry this out various parameters of mosquito physiology under larval metal stress were assessed, including larval metallothionein expression and the effects of larval metal stress on adults and their progeny. These findings indicate that even low levels of environmentally relevant larval metal stress have wide and far reaching physiological consequences for this important disease vector, suggesting that $A$. aegypti exposed to urban metal pollution is likely at higher risk of increased vector capacity than their sylvatic counterparts.

\subsection{Introduction}

Increasing urban populations throughout developing countries have been accompanied by increasing industrial activities, indiscriminant use of pesticides, a lack of environmental regulation and an increase in poorly planned cities, all resulting in 
environmental pollution (Montgomery, 2008). The presence of Aedes aegypti larvae have been confirmed in polluted habitats such as drains containing domestic wastewater and man-made aquatic habitats (Barrera et al., 2008), habitats previously not associated with this species of mosquito. Aedes aegypti is the primary vector of dengue and yellow fevers and these observations suggest that pollutant tolerant strains of Aedes species of mosquitoes are developing. Aedes aegypti has, in fact, adapted to urban environments; domestic and feral forms have been recognized in Africa (Crovello and Hacker, 1972). The urbanization process provides suitable habitats for this mosquito subsequently increasing the probability of the transmission of diseases in high-density urban environments (Frankie and Ehler, 1978). Larval breeding sites are of particular interest i.e., septic tanks, discarded metal cans, cisterns, drains, sewers, tires and dumpsters. In urban environments there are many sources of metal pollution (run off, fall out, indiscriminant use of metal containing herbicides, etc.) Therefore, metal tolerance needs to be considered with respect to the adaptations this disease vector is undergoing (Posthuma and Van Straalen, 1993; Poupardin, et al., 2008). Adaptations to novel environments provide distinct advantages; however, performance costs are associated with them. These costs, in turn, will affect the disease transmission dynamics of a vector i.e., vector capacity (Alto and Lounibus, 2012). Anthropogenic changes to the environment such as urbanization and pollution can result in physiological changes in disease vectors with potential epidemiological consequences.

The Yellow Fever Mosquito, being a container breeder, is subject to greater physiological stress of metals in the environment than other species of mosquitoes that transmit disease. Little is known about the physiological cost of metal pollution or how 
this might affect the fitness of the mosquito population and their ability to transmit diseases (Mireji et al., 2010; Rayms-Keller et al., 1998; Sarkar et al., 2004). Of the many metals that $A$. aegypti mosquitoes are exposed to in an urban environment, copper $\left(\mathrm{Cu}^{2+}\right)$ is of primary interest. It used indiscriminately as an algaecide, fungicide, root treatment agent and in the manufacture of artificial containers (Barrera et al., 2008; Mireji et al., 2008). It is an essential trace element with an important role in several cellular processes such as respiration, oxidative stress defense and immune function and is found in a number of important metabolic enzymes such as cytochrome $c$ oxidase, $\mathrm{Cu}-\mathrm{Zn}$ superoxide dismutase 1 (SOD1), tyrosinase, lysyl oxidase and multicopper oxidase (Puig and Theile, 2002). Aedes aegypti mosquitoes need to regulate this toxic agent that at the same time is essential to basal metabolism. An investigation of $\mathrm{Cu}^{2+}$ tolerance physiology can also serve as a model for mercury, silver, zinc and cadmium as each of these share similar detoxification pathways via metallothionein binding. Metallothioneins are small, ubiquitous, cysteine rich proteins with a high affinity for transition metals and have an important role in the homeostasis of essential metals, the detoxification of nonessential metals and the oxidative stress response (Amiard, et al., 2006). It would be, therefore, useful to have an understanding of $A$. aegypti metal toxicity and tolerance.

This study sought to determine how sensitive $A$. aegypti is to larval metal stress and to characterize some of its sub-lethal physiological consequences. To carry this out various parameters of mosquito physiology under larval metal stress were assessed, including larval metallothionein expression and the effects of larval metal stress on adults and their progeny. These findings indicate that even low levels of environmentally relevant larval metal stress have wide and far reaching physiological consequences for 
this important disease vector, suggesting that $A$. aegypti exposed to urban metal pollution are likely at higher risk of increased vector capacity than their sylvatic counterparts.

\subsection{Materials and Methods}

\subsubsection{Mosquito rearing and maintenance}

Aedes aegypti (Rockefeller strain) pharate first instars (eggs less than a week old) were hatched in deoxygenated water. Upon emergence, three replicate groups of 200 larvae were reared to adulthood in $23 \times 40 \times 15 \mathrm{~cm}$ polypropylene plastic pans containing $1 \mathrm{~L}$ of reconstituted soft water (RSW). For the metal stressed treatment, three replicate groups of 200 first instar larvae were reared to adulthood in one ppm copper $\left(\mathrm{Cu}^{2+}\right)$ in RSW. One ppm $\mathrm{Cu}^{2+}$ has been shown to be an ecologically relevant level of metal stress (Sarkar, et al., 2004, Mireji, et al., 2008). Larval diet consisted of $750 \mu 1$ of $10 \%$ liver powder suspension (MP Biomedicals Inc., Aurora, OH.) added to the pan every day except for the second day. Pupae were collected every day throughout the pupation period and transferred to an appropriate container for adult emergence. Adult mosquitoes were offered a cotton pad soaked in 3\% sucrose solution ad libitum. Four-day-old female mosquitoes were fed porcine blood equilibrated to $37^{\circ} \mathrm{C}$ and ATP added to the blood meal to a final concentration of $1 \mathrm{mM}$ immediately before use. Eggs were oviposited on papers and stored under insectary conditions in Nasco Whirl-Pak bags inside a re-sealable polypropylene container. Insectary conditions were $28{ }^{\circ} \mathrm{C}$ and $80 \%$ relative humidity under a photoperiod of 16 hour light and 8 hour dark. All assays were performed under insectary conditions $\sim 12: 00 \mathrm{pm}$. 


\subsubsection{Reagents}

Reconstituted soft water (RSW) was used in all bioassays. The RSW was prepared by adding reagent-grade $\mathrm{NaHCO}_{3}(48 \mathrm{mg}), \mathrm{CaSO}_{4} 2 \mathrm{H}_{2} \mathrm{O}(30 \mathrm{mg}), \mathrm{MgSO}_{4}(30$ $\mathrm{mg})$ and $\mathrm{KCl}(2 \mathrm{mg})$ to $1 \mathrm{~L}$ of deionized water $(\mathrm{pH} \sim 7.4)$. All reagents were purchased from Fisher Scientific, Pittsburgh, PA.

\subsubsection{Copper measurement}

Reagent grade copper sulfate pentahydrate was dissolved in RSW. Nominal copper $\left(\mathrm{Cu}^{2+}\right)$ concentrations were confirmed by inductively coupled plasma mass spectrometric analysis (ICP-MS) using an HP 4500 plus IPC-MS instrument (HewlettPackard Co., Wilmington, DE), equipped with a Babington-type nebulizer and an ASX500 autosampler (Cetac Technologies Inc., Omaha, NE).

\begin{tabular}{|l|l|l|l|l|}
\hline & 1ppm & $\mathbf{2 0 0 0} \mathbf{p p b}$ & $\mathbf{3 0 0 0} \mathbf{p p b}$ & $\mathbf{4 0 0 0} \mathbf{p p b}$ \\
\hline & AA Cu std & test soln & test soln & test soln \\
\hline & dil to 50ppb & df $=40$ & df $=60$ & df $=53.33$ \\
\hline & df = 20000 & & & \\
\hline mean & $\mathbf{5 3 . 0 5}$ & $\mathbf{5 2 . 9 7}$ & $\mathbf{5 4 . 3 3}$ & $\mathbf{6 3 . 6 2}$ \\
\hline std dev & 0.36 & 0.32 & 0.35 & 0.31 \\
\hline & $6 \%$ error & $6 \%$ error & $9 \%$ error & $15 \%$ error \\
\hline
\end{tabular}

\subsubsection{Images of first instars and adult tissues}

Representative individuals of 24 hour (h) old first instars and three day old adult females for each treatment were imaged with a Leica MZ6 stereo dissecting microscope equipped with a Leica DC500 digital camera and LAS 2.8.1 software at 40x magnification. All images were processed with Photoshop C2 software from Adobe to 
remove background glare and reflection, the actual larval and tissue component of the images were never altered.

\subsubsection{Dose response curve}

Early $3^{\text {rd }}$ instar larvae reared under standard conditions in clean RSW were exposed to increasing concentrations of $\mathrm{Cu}^{2+}$ (from $\left.\mathrm{CuSO}_{4}\right)(\mathrm{n}=25$, in triplicate) for $24 \mathrm{~h}$. Lowest observed effect concentration (LOEC) and $\mathrm{LC}_{50}$ values were determined by $\log$ transforming the $\mathrm{Cu}^{2+}$ concentration and fitting a sigmoidal nonlinear curve.

\subsubsection{Metal tolerance}

Increased tolerance to metal stress was demonstrated by rearing larvae in 0.75 parts per million (ppm) $\mathrm{Cu}^{2+}$ or in clean $\mathrm{RSW}$. When larvae from each treatment reached early $3^{\text {rd }}$ instar stage they were subjected to an acute toxicity assay in a time response manner by exposing them to the $\mathrm{LC}_{50}$ concentration determined by the dose response curve from the previous section until $100 \%$ mortality had occurred ( $n=20$, in duplicate). Median $\mathrm{LT}_{50}$ values were determined by generating a survivorship curve and performing a log rank test (Mantel-Cox).

\subsubsection{Quantification of total neutral lipids}

Newly emerged 3rd instar larvae were exposed to $1.25 \mathrm{ppm}^{2+} \mathrm{uu}^{2+} \mathrm{RSW}$ under insectary conditions in the absence of food for $5 \mathrm{~d}$. During that time the larvae molted to the $4^{\text {th }}$ instar stage. Negative control larvae were held in clean RSW. Neutral lipids were quantified using a triglyceride quantification kit (Biovision, Mountain View, CA; cat\#

K622-100). Three $4^{\text {th }}$ instars were assayed per biological replicate in triplicate $(N=9)$. To 
stain neutral lipids larvae were fixed in $4 \%$ formaldehyde overnight, washed with cold PBS, stained with Oil Red-O for 30 min., washed with cold PBS and imaged with a Leica Model MZ6 stereomicroscope at 40x magnification (adapted from Nishiura et al., 2007).

\subsubsection{Fitness assays}

To determine adult body mass newly emerged adult females from each treatment were collected, dried at $95^{\circ} \mathrm{C}$ for $24 \mathrm{~h}$ and analyzed gravimetrically with a Cahn 29 electrobalance to within one $1 / 100$ of a milligram. For the starvation tolerance assay three replicate groups of 15 newly emerged adult females from each treatment were assayed in one pint mosquito cages and provided deionized water ad libitum. The longevity assay was performed in a similar manner except $3 \%$ sucrose was provided. For the filial larvae starvation tolerance assay (maternal effects) three replicate groups of 25 newly emerged first instars generated from mosquitoes reared under the above mentioned "Mosquito rearing and maintenance" protocol were assayed in $15 \mathrm{~cm}$ diameter glass petri dishes containing $100 \mathrm{ml}$ of RSW in the absence of food. The number of larvae found not responsive to mechanical stimulation every $24 \mathrm{~h}$ in each assay were counted as "dead" and removed.

\subsubsection{Quantification of follicles per ovary}

A sub-sample of adults was analyzed for follicle quantification. $60-72 \mathrm{~h}$ old adult females were anesthetized by chilling for $10 \mathrm{~min}$ at $4^{\circ} \mathrm{C}$ prior to dissection. Ovaries from these mosquitoes were removed, rinsed in Aedes physiological saline (APS) $\left(\mathrm{MgCl}_{2} 0.6\right.$ mM; KCl 4.0 mM NaHCO 31.8 mM; NaCl 150.0 mM; HEPES 25.0 mM; $\mathrm{CaCl}_{2} 1.7 \mathrm{mM}$ ) 
and stained with $0.5 \%$ neutral red solution in acetate buffer at pH 5.2 (Sigma-Aldrich, St. Louis, MO) for $5 \mathrm{~s}$ to more easily visualize follicles (Clifton and Noriega, 2011). The ovaries were rinsed a second time in APS and placed under a coverslip. Photographs were taken of the previtellogenic ovaries using a DM 5500 B Leica fluorescence microscope, a Leica DFC 310 FX mounted camera and Leica LAS imaging software. Ovaries were later scored using Leica LAS imaging software for total follicle count. A mean follicle value was then calculated to represent previtellogenic follicle output for the cohort.

\subsubsection{A. aegypti metallothionein (AaMtn) induction}

To induce $A a \mathrm{Mtn}$ transcription newly emerged first instar larvae hatched from

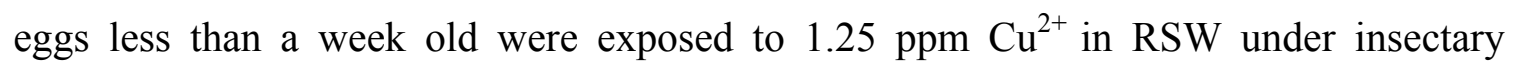
conditions in the absence of food for $12 \mathrm{~h}$. To demonstrate the reversible nature of $A a \mathrm{Mtn}$ transcription metal exposed larvae were transferred back to clean water after $6 \mathrm{~h}$ of metal exposure. Three independent biological replicates for each time point consisting of $~ 50$ larvae derived from three different cohorts of eggs were collected and stored at minus $80^{\circ} \mathrm{C}$.

\subsubsection{Midgut as a barrier to metal toxicity}

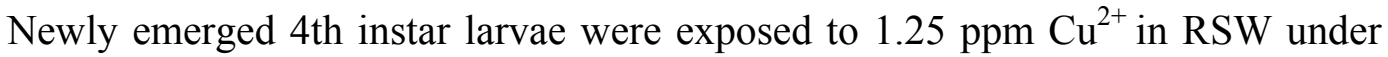
insectary conditions in the absence of food for $6 \mathrm{~h}$. Negative control larvae were held in clean RSW. Fourth instar midgut tissues were separated from the rest of the body $(n=5)$ (triplicate) and both midguts and 'rest of body' tissues were stored in RNAlater 
(Ambion) at minus $80^{\circ} \mathrm{C}$. Midgut $\mathrm{AaMtn}$ transcription level was analyzed and compared to the rest of the body tissue.

\subsubsection{Quantitative real-time PCR ( $q P C R)$}

Total RNA was isolated from samples using RNA-binding glass powder as previously described (Noriega and Wells, 1993). Contaminating genomic DNA was removed using the DNA-free ${ }^{\mathrm{TM}}$ kit (Ambion, Austin, TX). Reverse transcription was carried out using the Verso cDNA Kit (Fisher Scientific). Real time qPCR was performed with the 7300 Real Time PCR System using TaqMan ${ }^{\circledR}$ Gene Expression Assays together with TaqMan ${ }^{\circledR}$ Universal PCR Master Mix (Applied Biosystems, Foster City, CA). The primer probes for housekeeping gene 60S ribosomal protein L32 (rpL32) (Accession number: AAEL003396) and for metallothionein (AaMtn) (Accession number: AAEL008176) were as follows (exon borders in bold):

rpL32 Forward: 5' CCAAGCCAGATTCCTAACATTCAAA 3'

rpL32 Reverse: 5' TGCCCTGTCCGCAACC 3'

rpL32 Probe: 5' CTTGCAATCATTACCACAGCAC 3'

Mtn Forward: 5' GCCAAGCCAGATTCCTAACATTCAA 3'

Mtn Reverse: 5' GCAACCGGAGGTGCACTT 3'

Mtn Probe: 5' AagtgCtgtggtaAtgattgC 3'

qPCR reactions were carried in a $20 \mu \mathrm{l}$ volume according to manufacturer's recommendations for Custom TaqMan ${ }^{\circledR}$ Gene Expression Assays. Reactions were run in triplicate using $1 \mu \mathrm{l}$ of cDNA per reaction. Standard curves to quantify relative gene copy number were made from serial dilutions of plasmids containing rpL32 or $A a \mathrm{Mtn}$ gene 
$(300,000,30,000,3000,300$ and 30 copies of a plasmid per reaction). Real time data were collected by 7300 System SDS Software and analyzed in Microsoft Excel. AaMtn transcript levels were normalized with rpL32 transcript levels in the same sample. Relative $A a \mathrm{Mtn}$ transcript levels are expressed as a number of copies of $A a \mathrm{Mtn}$ transcript per 10,000 copies of rpL32 transcript. Each qPCR data point is the average of three independent biological replicates.

\subsubsection{Data analysis}

Graphical representations and statistical analysis were performed with GraphPad Prism Software (San Diego, CA) version 6.0 for Windows. Results were expressed as means and SEM. Survivorship $\left(\mathrm{LT}_{50}\right)$ was determined by non-linear regression curve fit. Significant differences in survivorship $\left(\mathrm{LT}_{50}\right)$ were determined with a log rank method. Significant differences for all other results were determined with either a one tailed student's $\mathrm{t}$ test or a one way ANOVA followed by a Tukey's test for multiple comparisons.

\subsection{Results}

\subsubsection{Larval metal stress altered larval and adult phenotypes}

Aedes aegypti larvae reared under the metal stressed treatment exhibited a visibly altered phenotype than those that had been reared in a clean environment with no metal stress. At $24 \mathrm{~h}$ of development first instar larvae were distinctly different in not only size but general overall appearance (Fig. 4A and B). The difference in size is likely the effect

of clean reared larvae having molted to the $2^{\text {nd }}$ instar while metal stressed larvae remain 
in first instar stage longer. Previous work has demonstrated that metal stressed larvae exhibit longer developmental time. In the adult stage, there were also dramatic visible differences between treatments (Fig. 1C). Most notably, ovaries and malpighian tubules of three day old females were affected. Overall, metal stressed larvae and their corresponding adult tissues were less robust, pale and translucent.

\subsubsection{A. aegypti larvae were sensitive to metal stress}

Aedes aegypti $3^{\text {rd }}$ instars responded to $\mathrm{Cu}^{2+}$ in a dose dependent manner in a $24 \mathrm{~h}$ acute toxicity assay (Fig. 5). The lowest observed effect concentration (LOEC) was 1.6 $\mathrm{ppm}$ and the lethal concentration of $50 \%$ of the sample population $\left(\mathrm{LC}_{50}\right)$, was $2.06 \mathrm{ppm}$. Aedes aegypti was found to be sensitive to $\mathrm{Cu}^{2+}$ and exhibited a narrow response range $(\sim 3 \mathrm{ppm})$, i.e., the difference between the LOEC and the concentration that resulted in $100 \%$ mortality.

\subsubsection{A. aegypti larvae exhibited tolerance}

Larvae reared under the metal stress treatment to the $3^{\text {rd }}$ instar stage exhibited significantly longer survivorship (median $\mathrm{LT}_{50}, 44 \mathrm{vs} .14 \mathrm{~h}$ ) in an acute toxicity assay than larvae reared in clean water. Previous metal exposure also resulted in a shift from a Type III survivorship curve (clean reared) to a Type II survivorship curve (metal stressed) suggesting altered detoxification dynamics (Fig. 6). In the Type III curve the greatest mortality is experienced early in the assay with a relatively low rate of mortality late in the assay suggesting that survivorship is dependent on two modes of actions. A 
type II curve assumes an approximately constant mortality rate indicative of one mode of action.

\subsubsection{Metal stress and neutral storage lipids}

There was a nutritional cost associated with metal stress. Larvae held in $1.25 \mathrm{ppm}$ $\mathrm{Cu}^{2+}$ for 5 days (d) resulted in a 58\% greater reduction in neutral lipid reserves compared to those held in clean water (Fig. 7). Fourth instar larvae stained with Oil Red O visually confirmed this reduction in neutral lipid reserves (Fig. 4 inset).

\subsubsection{AaMtn was expressed predominately in the midgut of larvae}

AaMtn expression in midguts of larvae held in clean water was $\sim 20$ fold higher than AaMtn expression in the rest of the body representing the basal metabolic state of AaMtn without any metal stress; however, under metal stress (4th instars exposed to 1.25

ppm $\mathrm{Cu}^{2+}$ for $6 \mathrm{~h}$ ) midgut $A a \mathrm{Mtn}$ expression was almost $\sim 40$ fold greater than the rest of the body indicating that $A a \mathrm{Mtn}$ is up regulated in the midgut (Fig. 8A). Midguts of larvae exposed to metal stress expressed approximately twice the AaMtn than midguts of larvae held in clean water. However, AaMtn expression in metal stressed body tissues and body tissues exposed to clean water were virtually the same (Fig. 8B).

\subsubsection{AaMtn transcription of first instars was highly dynamic}

Newly emerged first instars exposed to $1.25 \mathrm{ppm}^{\mathrm{Cu}^{2+}}$ for $12 \mathrm{~h}$ expressed $\mathrm{AaMtn}$ at extremely high relative levels compared to larvae held in clean water (121 fold increase at $6 \mathrm{~h}$ and 12 fold increase at $12 \mathrm{~h}$ ) (Fig. 9). The reduction in relative increase 
was mostly a result of the increase in basal AaMtn expression of larvae held in clean water. Basal AaMtn expression increased tenfold from time points 6 to $12 \mathrm{~h}$. Measurable differences in $A a \mathrm{Mtn}$ expression were observed within 30 min. (not shown). AaMtn transcription was also reversible, AaMtn expression dramatically decreased in metal exposed larvae that were transferred back to clean water to levels not significantly different than those held in clean water for the entire $12 \mathrm{~h}$.

\subsubsection{Larval metal stress is reflected in adult phenotype}

Larvae reared under the metal stressed treatment emerged as significantly altered adults. Adult females reared from metal stressed larvae were smaller (17\%) with lowered starvation tolerance (20\%), less neutral lipids (39\%), greater longevity (19\%), fewer follicles $(14 \%)$ and produced filial first instar larvae that also had lowered starvation tolerance (19\%) (Fig.10 A-F).

\subsection{Discussion}

Approximately two-thirds of the world's population lives in areas endemic to dengue vectors, mainly A. aegypti and Aedes albopictus (Janseen and Beebe, 2010); all four dengue virus serotypes are known to affect urban populations and the dengue transmission cycle has been shown to be heavily influenced by environmental conditions (Gubler, 2004). Therefore, a characterization of the metal stress response would be useful in assessing alterations in vector capacity of this important vector as it adapts to urban environments. 


\subsubsection{Metal Tolerance}

Metal tolerance, the physiological adaptation of an individual over the course of its lifetime to metal stress, is the result of the up regulation of detoxification, sequestration and excretion mechanisms and the down regulation of uptake mechanisms. The A. aegypti mosquito is subject to not only the selection pressure of metals in the urban environment, but also suffers the physiological stress associated with it. Aedes aegypti mosquitoes are challenged with the need to protect themselves from potentially toxic metals like $\mathrm{Cu}^{2+}$ that at the same time are essential to basal metabolism (Balamurugan, et al., 2007). The $\mathrm{Cu}^{2+}$ concentrations at which these experiments were conducted ( 1ppm) were ecologically relevant. Various authors have demonstrated environmental metal concentrations in urban habitats to be well above this range (Mireji, et al., 2008; Sarkar, et al., 2004), especially if one were to consider the additive and synergistic effects of multiple metal exposures in natural settings that larvae might encounter. Therefore, the physiological responses observed in this work are not toxic effects from unrealistic laboratory exposures, but rather, they model realistic organismal responses to environmental stress.

The results of this work have demonstrated that the A. aegypti mosquito is sensitive to metal stress and that even a relatively low concentration of metal exposure result in distinctly different larval, adult and $F_{1}$ larval phenotypes with different morphological and physiological features. The observed adult phenotypes resulting from larval metal stress likely results in fitness costs similar to those described by other authors (Kosalwat and Knight, 1987b; Mireji, et al., 2010). The tissues of the adult 
mosquitoes reared under the metal stressed environment were less robust in appearance than their clean reared counterparts and the adults and their progeny displayed overall compromised performance. The pale translucent appearance of the tissues is likely the result of diminished lipids reserves (Perez and Noriega, 2012).

Chronic and sub lethal dose effects have been shown in a variety of other dipteran taxa (Kosalwalt and Knight, 1987a; Mireji, et al., 2010; Marinkovic, et al., 2011). The dose response curve produced under these conditions for $\mathrm{Cu}^{2+}$ exhibited a narrow response range of $\sim 3 \mathrm{ppm}$ that is similar to the work of Kosalwalt and Knight with midges under similar conditions (Kosawalt and Knight 1987b). Rayms-Keller reported a very different $\mathrm{LC}_{50}$ for $\mathrm{Cu}^{2+}$ in $A$. aegypti, but under very different laboratory conditions (Rayms-Keller, et al., 1998). A narrow response range i.e., a steep dose response curve, suggests that there may be only one mechanism of detoxification (Newman and Unger, 2002); once this mechanism is saturated, $\mathrm{Cu}^{2+}$ ions are free to cause oxidative stress and cellular damage resulting in mortality suggesting that there is no redundancy for $\mathrm{Cu}^{2+}$ stress in A. aegytpi.

Chronic sub-lethal exposure to $\mathrm{Cu}^{2+}$ resulted in metal tolerant larvae; this could have occurred by two means, selection or the induction of metal detoxification mechanisms by previous exposure. Previous work has described $\sim 50 \%$ mortality in the metal stress treatment under these very same conditions (Perez and Noriega, 2012) possibly selecting for metal resistance. Censoring the mortality data of the survivorship curve (Fig. 3) of the clean reared treatment to compensate for larvae selected against during rearing results in a shift of the clean reared survivorship curve toward the metal stressed curve resulting in $\mathrm{LT}_{50}$ values that are not significantly different. The alternative 
scenario is that the genes responsible for $\mathrm{Cu}^{2+}$ metabolism had already been up regulated at the time of the acute toxicity assay as a result of previous exposure. Metal detoxification is controlled to a large extent by the zinc finger transcription factor, metal response element-binding transcription factor-1 (MTF-1) (Balamurugan, et al., 2004). A major target gene of MTF-1 is metallothionein. In addition, glutathione $S$-transferases confer tolerance to oxidative stress (Leiers et al., 2003) and cytochrome P450-dependent monooxygenases have been shown to be altered by exposure to metals (Poupardin et al., 2008). $\mathrm{Cu}^{2+}$ transporter (Ctr1) likely also plays a role by its down regulation (Turski and Thiele, 2007). Transcription for all of these genes could have been altered by preexposure to $\mathrm{Cu}^{2+}$ to give pre-exposed larvae an advantage in the toxicity assay, i.e., hormesis. Regardless of the mechanism, larvae reared under metal stress have the potential to tolerate higher subsequent metal stress.

\subsubsection{AaMtn expression and the midgut}

This present study has shown the potentially high transcription activity of the $A$. aegypti metallothionein gene ( $A a \mathrm{Mtn})$ to be dynamic, reversible and expressed mostly in the midgut thereby underscoring the role of the midgut as a defensive barrier. $A a \mathrm{Mtn}$ is dramatically up regulated in response to $\mathrm{Cu}^{2+}$ in first instar $A$. aegypti, a critical life stage where most mortality in $\mathrm{Cu}^{2+}$ reared larvae occurs. The insect midgut is recognized as a barrier to parasites, xenobiotics and pathogens via the peritrophic matrix, a protective lining of the midgut (Beaty, et al., 2002). Intestinal mucin and alpha tubulin are constituent proteins of the peritrophic matrix and these genes have been shown to respond to metal exposure. This investigation, however, is the first report of 
metallothionein (Mtn) expression in the midgut of mosquitoes demonstrating a tissue specific physiological role for this protein in a disease vector. This work has demonstrated that $A a \mathrm{Mtn}$ is highly inducible by $\mathrm{Cu}^{2+}$ and that $A a \mathrm{Mtn}$ expression in body tissues (without midgut) of both clean reared and metal stressed treatments were virtually the same suggesting that $\mathrm{Cu}^{2+}$ did not go beyond the midgut tissue. These results suggest that the midgut is the primary tissue of $A a \mathrm{Mtn}$ expression and acts as a protective barrier for metal stress and are in agreement with the findings of Egli et al., who demonstrated in Drosophila melanogaster, that metallothioneins are expressed in a tissue-specific manner in response to $\mathrm{Cu}^{2+}$, i.e., the midgut (Egli, et al., 2006). Taken together, the dynamic and reversible nature of the Mtn gene along with its tissue specificity might explain why it has sometimes been problematic to use as a biomarker of environmental contamination.

Coping with metal stress comes at a cost however. Several studies have shown that metal stress decreases lipid reserves, increases respiration rate or alters the availability of energy yielding substrates (Servia, et al., 2006). Under the experimental conditions of this work (starvation), larvae exposed to chronic sub-lethal $\mathrm{Cu}^{2+}$ utilized more neutral storage lipids than larvae not exposed to $\mathrm{Cu}^{2+}$. These results are in agreement with other investigators and suggest that metal tolerance physiology is a lipid dependent process with neutral storage lipids fueling AaMtn expression.

\subsubsection{Implications for Vector Capacity}

Aedes aegypti exposed to urban metal pollution are likely at higher risk of increased vector capacity than their sylvatic counterparts. Adaptations conferring tolerance to metals are often costly to the organism's fitness and performance (Sakar, et 
al., 2004; Shirley and Sibly, 1999). Metal resistant strains of mosquitoes exhibit decreased egg viability, increased larval mortality, decreased pupation, emergence and adult survivorship when compared to non-selected strains (Mireji et al., 2010). It has also been shown that insecticide tolerant strains of anopheline and aedine mosquitoes exhibit increased fitness costs (Hemingway and Ranson, 2000). Our results demonstrating the compromised performance of adult female mosquitoes as a consequence of larval metal stress reinforce these and other previous works (Alto and Lounibus, 2012). Three mechanisms have been proposed that might lead to such fitness costs: a) detoxification mechanisms might use energy and nutritional resources that are then unavailable for other fitness traits such as growth or reproduction (Servia et al., 2006; Sorvari, et al., 2007, b) tolerant individuals might be less efficient at metal uptake or utilization resulting in micronutrient deficiency in unpolluted environments and c) oxidative stress may alter gene regulatory mechanisms involving redox signaling (Kitamura and Hiramatsu, 2010). Overall, these results suggest that metal stress, even at relatively low ecologically relevant concentrations, at the larval stage might alter adult mosquito performance and susceptibility to viral infection in much the same manner that larval stress increases vector competence as described by Alto and Lounibous (Alto and Lounibus, 2012 and references therein). Briefly, these authors demonstrated repeatedly that larval stress in the form of heat stress, competition, insecticide exposure and nutrient limitation each caused alterations in adult mosquito phenotype and immunity that increased their susceptibility to arboviruses. Larval metal stress is likely to have the same effect.

Many questions remain unresolved regarding the evolutionary fitness costs and vector capacity of insects that are tolerant to pollutants. The effect of insecticide 
resistance on vector capacity has been extensively investigated, but not the effect of environmental contaminants. The knowledge acquired from this body of work can be used to predict changes in the disease transmission dynamics of urban populations of mosquitoes; subsequently vector control agencies can make better informed decisions regarding vector control strategies.

\subsection{Acknowledgements}

We would like to thank Drs. Marcela Nouzova and Crisalejandra Rivera, and Elizabeth Leblanc and Stefano Correa for the critical reading and feedback of the

manuscript. This work was supported by NIH/NIG-MS R25 GM061347 and the FIU MBRS RISE Biomedical Research Initiative to MHP. 

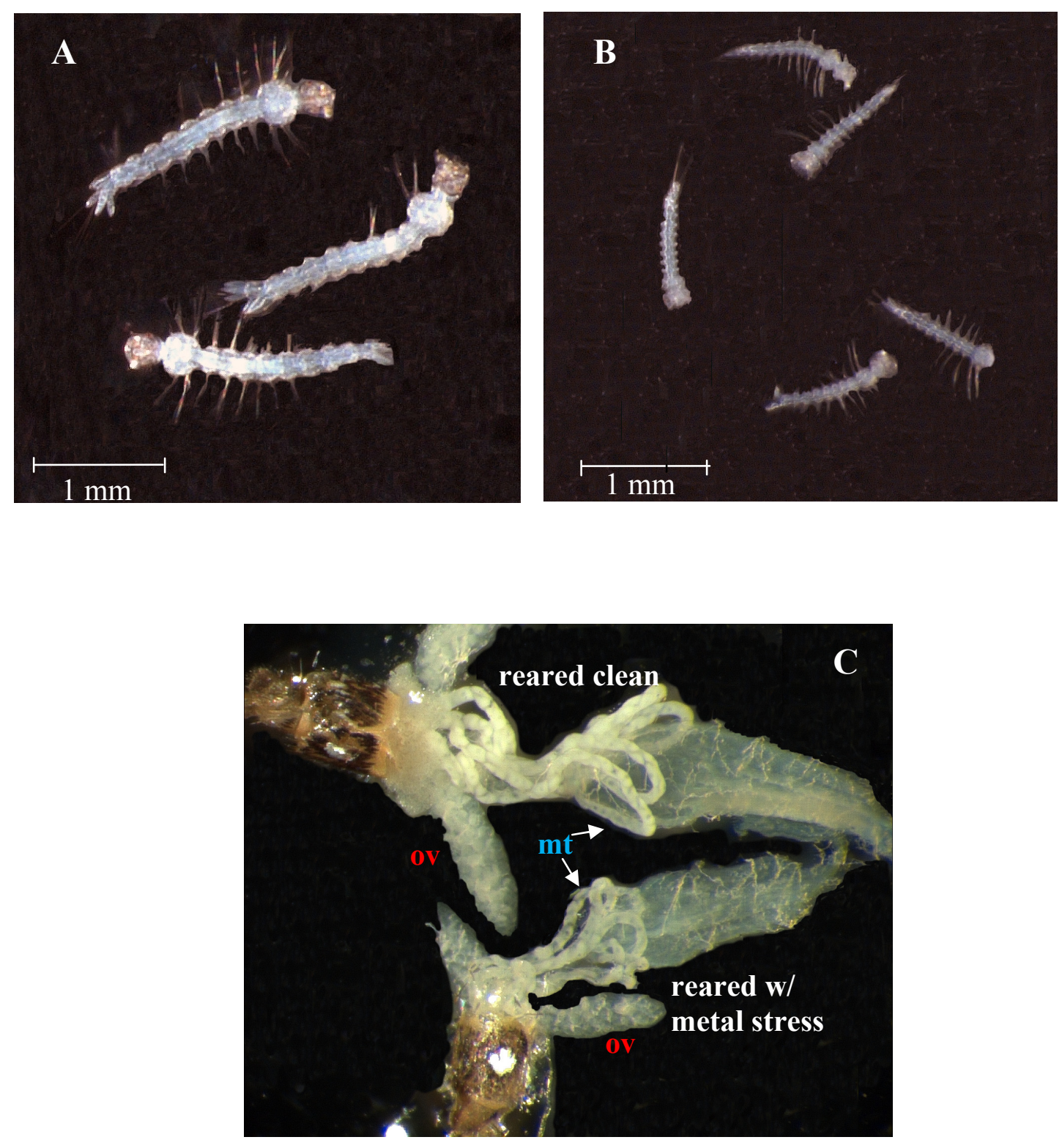

Fig. 4. Low sub-lethal metal stress compromised larval and adult phenotypes: Larvae reared in $1 \mathrm{ppm} \mathrm{Cu}^{2+}$ (B) are easily distinguish able after $24 \mathrm{~h}$ from those reared in clean water (A). Ovaries (ov) and malpighian tubules (mt) dissected from $3 \mathrm{~d}$ old adults were also compromised as a result of low sub-lethal larval metal exposure (C). Metal stressed larvae and adult tissues were less robust, pale and translucent in appearance. (all images40x magnification) 


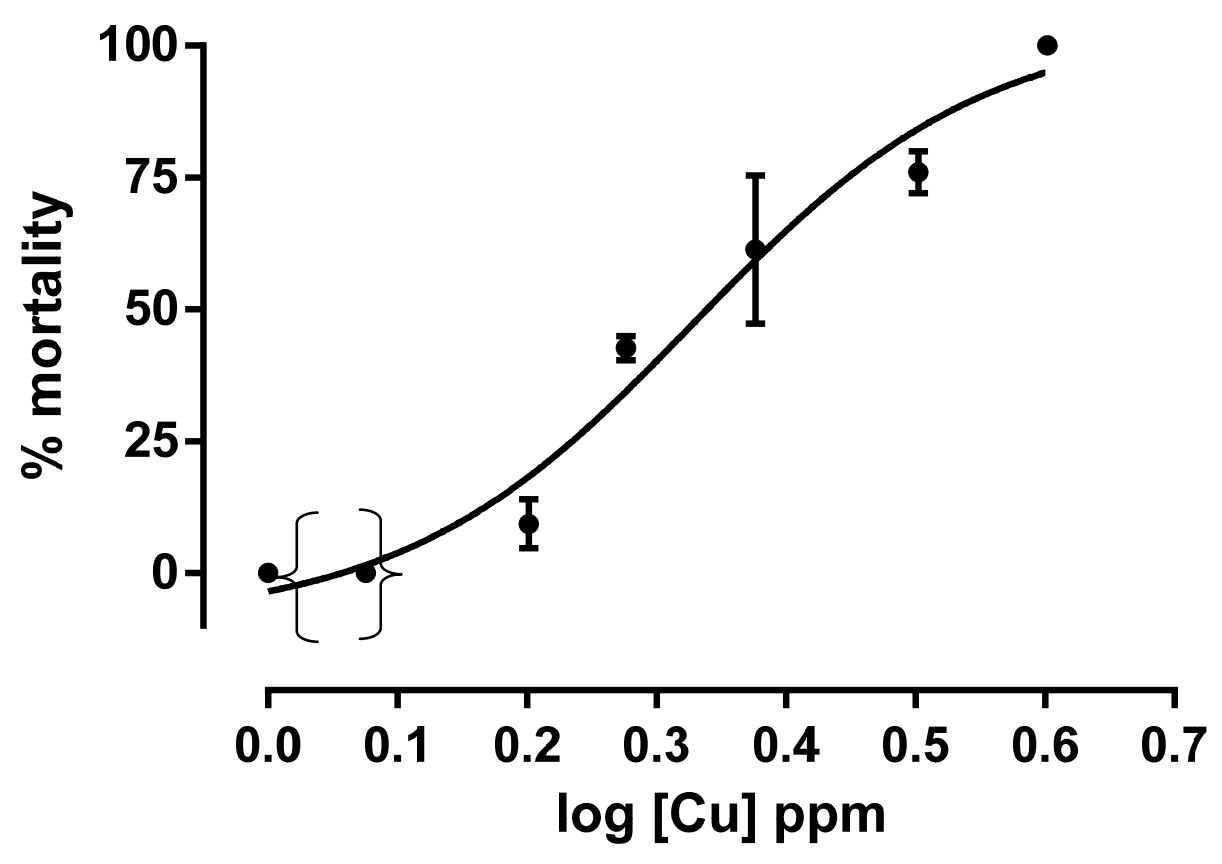

Fig. 5. Dose response curve for copper: A. aegypti $3^{\text {rd }}$ instars responded to $\mathrm{Cu}^{2+}$ in a dose dependent manner in a $24 \mathrm{~h}$ acute toxicity assay $\left(\mathrm{LOEC}=1.6, \mathrm{LC}_{50}=2.06 \mathrm{ppm}\right)$, $\left(\mathrm{n}=25\right.$, assayed in triplicate) (Sigmoidal nonlinear curve fit, $\left.\mathrm{r}^{2}=0.95\right)$. Brackets indicate concentrations at which mosquito larvae were reared for subsequent experiments throughout this work. 


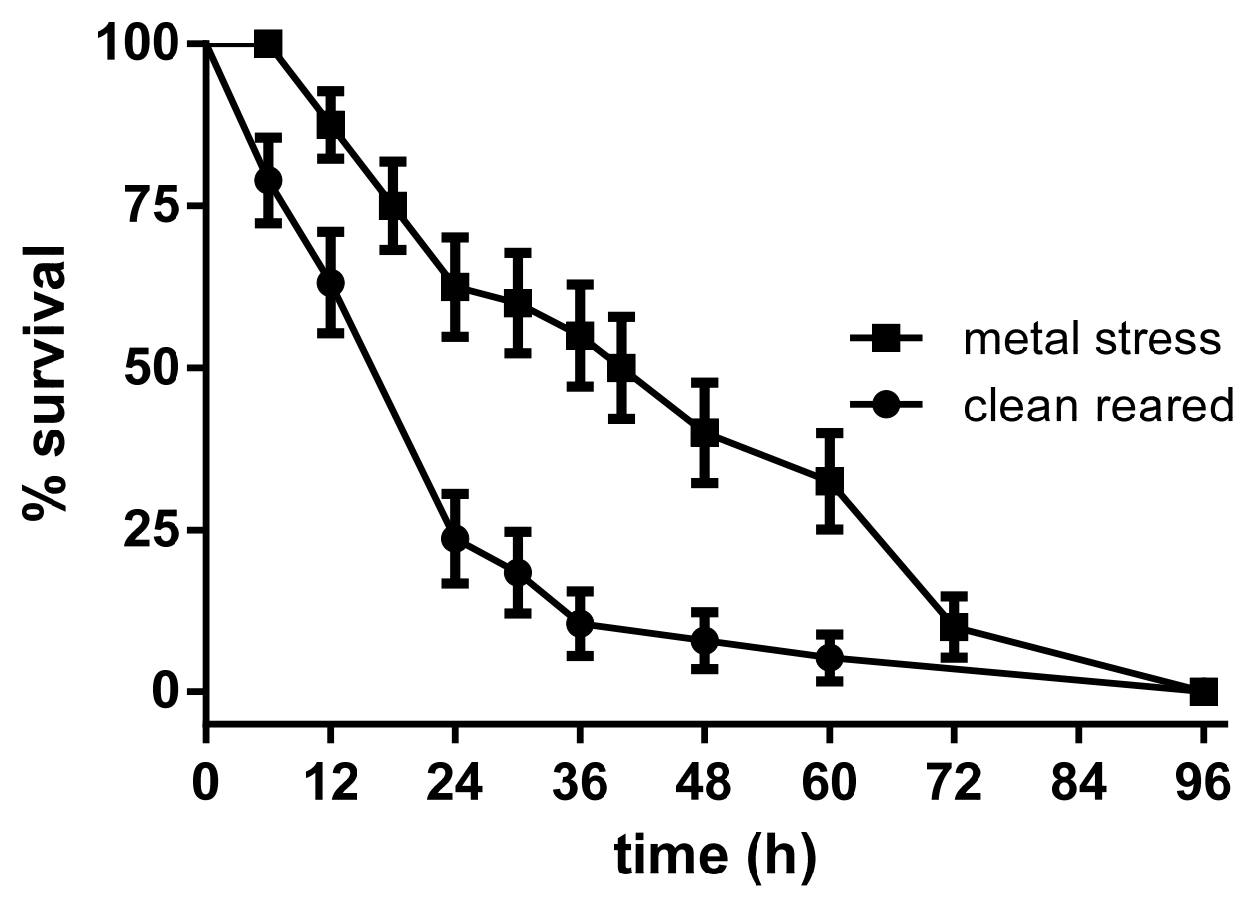

Fig. 6. Aedes aegypti $3^{\text {rd }}$ instars exhibited tolerance: Larvae reared in $0.75 \mathrm{ppm} \mathrm{Cu}^{2+}$ to the $3^{\text {rd }}$ instar stage exhibited significantly longer survivorship (median $\mathrm{LT}_{50}, 44$ vs. $14 \mathrm{~h}$ ) in an acute toxicity assay $\left(2.0 \mathrm{ppm} \mathrm{Cu}^{2+}\right)$ than larvae reared in clean water $(\mathrm{n}=20$ performed in duplicate, $\log$ rank test, Chi square $=4.65, \mathrm{p}=0.03$ ). 


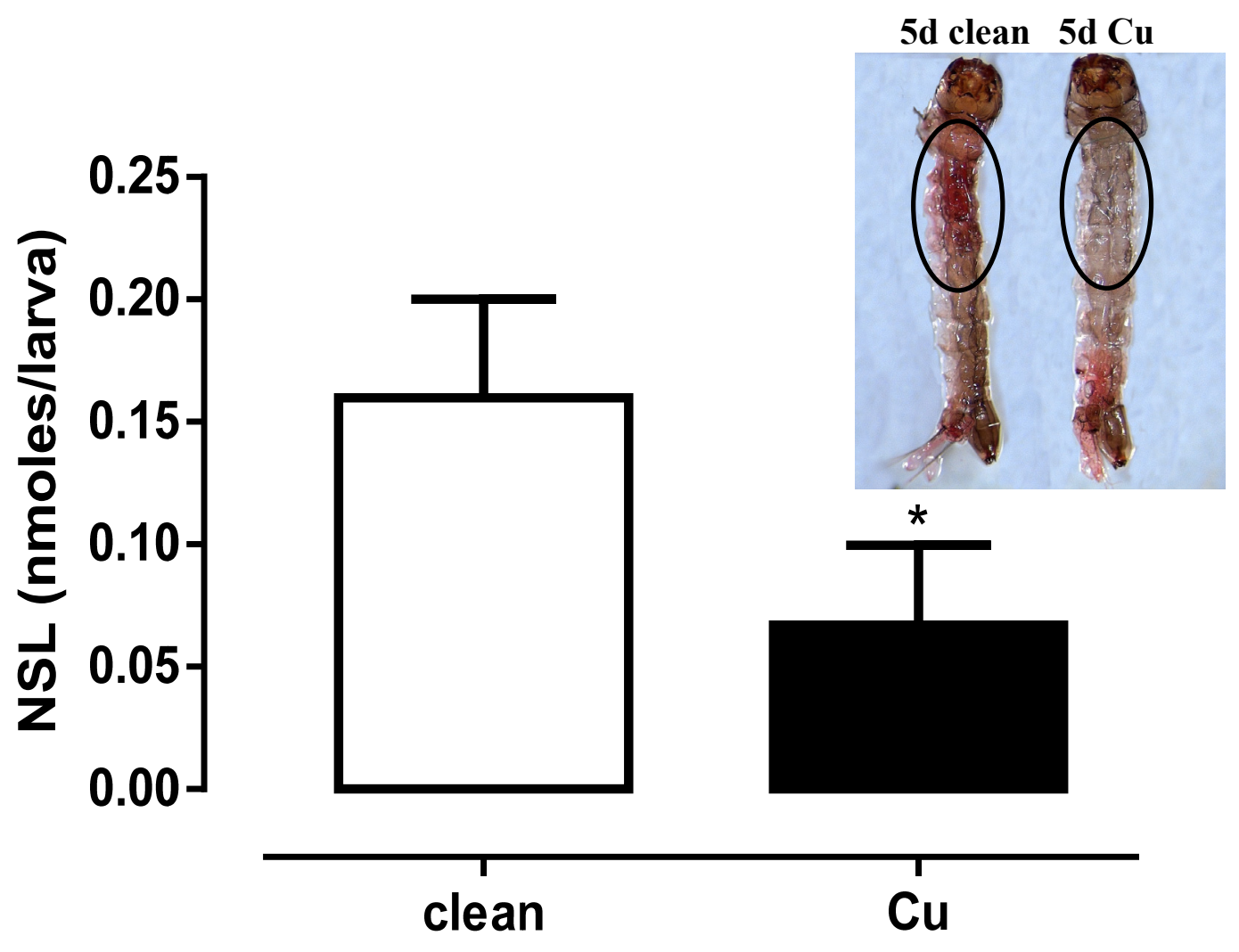

Fig. 7. The nutritional cost of metal stress: Fourth instar larvae held in $1.25 \mathrm{ppm} \mathrm{Cu}^{2+}$ for $5 \mathrm{~d}$ resulted in a reduction in neutral lipid reserves (NSL) compared to larvae held in clean water $(\mathrm{P}=0.018$, students $\mathrm{t}$ test, one tailed $)(\mathrm{n}=3$ assayed in triplicate). Representative image of fourth instar larvae stained with Oil Red O; ovals indicate area where fat body is located (inset). 

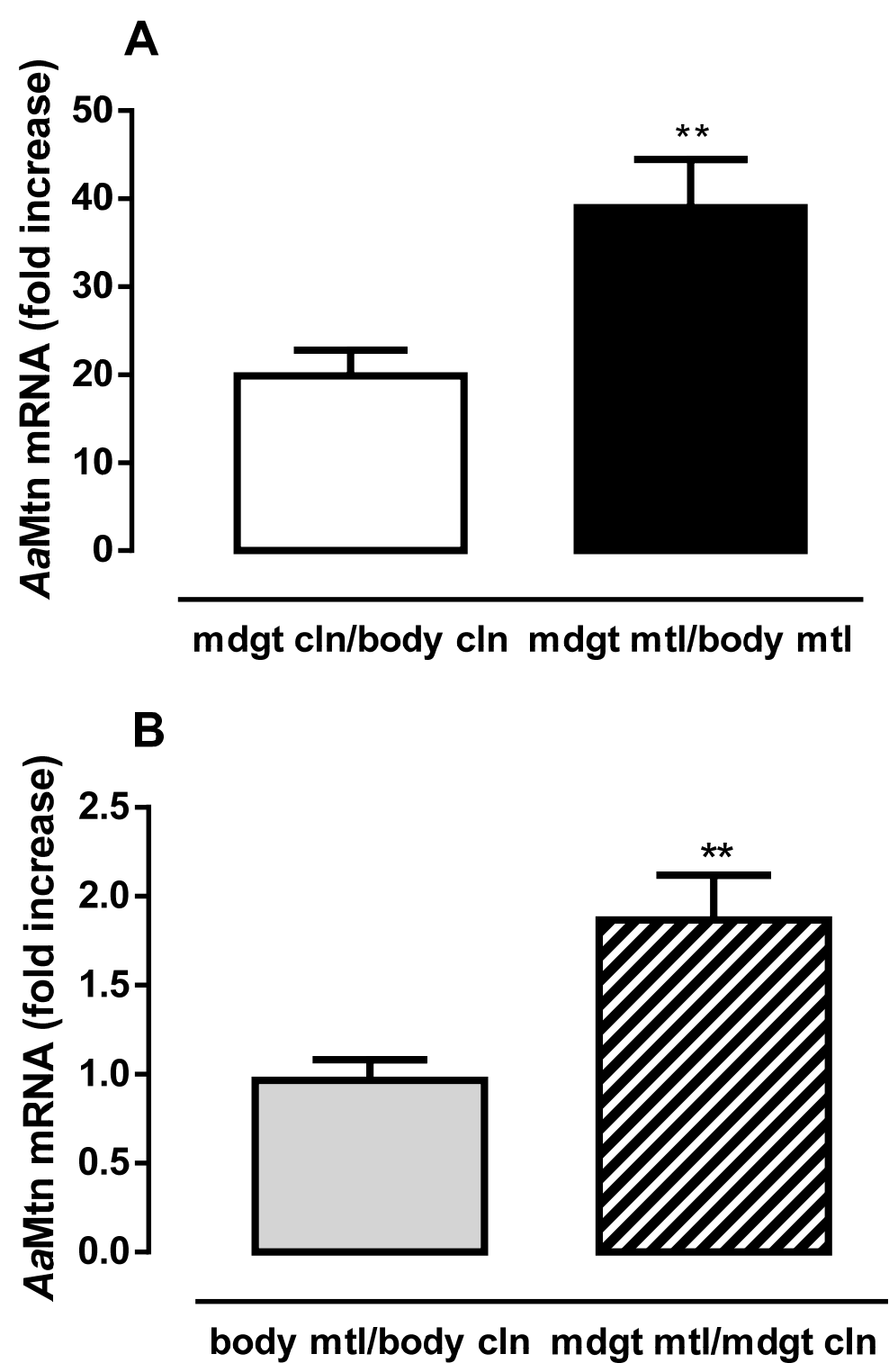

Fig. 8. AaMtn was expressed predominately in the midgut of larvae: (A) AaMtn expression in midguts (mdgt) of 4th instars held in clean water and exposed to $1.25 \mathrm{ppm}$ $\mathrm{Cu}^{2+}$ for $6 \mathrm{~h}$ was higher than AaMtn expression in the rest of the body. (B) Midguts of larvae exposed to metal stress expressed $\sim 2$ the AaMtn than midguts of larvae held in clean water. $A a \mathrm{Mtn}$ expression in body tissues of metal exposed and clean exposed were the same. ( $\mathrm{t}$ test, one way, $\mathrm{p}<0.01, \mathrm{df}=8$ ) (Three independent biological replicates of five tissues each $)($ mdgt $=$ midgut; body $=$ rest of the body minus midgut; cln = clean reared; $\mathrm{mtl}=$ metal stress) 


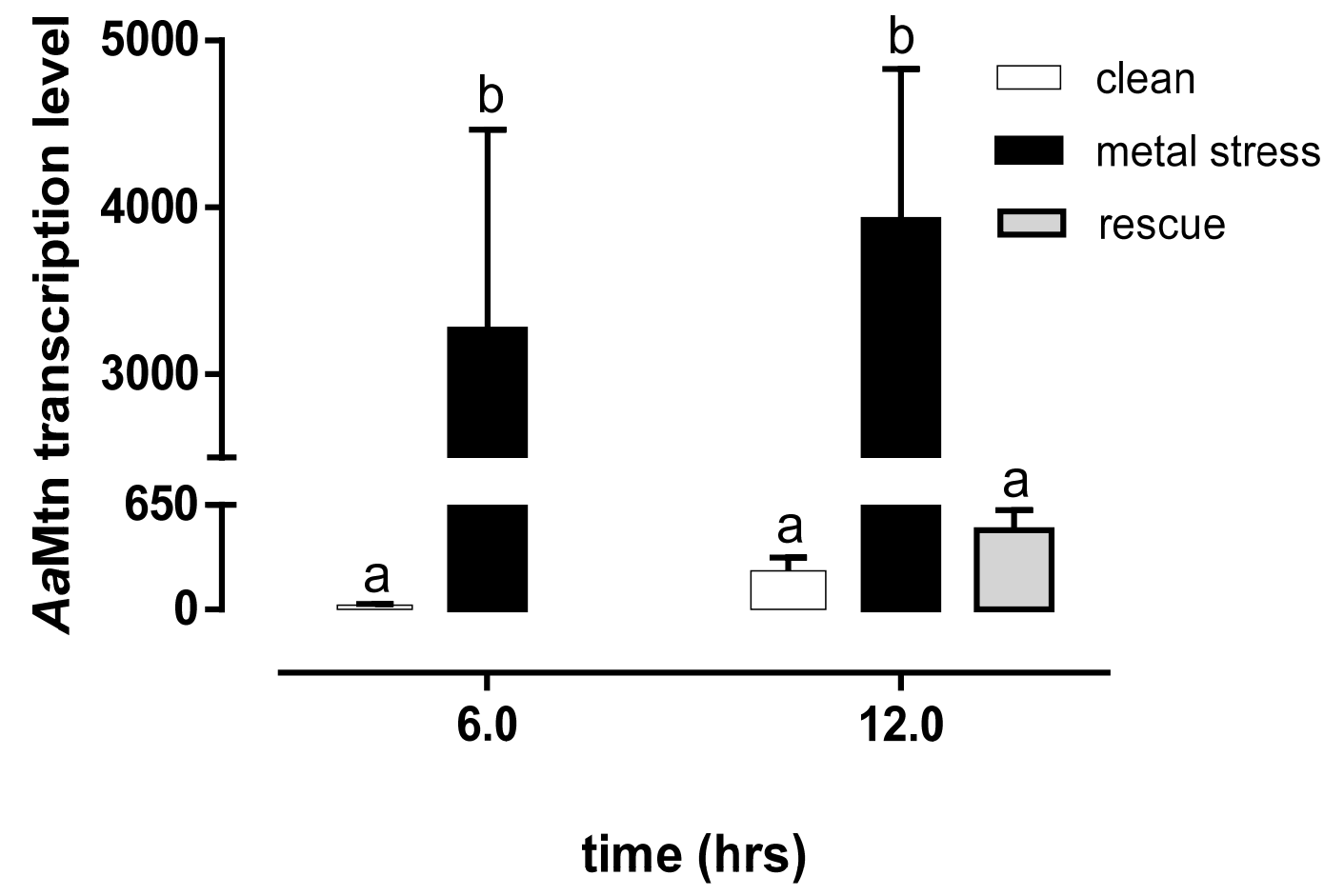

Fig. 9. AaMtn transcription is highly dynamic: Newly emerged first instars were exposed to $1.25 \mathrm{ppm} \mathrm{Cu}^{2+}$ for $12 \mathrm{~h}$ or held in clean water. For the rescue effect, larvae that were metal exposed to $\mathrm{Cu}^{2+}$ for $6 \mathrm{~h}$ were transferred back to clean water and held for $6 \mathrm{~h}$. $A a \mathrm{Mtn}$ mRNA is expressed as copy number of $A a \mathrm{Mtn}$ mRNA/ 10,000 copies of rpL32 mRNA. Each q-PCR data point is a mean \pm SEM of three independent biological replicates of $\sim 50$ larvae (one way ANOVA with a Tukey's multiple comparison test, $\mathrm{F}=7.02,19$ total $\mathrm{DF}, \mathrm{P}=0.0022$ ). Letter designations indicate significant differences for multiple comparison tests $(\mathrm{p}<0.05)$. 

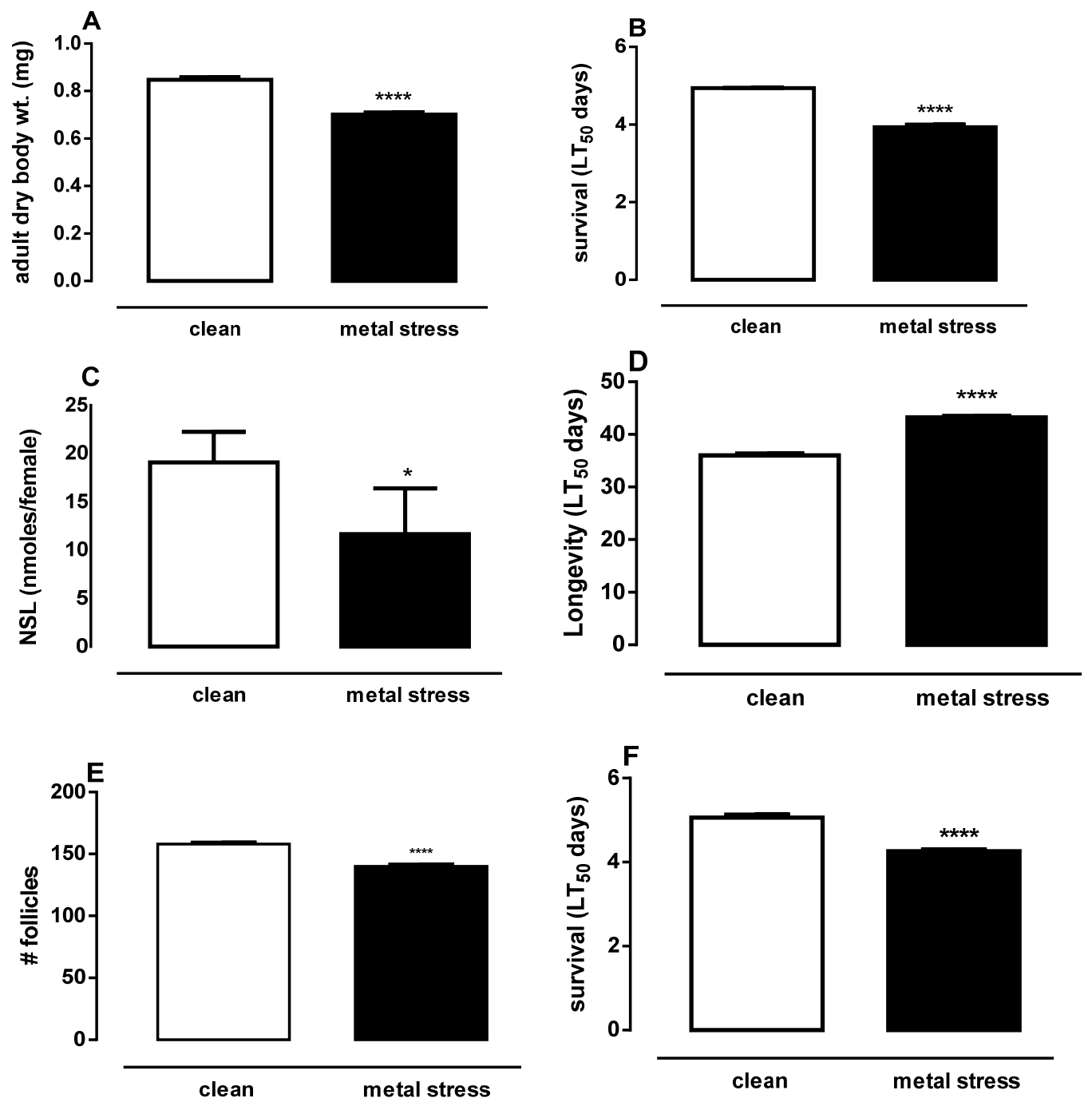

Fig. 10. Larval metal stress is reflected in adult phenotype: Larvae were reared in $1 \mathrm{ppm}$ $\mathrm{Cu}^{2+}$ or in clean water. (A) Larval metal stress resulted in smaller female adults $(\mathrm{n}=50$ 69) ( $t$ test, one tailed, $p<0.0001, t=10.72 \mathrm{df}=117$ ). (B) Starvation tolerance of newly emerged adult females was reduced by the larval metal stress $(n=15$, assayed in triplicate; log rank, $\mathrm{p}<0.0001$ ). (C) Newly emerged females reared under metal stress emerged with less neutral storage lipids (NSL) $(n=3$ assayed in triplicate; $p<0.04$, $t$ test, one tailed, $\mathrm{t}=2.252$, $\mathrm{df}=4$ ). (D) Adult females reared from metal stressed larvae exhibited longer lifespan ( $n=15$, assayed in triplicate; log rank, $p<0.0001)$. (E) Larval metal stress resulted in females developing fewer follicles $(n=15$ pairs of ovaries; $\mathrm{p}<0.0001, \mathrm{t}$ test, one tailed, $\mathrm{t}=40.38 \mathrm{df}=28$ ). (F) Larval metal stress resulted maternal effects i.e., lower starvation tolerance of first instar offspring (log rank, $\mathrm{p}<0.0001$ ). 


\subsection{References}

Alto, B. W. and L. P. Lounibos, Eds. (2012). Vector competence for arboviruses in relation to the larval environment of mosquitoes, Wageningen Academic Pub.

Amiard, J. C., C. Amiard-Triquet, et al. (2006). "Metallothioneins in aquatic invertebrates: Their role in metal detoxification and their use as biomarkers." Aquatic Toxicology 76(2): 160-202.

Balamurugan, K., D. Egli, et al. (2007). "Copper homeostasis in Drosophila by complex interplay of import, storage and behavioral avoidance." EMBO 26(4): 1035-1044.

Balamurugan, K., D. Egli, et al. (2004). "Metal-responsive transcription factor (MTF-1) and heavy metal stress response in Drosophila and mammalian cells: a functional comparison." Biol Chem 385: 597-603.

Barrera, R., M. Amador, et al. (2008). Unusual productivity of Aedes aegypti in septic tanks and its implications for dengue control. Medical and Veterinary Entomology. 22: 62-69.

Beaty, B. J., R. S. Mackie, et al. (2002). "The midgut epithelium of aquatic arthropods: A critical target organ in environmental toxicology." Environmental Health Perspectives 110: 911-914.

Crovello, T. J. and C. S. Hacker (1972). "Evolutionary Strategies in Life Table Characteristics Among Feral and Urban Strains of Aedes aegypti (L.)." Evolution 26(2): 185-196.

Egli, D., H. Yepiskoposyan, et al. (2006). "A family knockout of all four Drosophila metallothioneins reveals a central role in copper homeostasis and detoxification." Molecular and Cellular Biology 26(6): 2286-2296.

Frankie, G. W. and L. E. Ehler (1978). "Ecology of Insects in Urban Environments." Annual Review of Entomology 23(1): 367-387.

Gubler, D. J. (2004). "The changing epidemiology of yellow fever and dengue, 1900 to 2003: full circle?" Comparative Immunology, Microbiology and Infectious Diseases 27(5): 319-330.

Hemingway, J., Ranson, Hilary (2000). "Insecticide Resistance in Insect Vectors of Human Disease " Annu. Rev. Entomol. 45 371-391.

Jansen, C. C. and N. W. Beebe (2010). "The dengue vector Aedes aegypti: what comes next." Microbes and Infection 12(4): 272-279. 
Kitamura, M. and N. Hiramatsu (2010). "The oxidative stress: endoplasmic reticulum stress axis in cadmium toxicity." Biometals 23(5): 941-950.

Kosalwat, P. and A. W. Knight (1987). "Acute toxicity of aqueous and substrate-bound copper to the midge, Chironomus decorus." Archives of Environmental Contamination and Toxicology 16(3): 275-282.

Kosalwat, P. and A. W. Knight (1987). "Chronic toxicity of copper to a partial life cycle of the midge,Chironomus decorus." Archives of Environmental Contamination and Toxicology 16(3): 283-290.

Leiers, B., A. Kampkötter, et al. (2003). "A stress-responsive glutathione S-transferase confers resistance to oxidative stress in Caenorhabditis elegans." Free Radical Biology and Medicine 34(11): 1405-1415.

Marinkovic, M., R. A. Verweij, et al. (2011). "Life Cycle Responses of the Midge Chironomus riparius to Compounds with Different Modes of Action." Environmental Science \& Technology 45(4): 1645-1651.

Mireji, P. O., J. Keating, et al. (2010). "Biological cost of tolerance to heavy metals in the mosquito Anopheles gambiae." Medical and Veterinary Entomology 24(1).

Mireji, P. O., J. Keating, et al. (2008). "Heavy metals in mosquito larval habitats in urban Kisumu and Malindi, Kenya, and their impact." Ecotoxicology and Environmental Safety 70(1): 147-153.

Montgomery, M. R. (2008). The Urban Transformation of the Developing World. Science. 319: 761-764.

Newman, M. C. and M. A. Unger, Eds. (2002). Fundaments of Ecotoxicolgy Lewis Publishers.

Perez, M. H. and F. G. Noriega (2012). "Aedes aegypti pharate first instar quiescence affects larval fitness and metal tolerance." Journal of Insect Physiology 58(6): 824-829.

Posthuma, L. and N. M. Van Straalen (1993). "Heavy-metal adaptation in terrestrial invertebrates: a review of occurrence, genetics, physiology and ecological consequences." Comparative biochemistry and physiology. C. Comparative pharmacology and toxicology 106(1): 11-38 
Poupardin, R., S. Reynaud, et al. (2008). "Cross-induction of detoxification genes by environmental xenobiotics and insecticides in the mosquito Aedes aegypti: Impact on larval tolerance to chemical insecticides." Insect Biochemistry and Molecular Biology 38(5): 540-551.

Puig, S. and D. J. Thiele (2002). "Molecular mechanisms of copper uptake and distribution." Current Opinion in Chemical Biology 6(2): 171-180.

Rayms-Keller, A., K. E. Olson, et al. (1998). "Effect of Heavy Metals on Aedes aegypti (Diptera:Culicidae) Larvae." Ecotoxicology and Environmental Safety 39(1): 4147.

Sarkar, S., A. K. Duttagupta, et al. (2004). "Effects of heavy metals on population growth and metallothionein gene expression in the mosquito Culex quinquefasciatus, from Calcutta, India." Environmental Pollution 127(2): 183193.

Servia, M., A. Péry, et al. (2006). "Effects of copper on energy metabolism and larval development in the midge Chironomus riparius." Ecotoxicology 15(3): 229-240.

Shirley, M. D. F. and R. M. Sibly (1999). "Genetic Basis of a between-Environment Trade-off Involving Resistance to Cadmium in Drosophila melanogaster." Evolution 53(3): 826-836.

Sorvari, J., L. M. Rantala, et al. (2007). "Heavy metal pollution disturbs immune response in wild ant populations." Environmental Pollution 145(1): 324-328.

Turski, M. L. and D. J. Thiele (2007). "Drosophila Ctr1A Functions as a Copper Transporter Essential for Development." Journal of Biological Chemistry 282(33): 24017-24026. 


\section{Chapter 4: Aedes aegypti pharate first instar quiescence affects larval fitness and metal tolerance}

\subsection{Abstract}

The eggs of the mosquito, Aedes aegypti, possess the ability to undergo an extended quiescence hosting a fully developed first instar larvae within the chorion. As a result of this life history trait, pharate larvae can withstand months of quiescence inside the egg where they depend on stored maternal reserves. Aedes aegypti mosquitoes are frequently associated with urban habitats that may contain significant metal pollution. Therefore, the duration of quiescence and extent of nutritional depletion may affect the physiology and survival of larvae that hatch in a suboptimal habitat. The aim of this study was to determine the effect of an extended quiescence on larval nutrient reserves and the subsequent effects of metal exposure on larval fitness, survival and development. It is hypothesized that an extended quiescence would reduce nutritional reserves and would alter the molecular response to metal exposure thereby reducing larval survival and altering larval development. As a molecular marker for metal stress responses, we evaluated transcriptional changes in the metallothionein gene $(A a \mathrm{Mtn})$ in response to quiescence and metal exposure.

Extended first instar quiescence resulted in a significant decrease in lipid reserves and negatively affected larval fitness and growth. AaMtn transcription and metal tolerance were compromised in first instars emerged from eggs that had undergone an extended quiescence. These findings suggest that newly emerged mosquito larvae that had survived a relatively long pharate first instar quiescence (as might occur during a dry 
season) are more vulnerable to environmental stress. Pharate first instar quiescence could have implications for vector control strategies. Newly emerged mosquito larvae at the end of the dry season or start of the wet season are physiologically compromised, and therefore potentially more susceptible to vector control strategies than mosquito larvae hatched subsequently throughout the wet season.

\subsection{Introduction}

Container breeding mosquitoes such as Aedes aegypti possess the ability to undergo aseasonal quiescence as an unhatched first instar. Embryonic development is completed within $\sim 3$ days after oviposition and a fully developed first instar larva resides within the chorion awaiting the appropriate environmental cues to stimulate hatching (Clements, 1992). This unique quiescent stage does not have a clear homologue in other insects and has been referred in previous work as a pharate first instar diapause (Alekseev, 2007; Clements, 1992). To be a true diapause, the arrest should persist for some period of time even if the right environmental conditions are present; therefore we will refer to this stage as pharate first instar quiescence. As a result of this life history trait, Aedes aegypti produce eggs that in addition to being desiccation resistant, can withstand months of dormancy. Despite the obvious advantages of aseasonal quiescence, the energetic costs of long-term dormancy are reflected in lower post-hatching survival and reduced fecundity (Hahn and Denlinger, 2007).

Aedes aegypti, the primary vector of dengue fever, is well adapted to survive and develop in the anthropogenic bodies of water characteristic of many urban environments (Crovello and Hacker, 1972; Huber, 2008; Trpis and Hausermann, 1975). These breeding 
sites can be characterized by heterogeneous and dynamic conditions such as varying water volume and/or nutrient content and the presence of toxic metals and/or other physiological stressors. Metals in particular, are common in urban environments, where there exist many sources of metal pollution such as run-off, atmospheric fall out, and the indiscriminant use of metal-containing herbicides. (Mireji, et al., 2008; Sarkar et al., 2004). In addition, the breeding sites themselves may be metallic (cans, dumpsters, cisterns, etc.). The ability to adjust to metal-polluted urban environments may provide distinct advantages to larval mosquitoes. However, larval mosquitoes may also incur fitness costs. Previous studies suggested that metal stress during mosquito larval development might affect mosquito's fitness and ability to transmit diseases (RaymsKeller, 1998; Sarkar et al., 2004; Mireji et al., 2010a).

Although excess metal ions in the aquatic environment are generally toxic, mechanisms exist in larval mosquitoes to chelate, sequester or otherwise eliminate or detoxify such toxins. One such mechanism is the expression of metallothioneins (Mtn). Metallothioneins are a group of low molecular weight cysteine-rich proteins essential for metal homeostasis, sequestration and detoxification; they have a high affinity for transition metals and are expressed in response to metal exposure in a wide variety of organisms (Amiard, et al., 2006; Andrews, 2000; Balamurugan, et al., 2007; Egli, et al., 2006; Kagi, 1991; Postuma, and van Straalen, 1993; Roesijadi, 1996). Mtn transcription has been validated as a sensitive indicator of metal exposure and an appropriate molecular marker to assess the physiological response to metal stress (Dallinger,1994; Egli, et al., 2006; Mireji, et al., 2010b; Sarkar, et al., 2004). 
The aim of this study was to determine if the duration of pharate first instar quiescence (newly oviposited eggs $v s$. eggs having undergone an extended quiescence period) would reduce the ability of newly hatched larvae to tolerate metal stress. To test this hypothesis the effect of quiescence on the physiological and molecular responses to metal stress in newly hatched larval mosquitoes was assessed in three ways: 1) by determining the duration of the development period, tolerance to starvation and quantifying neutral lipids in first instar larvae hatched from new and old eggs, 2) by characterizing the response of larvae hatched from new or old eggs to acute and chronic

exposures to copper $\left(\mathrm{Cu}^{2+}\right)$ and 3) by describing the molecular response to metal exposure through quantification of metallothionein mRNA's in larvae hatched from new or old eggs exposed to $\mathrm{Cu}^{2+}$. Extended pharate first instar quiescence results in a significant decrease in lipid reserves and negatively affects larval fitness and growth. In addition, metallothionein transcription and metal tolerance were compromised in first instars emerged from eggs that have been subjected to extended quiescence. These findings suggest that newly emerged mosquito larvae that had survived a long pharate first instar quiescence (as might occur during a dry season) are more vulnerable to environmental stress.

\subsection{Materials and Methods}

\subsubsection{Mosquito rearing and maintenance}

Aedes aegypti of the Rockefeller strain were reared in $28{ }^{\circ} \mathrm{C}$ and $80 \%$ relative humidity under a photoperiod of $16 \mathrm{~h}$ light: $8 \mathrm{~h}$ dark. Mated adults were offered a cotton pad soaked in 3\% sucrose solution. Four-day-old female mosquitoes were fed porcine 
blood equilibrated to $37^{\circ} \mathrm{C}$, and ATP was added to the blood meal to a final

concentration of $1 \mathrm{mM}$ immediately before use. Oviposited eggs were collected after $96 \mathrm{~h}$ and stored under insectary conditions on egg papers in zip lock bags in Tupperware containers (adapted from Munstermann, 1997). Biological replication was achieved by generating eggs and or larvae as needed from independent and separate hatches of mosquito eggs across numerous generations.

\subsubsection{Reagents}

Reconstituted soft water (RSW) was used in all bioassays. RSW was prepared by adding reagent-grade $\mathrm{NaHCO}_{3}(48 \mathrm{mg}), \mathrm{CaSO}_{4} 2 \mathrm{H}_{2} \mathrm{O}(30 \mathrm{mg}), \mathrm{MgSO}_{4}(30 \mathrm{mg})$ and $\mathrm{KCl}$ (2mg) to 1 liter of deionized water ( $\mathrm{pH}$ 7.4). All reagents were purchased from Fisher Scientific, Pittsburgh, PA.

\subsubsection{Copper measurement}

Reagent grade copper sulfate pentahydrate was dissolved in RSW. Nominal $\mathrm{Cu}^{2+}$ concentrations were confirmed by inductively coupled plasma mass spectrometric analysis (ICP-MS) using an HP 4500 plus IPC-MS instrument (Hewlett-Packard Co., Wilmington, DE), equipped with a Babington-type nebulizer and an ASX-500 autosampler (Cetac Technologies Inc., Omaha, NE).

\subsubsection{Fitness assays}

In the manuscript, "new eggs" are pharate first instars that remained dormant for less than one week. "Old eggs" are pharate first instars that have undergone extended 
quiescence for over two months. Experiments were performed under the temperature, humidity and light cycle conditions described in section 2.1 ("Insectary conditions"). All assays were started at $\sim 12: 00 \mathrm{pm}$.

a) Time to pupation (duration of development period): Pharate first instars (eggs) were hatched in deoxygenated water. Upon emergence, 200 first instar larvae were reared to adulthood in $23 \times 40 \times 15 \mathrm{~cm}$ polypropylene plastic pans containing $1 \mathrm{~L}$ of $\mathrm{RSW}$ for the reared clean treatment and $1 \mathrm{ppm} \mathrm{Cu}^{2+}$ for the metal stressed treatment. Larvae reared in copper $(\mathrm{Cu})$ are referred to as "metal stressed". Larval diet consisted of $750 \mu 1$ of $10 \%$ liver powder suspension (MP Biomedicals Inc., Aurora, OH.) added to the pan every day except day two. Pupae were counted each day (at noon) throughout the pupation period. The time to pupation was calculated using the following equation: $\mathrm{T}=\sum(\mathrm{DxN}) / \sum(\mathrm{N})$ (Kosalwat and Knight, 1987), where: $\mathrm{T}=$ the average time to pupation (days), $\mathrm{D}=$ number of days from hatching to pupation (age in days) and $\mathrm{N}=$ number of insects pupated at that day.

b) Larval survival under starvation conditions: Newly emerged first instar larvae from new and old eggs were assayed in $15 \mathrm{~cm}$ diameter glass petri dishes containing $100 \mathrm{ml}$ of RSW in the absence of food. The number of larvae found not responsive to mechanical stimulation every $24 \mathrm{~h}$ were counted as "dead" and removed.

\subsubsection{Acute toxicity stress assays}

Newly emerged first instar larvae from new and old eggs were assayed in $15 \mathrm{~cm}$ diameter glass petri dishes containing $100 \mathrm{ml}$ of RSW containing $1.25 \mathrm{ppm}$ of $\mathrm{Cu}$ (from $\mathrm{CuSO}_{4}$ ) for $48 \mathrm{~h}$. A concentration of $1.25 \mathrm{ppm} \mathrm{Cu}^{2+}$ was chosen based on preliminary $24 \mathrm{~h}$ 
acute toxicity assays $\left(\sim \mathrm{LC}_{30}\right)$. Experiments were performed under insectary conditions and in the presence of food ( $75 \mathrm{ul} 10 \%$ liver powder suspension). Larvae found not responsive were counted as "dead". Negative controls were reared in the same conditions in the absence of $\mathrm{Cu}^{2+}$.

\subsubsection{Lipid and protein analysis}

a) Staining of lipids: Newly emerged first instars hatched from new and old eggs were fixed in $4 \%$ formaldehyde for $2 \mathrm{~h}$, washed with cold PBS, stained with Oil Red-O for 30 min., washed with cold PBS and imaged with a Leica Model MZ6 stereomicroscope at 40x magnification (adapted from Nishiura et. al., 2007).

b) Quantification of total larval neutral lipids: Neutral lipids were quantified using a triglyceride quantification kit (Biovision, Mountain View, CA; cat\# K622-100). Ten newly emerged first instars from each treatment (new $v s$. old) were assayed in triplicate for three biological replicates.

c) Quantification of total larval proteins: Total protein contents of larvae were quantified using a Pierce $660 \mathrm{~nm}$ Protein Assay from Fisher Scientific (prod. \#322662). Fifty newly emerged first instars from each treatment (new $v s$. old) were assayed in triplicate for three biological replicates.

\subsubsection{Quantitative real-time PCR ( $q P C R)$}

Newly emerged first instar larvae from new and old eggs were exposed to 1.25 ppm of $\mathrm{Cu}^{2+}$ (from $\mathrm{CuSO}_{4}$ ) in RSW under insectary conditions and in the absence of food for $6 \mathrm{~h}$. Total RNA was isolated from $\sim 50$ larvae using RNA-binding glass powder 
as previously described (Noriega and Wells, 1993). Contaminating genomic DNA was removed using the DNA-free ${ }^{\mathrm{TM}}$ kit (Ambion, Austin, TX). Reverse transcription was carried out using the Verso cDNA Kit (Fisher Scientific). Real time qPCR was performed with the 7300 Real Time PCR System using TaqMan ${ }^{\circledR}$ Gene Expression Assays together with TaqMan ${ }^{\circledR}$ Universal PCR Master Mix (Applied Biosystems, Foster City, CA). The primer probes for housekeeping gene 60S ribosomal protein L32 (rpL32) (Accession number: AAEL003396) and for metallothionein (Mtn) (Accession number: AAEL008176) were as follows (exon borders in bold):

rpL32 Forward: 5' CCAAGCCAGATTCCTAACATTCAAA 3'

rpL32 Reverse: 5' TGCCCTGTCCGCAACC 3'

rpL32 Probe: 5' CTTGCAATCATTACCACAGCAC 3'

Mtn Forward: 5' GCCAAGCCAGATTCCTAACATTCAA 3'

Mtn Reverse: 5' GCAACCGGAGGTGCACTT 3'

Mtn Probe: 5' AagtgCtgtggtaAtgattgC 3'

qPCR reactions were carried in a $20 \mu \mathrm{l}$ volume according to manufacturer's recommendations for Custom TaqMan ${ }^{\circledR}$ Gene Expression Assays. Reactions were run in triplicate using $1 \mu \mathrm{l}$ of cDNA per reaction. Standard curves to quantify relative gene copy number were made from serial dilutions of plasmids containing rpL32 or Mtn gene $(300,000,30,000,3000,300$ and 30 copies of a plasmid per reaction). Real time data were collected by 7300 System SDS Software and analyzed in Microsoft Excel. Mtn transcript levels were normalized with rpL32 transcript levels in the same sample. Relative Mtn transcript levels are expressed as a number of copies of Mtn transcript per 
10,000 copies of rpL32 transcript. Each qPCR data point is average of three independent biological replicates.

\subsubsection{Data analysis}

Graphical representations were performed with GraphPad Prism Software (San Diego, CA) version 3.00 for Windows. Statistical analysis was performed with Excel 2007. The results were expressed as mean and SEM and considered significantly different at $P<$ 0.05 by a one tailed students $t$ test $(\mathrm{P}<0.05)$. Significant differences in variance were determined by F test.

\subsection{Results}

4.4.1 Effect of quiescence on first instar larval survivorship and growth

When reared in a clean environment, larvae hatched from older eggs exhibit a significantly longer larval development period than larvae hatched from new eggs as evidenced by a mean 7.33 vs. 5.95 days to pupation (Fig. 11). There was no observed mortality in either treatment.

\subsubsection{Effect of quiescence on larval nutrient reserves}

first instar larvae hatched from older eggs exhibited a significantly higher mean mortality in response to starvation (76 vs $4 \%$ ) in a $72 \mathrm{~h}$ acute assay (Fig. 12). Neutral lipid reserves decreased approximate nine fold in pharate first instars larvae that had been

subjected to three month quiescence (old eggs) (Fig. 13). Quiescence had no effect on the 
total amount of protein measured in newly hatched larvae. Both groups had approximately $150 \mathrm{ng}$ protein/larva $(\mathrm{n}=50)$.

\subsubsection{Effect of quiescence on first instar larvae response to metal stress}

Newly emerged first instar larvae hatched from old eggs exhibited a two-fold increase in mortality ( $70 \%$ vs. $35 \%$ in new eggs) in a $48 \mathrm{~h}$ acute toxicity assay (Fig. 14). When larvae are reared to adulthood in the presence of metal stress, larvae hatched from older eggs exhibited a significantly higher mortality than larvae emerged from new eggs ( $85 \%$ vs. 53\% respectively) and a significantly longer larval development period than larvae hatched from new eggs (15.02 vs. 17.38 days) (Fig.15).

\subsubsection{Effect of quiescence on metallothionein mRNA levels}

Newly emerged first instar larvae from new eggs exposed to $\mathrm{Cu}^{2+}$ for $6 \mathrm{~h}$ exhibited a 26 fold increase in $A a \mathrm{Mtn}$ mRNA levels, whereas newly emerged first instar larvae from old eggs exposed to $\mathrm{Cu}^{2+}$ for $6 \mathrm{~h}$ exhibited only a fourfold increase (Fig. 16). The Mtn response to metal stress of first instars hatched from old eggs was significantly less robust throughout the time course. In addition, AaMtn mRNA levels from first instars from old eggs did not exhibit the magnitude in variance that first instars from new eggs did. Variances in Mtn response are significantly different at all-time points except at $3 \mathrm{~h}(\mathrm{~F}$ test, $\mathrm{p}<0.05)$. There was a moderate but significant increase on Mtn mRNA levels in larvae held in clean RSW for $6 \mathrm{~h}$, regardless of the eggs' age. This increase is most likely related with the developmental and homeostatic roles of Mtn in mosquito larvae. 


\subsection{Discussion}

4.5.1 The duration of quiescence alters the nutritional status, growth rate and starvation tolerance of newly hatched larvae

It would seem there was a simple relationship between the duration of quiescence and the nutritional and physiological status of newly hatched larvae. First instars hatched from eggs subjected to an extended quiescence emerged with less lipid reserves, were less tolerant to starvation and had a longer larval development period. The fertilized mosquito egg is a closed system, bound by an impermeable chorion and dependent on maternally-derived lipid reserves to complete embryogenesis and maintain a quiescent metabolism. Over 90\% of stored egg lipids are triglycerides (van Handel, 1993) and are likely critical for energy production during quiescence in first instar larvae (Hahn and Denlinger, 2007). The observed reduction of lipids in larvae hatched from old eggs is probably the result of the oxidation of lipids to support basal metabolism (Hahn and Denlinger, 2007). Although oxygen consumption and metabolism are depressed after embryogenesis is completed, (Weissman-Strum and Kindler, 1962), an extended first instar larvae quiescence would eventually deplete egg reserves.

Larval growth depends on maternally inherited reserves in addition to nutrients acquired by the larvae. These results indicate that a first instar can undergo an extended quiescence, but with specific repercussions for doing so. The specific costs of first instar quiescence on the development and starvation tolerance of larvae is ecologically and epidemiologically relevant as mosquito populations in the field often encounter environments with sub-optimal nutritional conditions rendering adult mosquitoes with 
diverse fitness, reproductive potential and vector capacity (Alto et. al., 2005, 2008, Caroci, 2004). Based on the results discussed here, nutrition carried over from maternal reserves into the larval stages is very important for development and likely acts as a buffer against environmental stochasticity or suboptimal conditions.

\subsubsection{The duration of quiescence alters the physiological response to metal stress in newly hatched larvae}

The profound effect of an extended quiescence on newly hatched larvae suggests that other physiological and ecological implications were likely to exist. To explore this possibility newly hatched larvae were exposed to ecological relevant concentrations of copper in order to mimic the metal exposure larvae are likely to experience in urban environments. After 48 hours of an acute exposure, significantly more larvae hatched from old eggs died than those hatched from new eggs. In addition, larvae hatched from old eggs and reared to adulthood in copper exhibited a delayed developmental profile compared to larvae hatched from new eggs along with increased mortality. It is likely that the reductions in nutritional reserves caused by an extended quiescence also affected, at least in part, the response to metal exposure along with the increase in mortality.

The mortality of the larvae reared to adulthood in metal stress experiment was not uniform among the four instar developmental stages. Most mortality occurred during the first instar stage. Larvae that reached the $2^{\text {nd }}$ instar were generally able to reach the pupal stage. This would suggest that first instar mosquitoes are especially sensitive to copper exposure and that rapid tolerance to copper exposure develops in the survivors. A variety of other aquatic and terrestrial insects have been shown to be more sensitive to metals 
during the early instars rather than the late instars further suggesting tolerance develops (Williams et al. 1986; Pascoe et al. 1987; Timmermans et al. 1991).

\subsubsection{The duration of quiescence alters the molecular response to metal stress in newly hatched larvae}

The duration of quiescence alters the response of larval mosquitoes to metal stress at the molecular level. While copper is a critical component of enzymes involved in respiration, oxidative stress protection, pigmentation and iron metabolism, it may also be toxic in excessive concentrations (Egli, et. al., 2006). Our results suggest that in mosquitoes with reduced nutritional reserves copper exposure was much more likely to be fatal. In $A$. aegypti first instars hatched from new eggs $A a \mathrm{Mtn}$ transcription increased rapidly in response to an environmentally relevant concentration of metal in the water. In mosquitoes subjected to an extended quiescence with reduced lipids, the $A a \mathrm{Mtn}$ response to copper exposure was significantly reduced. Detoxification, sequestration and elimination of metals and the neutralization of the oxidative stress associated with metal exposure are nutrient dependent processes (Servia, et al., 2006; Calow, 1991). The exponential increase of Mtn mRNA observed in larvae from new eggs was replaced by a linear response. The compromised nutritional status of larvae from old eggs possibly contributes to this decrease in phenotypic plasticity. While the connection between nutritional reserves, copper exposure and mortality is not fully explained by this study, it is likely that the energetic costs of metal homeostasis in an environment with excessive copper cannot be met in those mosquitoes with reduced nutritional reserves. There was a 
moderate but highly significant increase of $A a \mathrm{Mtn}$ in the absence of metal; an indication of the critical role of this gene in metabolic pathways during early larval development.

Although the reduced metal tolerance and larval development evidenced by these experiments could be the result of a lack of sufficient nutritional reserves, alternative scenarios do exist that can explain reduced growth and higher mortality. Metal ions have been found to chelate with amino acids and proteins and affect their bioavailability in a developing insect; specifically copper interferes with enzymatic processes and peroxidizes polyunsaturated fatty acids (Sivapalan and Guanapragasam, 1980). Other work demonstrated specific reductions in whole body lipids due to metal exposure (Ortel, 1995) as well as fat body and midgut "injury" in some insects (Martoja et al., 1983; Rams-Keller, 1998). While we cannot be certain of the exact mechanism by which copper induced mortality or retarded development in larval mosquitoes, it is likely that one of the contributing factors was the direct effect it had on available nutrients and nutrient storage organs and/or through the additional physiological demands that metal acclimatization induces through the massive up-regulation of the appropriate genes. Either way, the mosquitoes exposed to metal with reduced nutritional reserves due to an extended quiescence had a compromised physiological and molecular response to metal exposure that resulted in higher mortality, delayed development and reduced AaMtn expression.

It has been well described that the regulation of copper import, storage and export is metabolically costly (Servia, et al., 2006). The energetic costs of copper exposure are likely partially manifested through mechanisms capable of chelating, processing and sequestering copper ions. Metallothionein, in particular, has been well established as an 
active participant in the processing of metals (Amiard, et al., 2006; Postuma, and van Straalen, 1993; Roesijadi, 1996). The sequestration of copper involves chelation by Mtn to reduce copper toxicity following a copper shock. Mtn mRNAs were detected as early as 30 min after induction. By four hours there was a very strong up-regulation of Mtn. In our studies, mosquitoes hatched from new eggs and exposed to copper, showed significant mRNA increases in less than an hour, and transcripts exhibited a dramatic 26fold increase over six hrs. Similarly, a moderate 1.5 fold increase in the levels of metallothionein mRNA was described for A. gambiae larvae reared in a copper environment (Mireji, et al., 2010b). Both studies demonstrate a characteristic homeostatic protective response to metal uptake in larval mosquitoes.

\subsubsection{Implications for Vector Ecology and Vector Control}

The presence of mosquitoes has been confirmed in polluted habitats such as drains containing domestic wastewater and in man-made aquatic habitats (Barrera et al., 2008; Chinery, 1995; Mireji, et al 2008,). Aedes aegypti, being a container breeder, may be subject to greater physiological stress from metals in the environment than other species of mosquitoes that transmit disease. This author proposes that newly emerged first instars of aedine mosquitoes hatched from eggs that have been subjected to an extended quiescence such as the dry season are more vulnerable to metal stress during that time. During the wet season, subsequent generations of container breeding mosquitoes that are not experiencing extended egg quiescence, regain their metal tolerance competence. Similar behavior extends to the responses to other forms of stress such as pollution stress, oxidative stress, insecticide metabolism and larval competition 
(Alto, et al., 2005; Poupardin et. al., 2008). As a consequence, extended quiescence could a) contribute to increased vector capacity via increased dengue viral load of adult females derived from quiescent eggs (Alto, et al., 2008) and b) that extended egg quiescence could have implications for vector control strategies, i.e., newly emerged mosquito larvae at the end of the dry season/start of the wet season are physiologically compromised, and therefore potentially more susceptible to vector control strategies than mosquito larvae hatched subsequently throughout the wet season.

\subsection{Acknowledgements}

I would like to thank Dr. Marcela Nouzova, Dr. Jaime Mayoral, Dr. Crisalejandra Rivera and Mark Clifton for critical reading and feedback on the manuscript. This work was supported by NIH/NIGMS R25 GM061347 and the FIU MBRS RISE Biomedical Research Initiative to MHP 


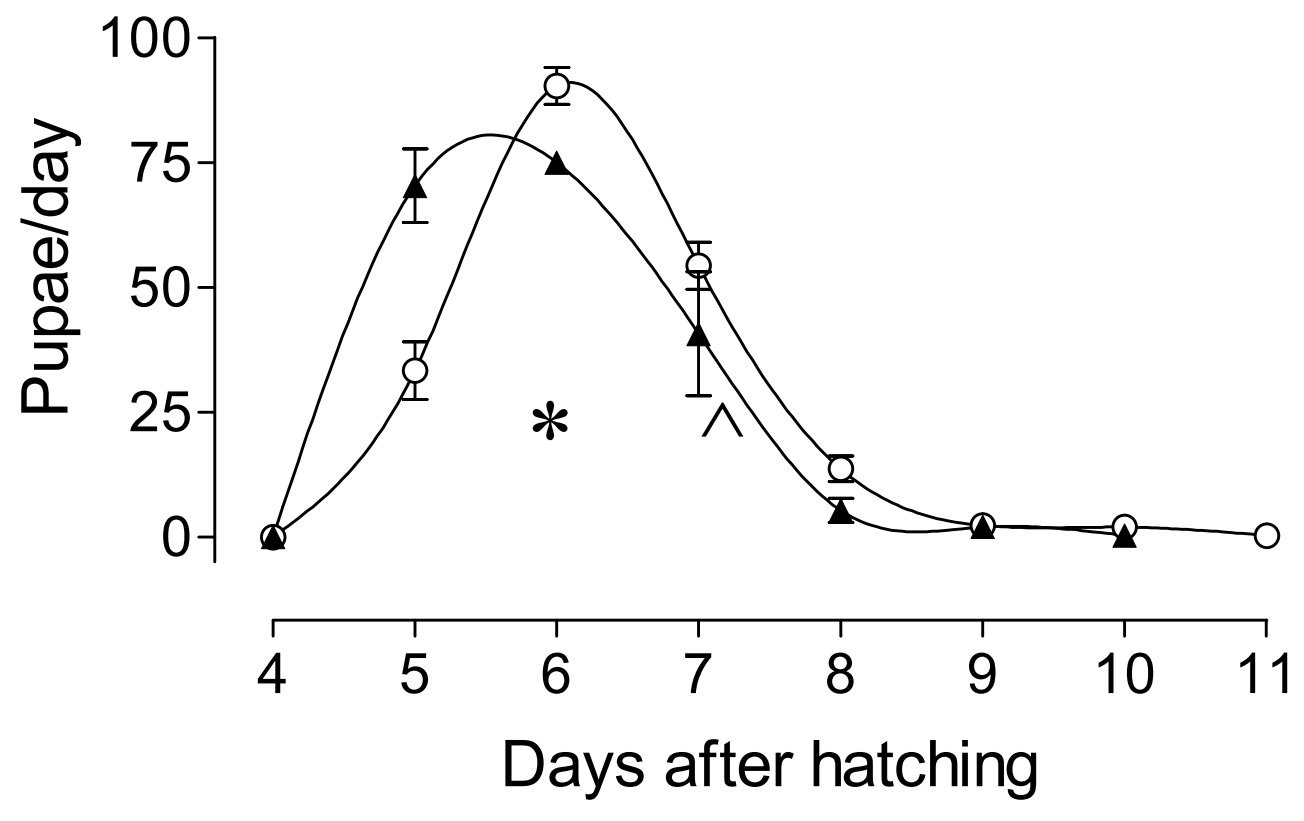

Fig. 11. Larval development period in a clean environment: Larval development period was determined as the mean time to reach pupation for larvae reared in a clean environment. Mean larval development period for larvae from new eggs $=5.95$ days $(*)$ and 7.33 days for larvae from old eggs $\left({ }^{\wedge}\right)$. Each point is the mean \pm SEM of three independent replicates of groups of 200 larvae hatched from new eggs $(\boldsymbol{\Lambda})$ or old eggs $(\bigcirc)$. 


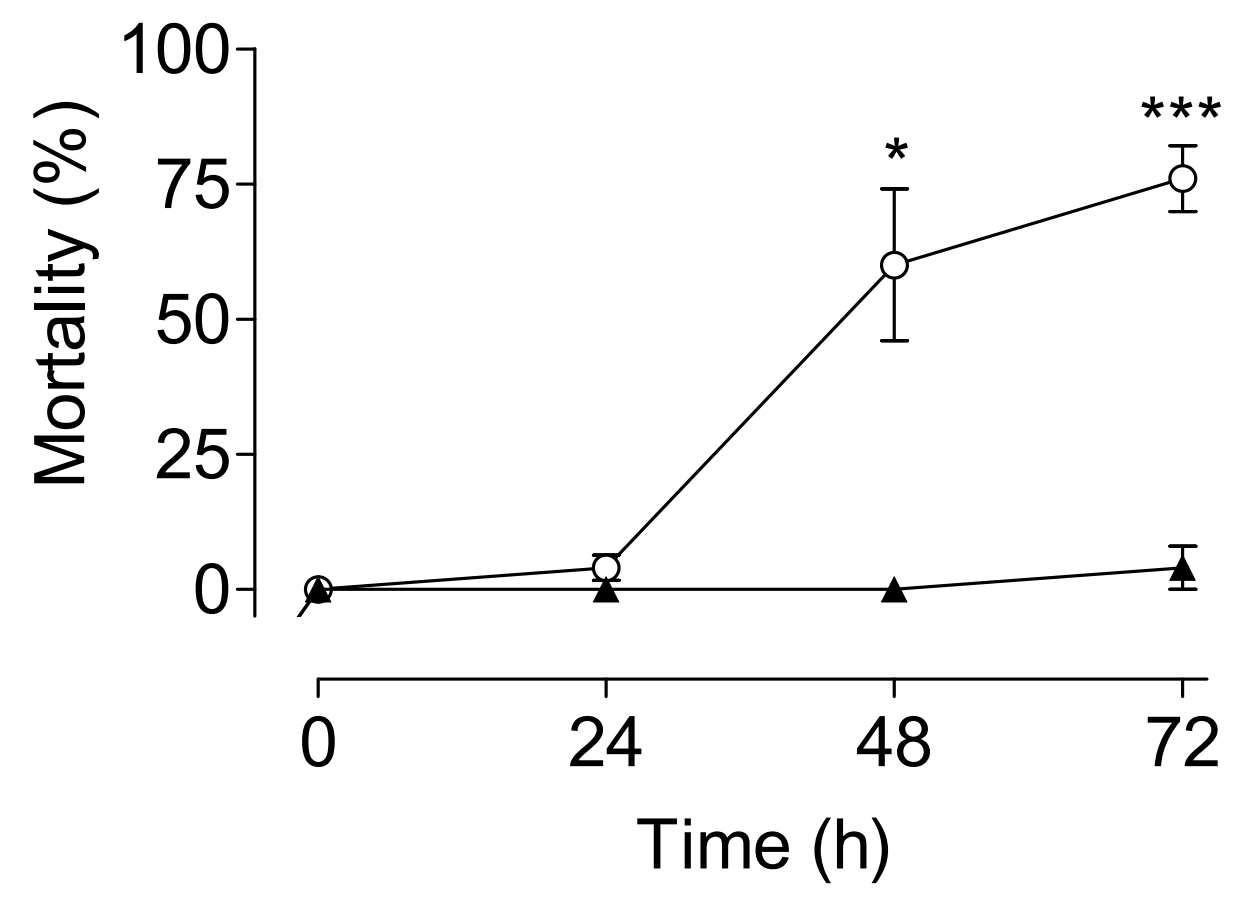

Fig. 12. Larval tolerance to starvation: Mortality of newly hatched larvae in the absence of food was assessed in a 72 hour starvation assay. Each point is the mean \pm SEM of three independent replicates of groups of 25 larvae hatched from new eggs $(\boldsymbol{\Delta})$ or old eggs $(\bigcirc)(* p<0.05$, t test, unpaired, one tailed). 


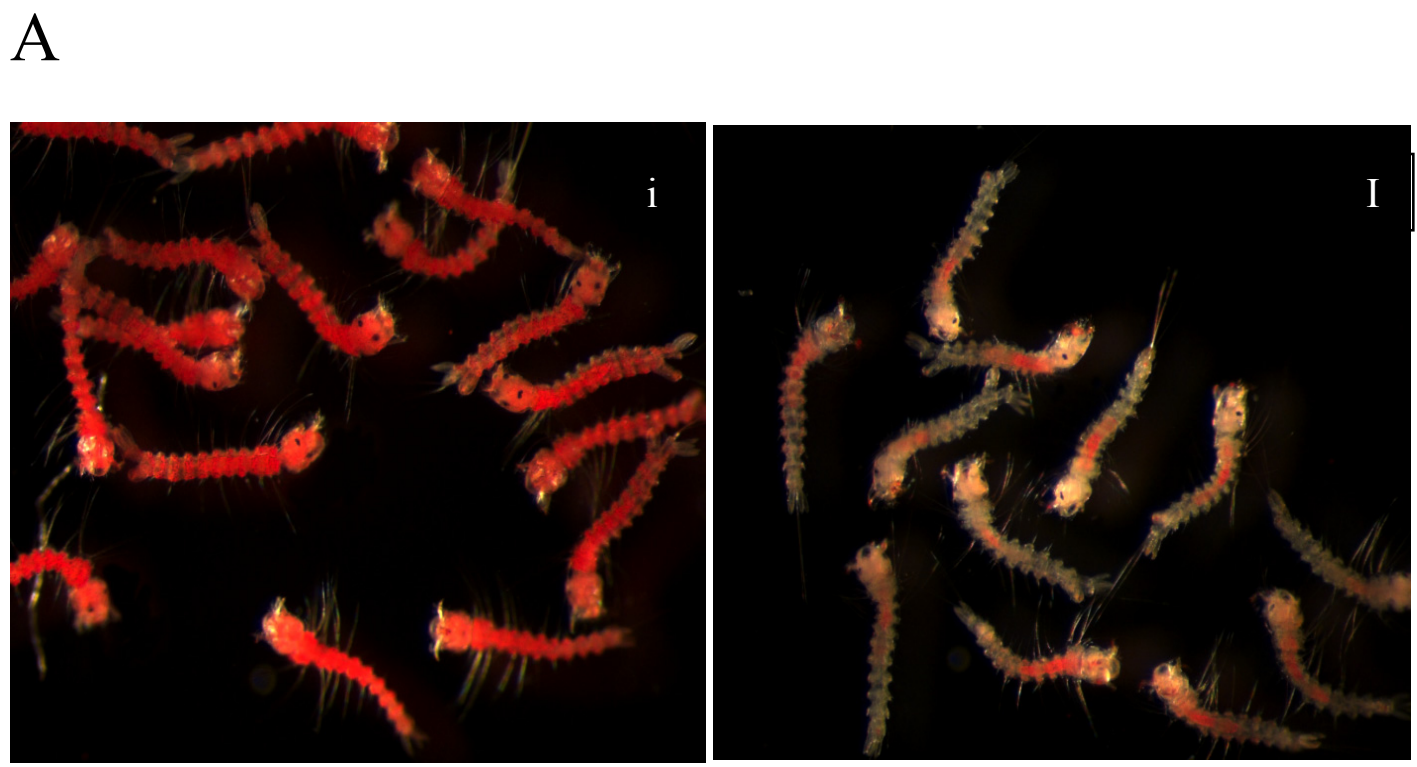

$\mathrm{B}$

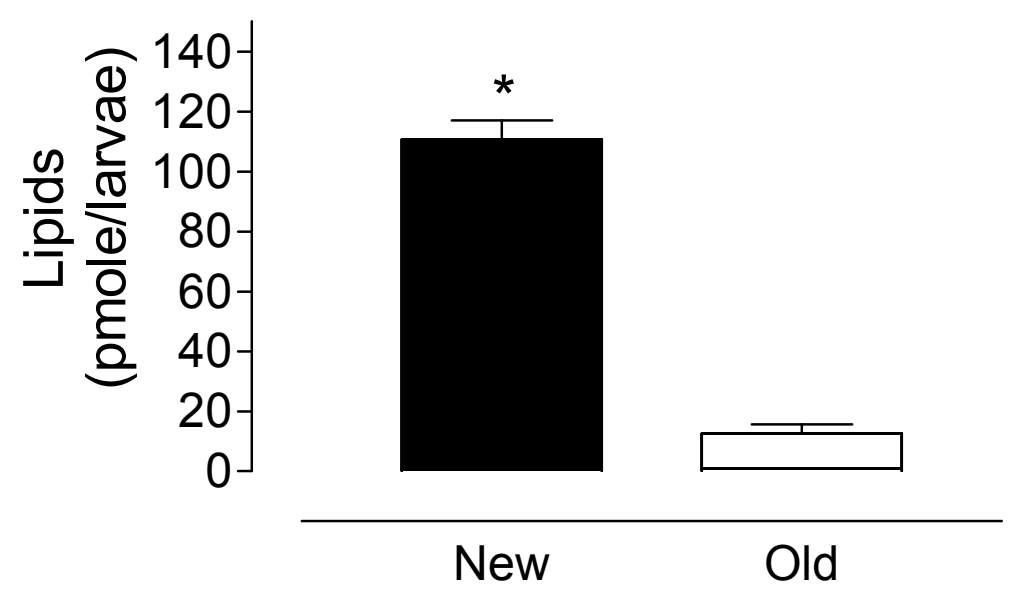

Fig. 13. Larval lipid reserves: A) Oil Red O staining of lipid reserves in newly hatched larvae emerging from new eggs (i) or old eggs (ii). B) Total neutral lipids measured in newly emerged first instars. Results are means \pm SEM of three independent replicates of groups of 90 larvae hatched from new eggs (filled bars) or old eggs (empty bars) (* $\mathrm{p}<0.01$, $\mathrm{t}$ test, paired, one tailed). 


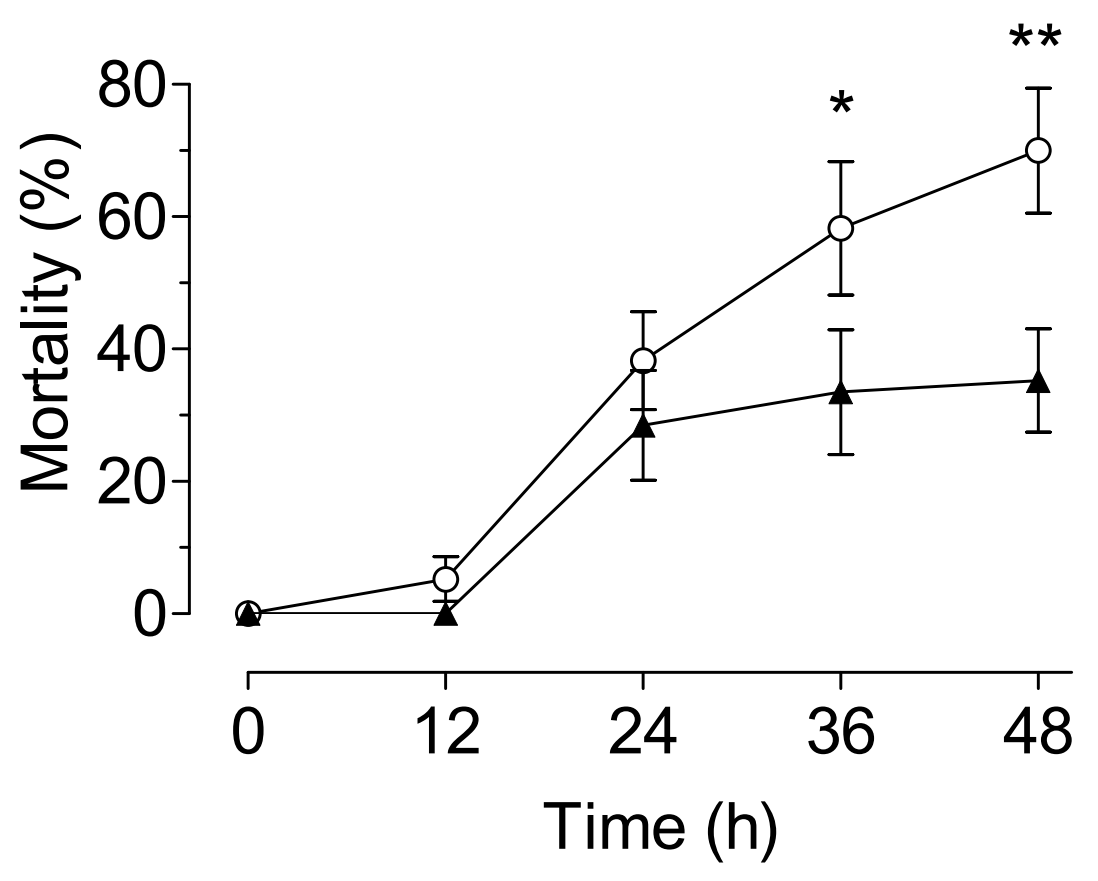

Fig. 14. Larval tolerance to metal stress: Mortality of newly hatched first instar larvae exposed to $1.25 \mathrm{ppm} \mathrm{Cu}$ in a 48 hour acute toxicity assay. Each point is the mean $\pm \mathrm{SEM}$ of three independent replicates of groups of 45 larvae hatched from new eggs $(\boldsymbol{\Delta})$ or old eggs $(\bigcirc)(* \mathrm{p}<0.05$, $\mathrm{t}$ test, unpaired, one tailed $)$. 


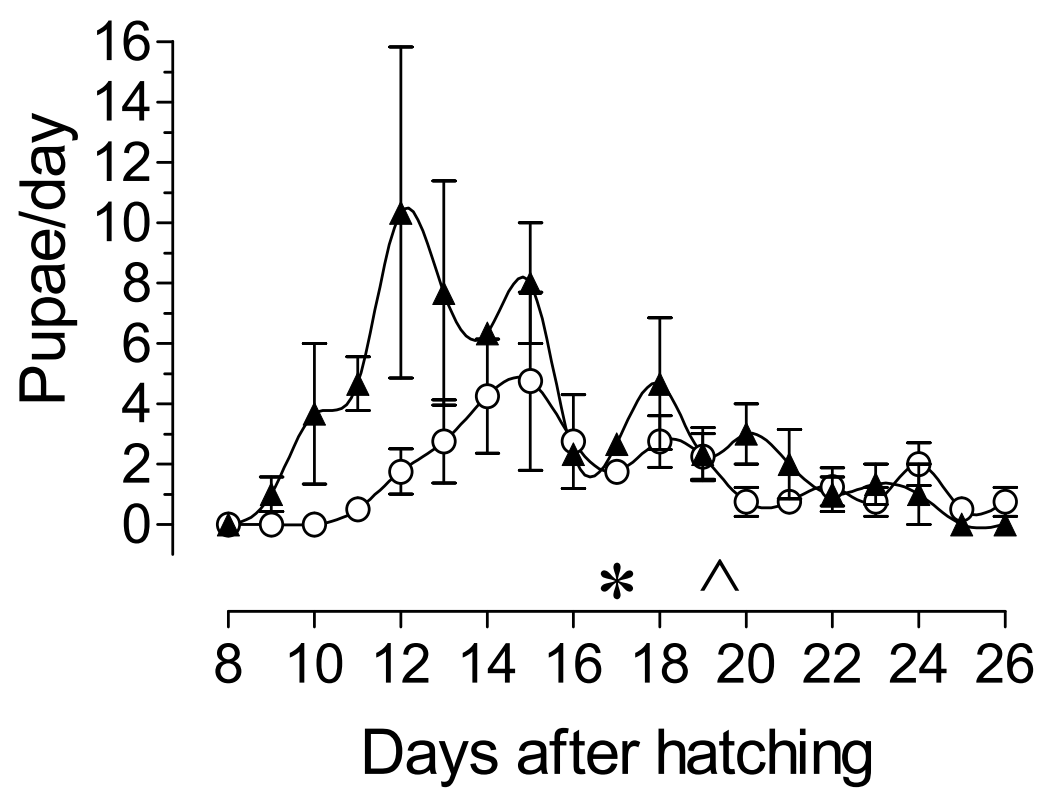

Fig. 15. Larval development period in a metal environment: Larval development period as determined by mean time to reach pupation for larvae reared in $1.00 \mathrm{ppm} \mathrm{Cu}$. Mean larval development period for larvae from new eggs $=15.02$ days $(*)$ and 7.38 days for larvae from old eggs $(\wedge)$. Each point is the mean \pm SEM of three independent replicates of groups of 200 larvae hatched from new eggs $(\boldsymbol{\Delta})$ or old eggs $(\bigcirc)$. 


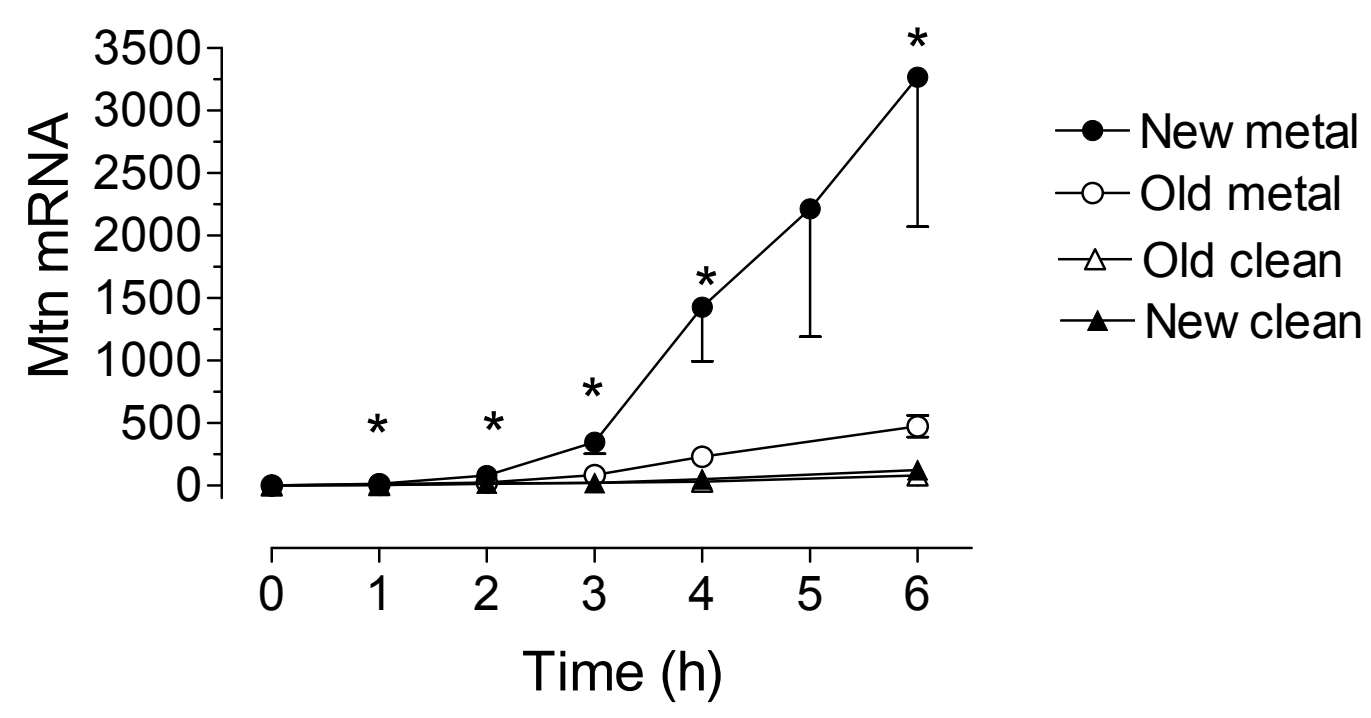

Fig. 16. Metallothionein mRNA levels: Newly hatched larvae were exposed to clean RSW or RSW containing $1.25 \mathrm{pm} \mathrm{Cu}$ for six hours. AaMtn mRNA is expressed as copy number of $A a \mathrm{Mtn}$ mRNA/ 10,000 copies of rpL32 mRNA. Each RT-PCR data point is a mean \pm SEM of three independent biological replicates of 50 larvae $(* \mathrm{p}<0.05, \mathrm{t}$ test, paired, one tailed) (Filled bars are larvae from new eggs; empty bars are larvae from old eggs). 


\subsection{References}

Alekseev, V. R., Ed. (2007). Diapause in aquatic invertebrates : theory and human use Monographiae biologicae Dordrecht ; London : Springer.

Alto, B. W., L. P. Lounibos, et al. (2005). "Larval Competition Differentially Affects Arbovirus Infection in Aedes Mosquitoes." Ecology 86(12): 3279-3288.

Alto, B. W., L. P. Lounibos, et al. (2008). "Larval competition alters susceptibility of adult Aedes mosquitoes to dengue infection." Proceedings of the Royal Society B: Biological Sciences 275(1633): 463-471.

Amiard, J. C., C. Amiard-Triquet, et al. (2006). "Metallothioneins in aquatic invertebrates: Their role in metal detoxification and their use as biomarkers." Aquatic Toxicology 76(2): 160-202.

Andrews, G. K. (2000 ). "Regulation of metallothionein gene expression by oxidative stress and metal ions." Biochem Pharmacol 59: 95-104.

Balamurugan, K., D. Egli, et al. (2007). "Copper homeostasis in Drosophila by complex interplay of import, storage and behavioral avoidance." The EMBO Journal 26(4): $1035-1044$.

Barrera, R., M. Amador, et al. (2008). Unusual productivity of Aedes aegypti in septic tanks and its implications for dengue control. Medical and Veterinary Entomology. 22: 62-69.

Calow, P. (1991). "Physiological costs of combating chemical toxicants: Ecological implications." Comparative Biochemistry and Physiology Part C: Comparative Pharmacology 100(1-2): 3-6.

Caroci, A. S., Y. Li, et al. (2004). Reduced juvenile hormone synthesis in mosquitoes with low teneral reserves reduces ovarian previtellogenic development in Aedes aegypti. Journal of Experimental Biology. 207: 2685-2690.

Chinery, W. A. (1995). "Impact of rapid urbanization on mosquitoes and their disease transmission potential in Accra and Tema, Ghana." Afr. J. Med. Sci 24: 179-188.

Clements, A. N., Ed. (1992). The Biology of Mosquitoes. London, Chapman and Hall.

Crovello, T. J. and C. S. Hacker (1972). "Evolutionary Strategies in Life Table Characteristics Among Feral and Urban Strains of Aedes aegypti (L.)." Evolution 26(2): 185-196. 
Dallinger, R. (1994). "Invertebrate organisms as biological indicators of heavy metal pollution." Applied Biochemistry and Biotechnology 48(1): 27-31.

Egli, D., J. Domenech, et al. (2006). "The four members of the Drosophila metallothionein family exhibit distinct yet overlapping roles in heavy metal homeostasis and detoxification." Genes to Cells 11(6): 647-658.

Hahn, D. A. and D. L. Denlinger (2007). "Meeting the energetic demands of insect diapause: Nutrient storage and utilization." Journal of Insect Physiology 53(8): 760-773.

Hahn, D. A. and D. L. Denlinger (2011). "Energetics of Insect Diapause." Annual Reviews of Entomology 56(1): 103-121.

Huber, K., Y. Ba, et al. (2008). "Aedes aegypti in Senegal: Genetic Diversity and Genetic Structure of Domestic and Sylvatic Populations." Am J Trop Med Hyg 79(2): 218-229.

Kagi, J. H. R. (1991). "Overview of metallothionein." Methods Enzymol 205: 613-626.

Kosalwat, P. and A. W. Knight (1987). "Chronic toxicity of copper to a partial life cycle of the midge,Chironomus decorus." Archives of Environmental Contamination and Toxicology 16(3): 283-290.

Martoja, R., J. M. Bouquegneau, et al. (1983). "Toxicological effects and storage of cadmium and mercury in an insect Locusta migratoria (Orthoptera)." Journal of Invertebrate Pathology 42(1): 17-32.

Mireji, P. O., J. Keating, et al. (2010). "Expression of metallothionein and [alpha]-tubulin in heavy metal-tolerant Anopheles gambiae sensu stricto (Diptera: Culicidae)." Ecotoxicology and Environmental Safety 73(1): 46-50.

Mireji, P. O., J. Keating, et al. (2010). "Biological cost of tolerance to heavy metals in the mosquito Anopheles gambiae." Medical and Veterinary Entomology 24(1).

Mireji, P. O., J. Keating, et al. (2008). "Heavy metals in mosquito larval habitats in urban Kisumu and Malindi, Kenya, and their impact." Ecotoxicology and Environmental Safety 70(1): 147-153.

Munstermann, L. E. and L. M. Wasmuth, Eds. (1985). Handbook of Insect Rearing. Amsterdam, Elsevier

Nishiura, J. T., C. Burgos, et al. (2007). "Modulation of larval nutrition affects midgut neutral lipid storage and temporal pattern of transcription factor expression during mosquito metamorphosis." Journal of Insect Physiology 53(1): 47-58. 
Noriega, F. G. and M. A. Wells (1993). "A comparison of three methods for isolating RNA from mosquitoes." Insect Molecular Biology 2(1): 21-24.

Ortel, J. (1995). "Effects of metals on the total lipid content in the gypsy moth (Lymantria dispar, lymantriidae, lepid.) and its hemolymph." Bulletin of Environmental Contamination and Toxicology 55(2): 216-221.

Pascoe, D., K. A. Williams, et al. (1989). "Chronic toxicity of cadmium to Chironomus riparius Meigen - effects upon larval development and adult emergence" Hydrobiologia 175(2): 109-115.

Posthuma, L. and N. M. Van Straalen (1993). "Heavy-metal adaptation in terrestrial invertebrates : a review of occurrence, genetics, physiology and ecological consequences." Comparative biochemistry and physiology. C. Comparative pharmacology and toxicology 106(1): 11-38

Poupardin, R., S. Reynaud, et al. (2008). "Cross-induction of detoxification genes by environmental xenobiotics and insecticides in the mosquito Aedes aegypti: Impact on larval tolerance to chemical insecticides." Insect Biochemistry and Molecular Biology 38(5): 540-551.

Rayms-Keller, A., K. E. Olson, et al. (1998). "Effect of Heavy Metals on Aedes aegypti (Diptera:Culicidae) Larvae." Ecotoxicology and Environmental Safety 39(1): 4147.

Roesijadi, G. (1996). "Metallothionein and its role in toxic metal regulation." Comparative Biochemistry and Physiology Part C: Pharmacology, Toxicology and Endocrinology 113(2): 117-123.

Sarkar, S., A. K. Duttagupta, et al. (2004). "Effects of heavy metals on population growth and metallothionein gene expression in the mosquito Culex quinquefasciatus, from Calcutta, India." Environmental Pollution 127(2): 183-193.

Servia, M., A. Péry, et al. (2006). "Effects of copper on energy metabolism and larval development in the midge Chironomus riparius " Ecotoxicology 15(3): 229-240.

Sivapalan, P. and N. C. Gnanapragasam (1980). "Influence of copper on the development and adult emergence of Homona coffearia; (Lepidoptera: Tortricidae) reared in vitro." Entomologia Experimentalis et Applicata 28(1): 59-63.

Sorvari, J., L. M. Rantala, et al. (2007). "Heavy metal pollution disturbs immune response in wild ant populations." Environmental Pollution 145(1): 324-328. 
Timmermans, K. R., W. Peeters, et al. (1992). "Cadmium, zinc, lead and copper in Chironomus riparius (Meigen) larvae (Diptera, Chironomidae): uptake and effects." Hydrobiologia 241(2): 119-134.

Trpis, M. and W. Hausermann (1975). Demonstration of differential domesticity of Aedes aegypti (L.) (Diptera, Culicidae) in Africa by mark-release-recapture, Bulletin of Entomological Research. 65: 199-208.

Van Handel, E. (1993). "Fuel metabolism of the mosquito (Culex quinquefasciatus) embryo." Journal of Insect Physiology 39(10): 831-833.

Weissman-Strum, A. and S. H. Kindler (1962). "Effect of Low Temperatures on Development, Hatching and Survival of the Eggs of Aedes aegypti (L)." Nature 196(4860): 1231-1232.

WHO (2005). Communicable Disease Control: Guidelines for Laboratory and Field Testing of Mosquito Larvicides.

Williams, K. A., D. W. J. Green, et al. (1986). "Cadmium, zinc, lead and copper in Chironomus riparius (Meigen) larvae (Diptera, Chironomidae): uptake and effects." Oecologia 70(3): 362-366. 


\section{Chapter 5: Aedes aegypti pharate first instar quiescence: A case for anticipatory reproductive plasticity}

\subsection{Abstract}

Aedes aegypti mosquitoes use pharate first instar quiescence to cope with fluctuations in water availability hosting a fully developed first instar larvae within the chorion. The duration of this quiescence has been shown to affect larval fitness. This study sought to determine if extended egg quiescence can elicit a plastic response resulting in an adult phenotype distinct from adults reared from short quiescence eggs. Our findings indicate that extended pharate first instar quiescence affects the performance and reproductive fitness of the adult female mosquito as well as the nutritional status of its progeny via maternal effects in an adaptive manner. This study demonstrates that phenotypic plasticity results as a consequence of the duration of pharate first instar quiescence and alternative phenotypes may exist for this mosquito with quiescence serving as a cue possibly signaling the environmental conditions that follow a dry period. These findings have implications for $A$. aegypti's success as a vector, geographic distribution, vector capacity and control.

\subsection{Introduction}

Phenotypic plasticity is the ability of a single genotype to produce more than one alternative form of morphology, physiological state, and/or behavior in response to environmental conditions; it can subsequently result in alternative phenotypes - two or more forms of behavior, physiological response, or structure maintained in the same life 
stage in a single population and not simultaneously expressed in the same individual (West-Eberhard, 2003). In the case of anticipatory plasticity, phenotypic changes are induced in response to cues which signal future environmental conditions prior to the actual onset of these conditions (Esperk et al., 2012). In tropical and subtropical regions mosquitoes are often exposed to a favorable rainy season with abundant breeding sites and vegetation, and a stressful drier winter (Vinogradova, 2007; Denlinger, 1986; Mori et al., 1981) during which resources may be less plentiful (Gary and Foster, 2001; Foster, 1995; Yaro, et al., 2012). Regardless of the season fluctuations in moisture are however, unpredictable. Aedes aegypti mosquitoes have the ability to undergo aseasonal dormancy as an unhatched first instar; this dormant state is referred to as pharate first instar quiescence or more simply, egg quiescence (Vinogradova, 2007; Clements, 1992). Aseasonal quiescence is an adaptation to the peculiar conditions of special larval habitats, such as tree and rock holes, or transient ground pools, where water level may be subjected to large and abrupt fluctuations (reviewed in Vinogradova, 2007). As a result of this trait, A. aegypti produce eggs that resist desiccation and can withstand months of dormancy.

A wide variety of studies have shown that the energetic costs of long-term diapause are reflected in lower post-hatching survival and reduced fecundity (reviewed in Hahn and Denlinger, 2007, 2011), however, relatively little work has been conducted examining the fitness costs and consequences of egg quiescence. The duration of extended pharate first instar quiescence has been shown to affect larval fitness by reducing nutritional reserves of newly emerged first instars, increasing developmental period and decreasing their stress tolerance (Perez and Noriega, 2012). These 
observations led us to hypothesize that the duration of egg quiescence would result in adaptive adult phenotypes with distinctly different performance and reproductive physiology. To test this hypothesis a laboratory strain of $A$. aegypti adults reared from extended quiescence eggs were assessed for various parameters of performance and reproductive physiology and compared with adults reared from short quiescence eggs. Our findings indicate that extended egg quiescence produced adult females with a distinct phenotype from those that had undergone short egg quiescence i.e., altered performance and reproductive fitness as well as altered nutritional status of their progeny via maternal effects, perhaps in a manner best suited for the likely suboptimal conditions that follow extended quiescence. These results suggest that alternative phenotypes may exist for this mosquito with implications for its success as a vector, geographic distribution, vector capacity and control.

\subsection{Materials and Methods}

\subsubsection{Mosquito rearing and maintenance}

A. aegypti (Rockefeller strain) short quiescence pharate first instars (eggs less than a week old) were hatched in deoxygenated water. Upon emergence, three replicate groups of 200 of these larvae were reared to adulthood in $23 \times 40 \times 15 \mathrm{~cm}$ polypropylene plastic pans containing 1L of reconstituted soft water (RSW) (Perez and Noriega, 2012). For the metal stressed treatment, three replicate groups of 200 first instar larvae from short quiescence eggs were reared to adulthood in one ppm copper $\left(\mathrm{Cu}^{2+}\right)$ in RSW. One ppm $\mathrm{Cu}^{2+}$ has been shown to be an ecologically relevant level of metal stress (Sarkar et al., 2004). Larval diet consisted of $750 \mu 1$ of $10 \%$ liver powder suspension (MP 
Biomedicals Inc., Aurora, OH.) added to the pan every day except for the second day. Pupae were collected every day throughout the pupation period and transferred to an appropriate container for adult emergence. Adult mosquitoes were offered a cotton pad soaked in $3 \%$ or $20 \%$ sucrose solution ad libitum. These concentrations represented relative sub-optimal and optimal adult environments for comparison purposes. Four-dayold female mosquitoes were fed porcine blood equilibrated to $37^{\circ} \mathrm{C}$, and ATP was added to the blood meal to a final concentration of $1 \mathrm{mM}$ immediately before use. Eggs were oviposited on papers and stored under insectary conditions in Nasco Whirl-Pak bags inside a resealable polypropylene container (adapted from Munstermann, 1985). Insectary conditions were $28{ }^{\circ} \mathrm{C}$ and $80 \%$ relative humidity under a photoperiod of $16 \mathrm{~h}$ light and $8 \mathrm{~h}$ dark. Extended quiescence eggs were generated by storing the remaining eggs of the same cohort under insectary conditions for ten weeks. All experiments were then repeated with these 'old' eggs. All assays were performed under insectary conditions and started at $\sim 12: 00 \mathrm{pm}$.

\subsubsection{Reagents}

Reconstituted soft water (RSW) was used in all bioassays. RSW was prepared by adding reagent-grade $\mathrm{NaHCO}_{3}(48 \mathrm{mg}), \mathrm{CaSO}_{4} 2 \mathrm{H}_{2} \mathrm{O}(30 \mathrm{mg}), \mathrm{MgSO}_{4}(30 \mathrm{mg})$ and $\mathrm{KCl}$ $(2 \mathrm{mg})$ to 1 liter of deionized water ( $\mathrm{pH} 7.4)$. All reagents were purchased from Fisher Scientific, Pittsburgh, PA. 


\subsubsection{Copper measurement}

Reagent grade copper sulfate pentahydrate was dissolved in RSW. Nominal $\mathrm{Cu}^{2+}$ concentrations were confirmed by inductively coupled plasma mass spectrometric analysis (ICP-MS) using an HP 4500 plus IPC-MS instrument (Hewlett-Packard Co., Wilmington, DE), equipped with a Babington-type nebulizer and an ASX-500 autosampler (Cetac Technologies Inc., Omaha, NE).

\subsubsection{Fitness assays}

Newly emerged adult females from each treatment were collected, dried at $95^{\circ} \mathrm{C}$ for $24 \mathrm{~h}$ and analyzed gravimetrically with a Cahn 29 electrobalance to within one 1/100 of a milligram. For the starvation tolerance assay three replicate groups of 15 newly emerged adult females from each treatment were assayed in one pint mosquito cages and provided deionized water ad libitum. The longevity assay was performed in a similar manner except $3 \%$ sucrose was provided. For the filial larvae starvation tolerance assay (maternal effects) three replicate groups of 25 newly emerged first instars generated from parents that had been reared from short quiescence and extended quiescence eggs were assayed in $15 \mathrm{~cm}$ diameter glass petri dishes containing $100 \mathrm{ml}$ of RSW in the absence of food. The number of larvae found not responsive to mechanical stimulation every $24 \mathrm{~h}$ in each assay were counted as "dead" and removed. 


\subsubsection{Quantification of total neutral lipids}

Neutral lipids were quantified using a triglyceride quantification kit (Biovision, Mountain View, CA; cat\# K622-100). Three newly emerged adult females from each treatment were assayed in triplicate.

\subsubsection{Quantification of follicles per ovary}

A sub-sample of adults was analyzed for follicle quantification. $60-72 \mathrm{~h}$ old adult females were anesthetized by chilling for ten min at $4^{\circ} \mathrm{C}$ prior to dissection. Ovaries from these mosquitoes were removed, rinsed in Aedes physiological saline (APS) $\left(\mathrm{MgCl}_{2} 0.6\right.$ mM; KCl 4.0 mM NaHCO 1.8 mM; NaCl 150.0 mM; HEPES 25.0 mM; $\mathrm{CaCl}_{2} 1.7 \mathrm{mM}$ ) and stained with $0.5 \%$ neutral red solution in acetate buffer at pH 5.2 (Sigma-Aldrich, St. Louis, MO) for $5 \mathrm{~s}$ to more easily visualize follicles (Clifton and Noriega, 2011). The ovaries were rinsed a second time in APS and placed under a coverslip. Photographs were taken of the previtellogenic ovaries using a DM 5500 B Leica fluorescence microscope, a Leica DFC 310 FX mounted camera and Leica LAS imaging software. Ovaries were later scored using Leica LAS imaging software for total follicle count. A mean follicle value was then calculated to represent previtellogenic follicle output for the cohort.

\subsubsection{Quantification of eggs}

Mated mosquitoes were offered a blood meal as described above (sec. 2.1). Although blood meal volume was not directly quantified, after feeding each female was 
visually inspected and judged to have fed to satiety by her fully engorged abdomen. Fifteen fully engorged females were housed in individual containers (one female/container) and provided 3\% sucrose ad libitum. Ninety-six h after blood feeding egg papers were collected, dried under insectary conditions, allowed five days to complete embryogenesis and photographed with a digital camera. The number of eggs produced per female was later determined by manually counting the number of eggs on each egg paper from those photographs. A mean was then calculated to represent egg output for the cohort.

\subsubsection{Estimation of follicle resorption}

The extent to which follicles were resorbed (expressed as a percentage) was determined by subtracting the mean number of oviposited eggs/female from the mean number follicles/female from each treatment combination.

\subsubsection{Quantification of fertility}

Fertility (number of viable offspring) was used as an estimate of reproductive fitness. Eggs collected in section 2.7 were hatched as previously described and the number of newly emerged first instars counted.

\subsubsection{Data analysis}

Graphical representations and statistical analysis were performed with GraphPad Prism Software (San Diego, CA) version 6.00 for Windows. Results were expressed as means and SEM. Survivorship $\left(\mathrm{LT}_{50}\right)$ was determined by non-linear regression curve fit. 
Significant differences in survivorship $\left(\mathrm{LT}_{50}\right)$ were determined with a log rank method. Significant differences for all other results were determined with a one tailed student's $t$ test or in the case of reproductive output, for graphical clarity, a one way ANOVA with a Tukey's test for multiple comparisons was used $(P<0.05)$.

\subsection{Results}

\subsubsection{Extended quiescence altered female adult performance}

Extended quiescence reduced female adult body mass - Adult female dry body mass at time of emergence was significantly reduced by $21 \%$ when compared with their short quiescence counterparts indicating that an egg quiescence period of ten weeks results in a morphometrically altered adult female. A significant difference in the dry body mass of the larval metal stress treatments suggested a difference in metal physiology response occurred between extended and short quiescence females (Fig. 17).

Extended quiescence increased starvation tolerance of female adults - Tolerance to starvation, as estimated by mean survival time $\left(\mathrm{LT}_{50}\right)$, was $12 \%$ greater in newly emerged adult females reared from extended quiescence eggs than adult females reared from short quiescence eggs. Larval metal stress reduced starvation tolerance significantly more in adult females from short quiescence eggs (20\% vs. $11.2 \%)$ indicating a difference in metal tolerance physiology between the two phenotypes with females from extended quiescence being more tolerant of metal stress (Fig. 18A). Taken together these results indicate that females reared from extended quiescence eggs are more tolerant of short term or immediate stress. Mean neutral storage lipid reserves (NSL, nmoles/female) between extended quiescence and short quiescence treatments also differed significantly 
in correlation with the starvation tolerance results, i.e. the more NSL, the higher the $\mathrm{LT}_{50}$ value (Fig. 18B and inset).

Extended quiescence increased longevity of adult females - The life span or mean survival time $\left(\mathrm{LT}_{50}\right)$ of unmated adult females was $\sim 10 \%$ greater for females reared from extended quiescence eggs than for females reared from short quiescence eggs suggesting

altered physiological states between the two. Larval metal stress had a hormetic effect in both extended and short quiescence treatments further increasing lifespan in each case, however, the magnitude of the effect was significantly greater in females from short quiescence (20 vs. 12\%) (Fig. 19).

\subsubsection{Extended quiescence altered reproductive strategy}

Effect of extended quiescence on reproduction: Adult females reared from extended quiescence eggs developed significantly less resting stage follicles than females reared from short quiescence eggs, yet laid more eggs and yielded $\sim 14 \%$ more viable offspring under the sub optimal 3\% sucrose regime (Fig. 20A). Altered follicular resorption is responsible for this difference; under the sub optimal $3 \%$ sucrose regime females reared from extended quiescence eggs resorbed $22 \%$ of the resting stage follicles while females reared from short quiescence eggs resorbed 39\%. However, when adult females reared from short quiescence eggs were given the enriched $20 \%$ sucrose diet they resorbed only $23 \%$ of their follicles and the subsequent number of eggs developed was similar to those of females reared from quiescent eggs (Fig. 20B). 


\subsubsection{Response to larval metal stress}

Larval metal stress reduced the number of resting stage follicles similarly in females reared from both extended and short quiescence eggs (14.5\% vs. 11.7\%), however, the mean number of eggs matured and viable offspring were dramatically reduced ( $>5 \mathrm{x}$ fold) only in the extended quiescence phenotype (Fig. 20A). Reproduction in extended quiescence females was particularly sensitive to larval metal stress whereas the opposite held true for the other forms of stress analyzed.

\subsubsection{Extended quiescence produced maternal effects}

Extended quiescence reduced tolerance to starvation of offspring. The maternal effects of extended quiescence were observed in the reduced starvation tolerance of newly emerged first instar progeny of adults reared from eggs having under gone quiescence $(11.9 \%)$. The effect of larval metal stress on maternal effects for these two treatments differed only slightly, although significantly (20.0 vs. $16.0 \%)$ suggesting that P1 generation may also be phenotypically different (Fig. 21).

\subsection{Discussion}

Rainfall pattern is the most conspicuous aspect of tropical seasons. The wet/dry cycle is responsible for the seasonal growth of vegetation and small puddles that provide suitable habitats for container breeding mosquitoes. The subsequent increase in plant growth provides carbohydrate rich food resources for many phytophagous insects that during the drier season are less abundant. Egg quiescence occurs in mosquitoes of the genera Ochlerotatus, Aedes, and Psorophora; these tropical and sub-tropical container 
inhabiting species face the challenge of unpredictable alternating dry and wet periods utilizing egg quiescence to survive dry conditions (Vinogradova, 2007). Our results suggest that $A$. aegypti egg quiescence may serve as a cue signaling the sub-optimal adult environmental conditions that follow a dry period. The resulting adult phenotype is subsequently better fit for these conditions. This unique characteristic may have contributed to its wide geographic distribution and success as a vector.

\subsubsection{Quiescence and female adult performance}

Our observation that $A$. aegypti adult female mosquitoes reared from extended quiescence eggs have smaller body mass at emergence, yet more lipid reserves and greater fecundity than their short quiescence counterparts might seem to contradict the current literature stating that female body size, lipid reserves and fecundity are all positively correlated (Caroci, et al., 2004; Blackmore and Lord, 2000; Briegel, 1990). However insects, being capable of a high degree of phenotypic plasticity, including seasonal polyphenism, are able to adjust the relative proportion of teneral reserves so as to develop phenotypes best suited to environmental changes (Brakefield, et al., 2007, Moran, 1992; West-Eberhardt, 2003). It has also been demonstrated that altered insulin signaling in Drosophila melanogaster can produce a phenotype with smaller somatic body size, hypertrophied fat bodies with high lipid stores that are long lived (Tatar et al.,

2001). Altered metabolic rate may also be responsible. It has been shown in a Lepidopteron model that insect body size is proportional with metabolic rate (Brakefield, et al., 2007; Pijge, et al., 2007) and a high metabolic rate would entail greater maintenance requirements resulting in lower adult quality (Gotthard et al., 1994). 
These results also demonstrated that unmated adult female mosquitoes reared from extended quiescence eggs exhibited greater starvation tolerance and longer lifespan than females reared from short quiescence eggs. Many organisms encounter periods of starvation in their life and most organisms have evolved some form of adaptive physiology to cope with them. It has often been suggested that the mechanisms that enhance survival under starvation also underlie the regulation of longevity. In a range of well-studied animal species, long-lived individuals appear to be more resistant to multiple stresses, often including starvation (Pijpe et al., 2007). Mosquitoes emerging at a dry/wet period interface with greater adult lipid reserves, starvation tolerance and longevity would be at a physiological advantage at a time when environmental conditions may be more challenging than during the wet season. Previous work has already demonstrated that larvae hatched from extended quiescence eggs exhibit a longer larval developmental period and presumably a slower growth rate than larvae hatched from eggs having undergone a brief quiescence (Perez and Noriega, 2012). Therefore, it may be possible that smaller mosquitoes with a lower metabolic rate can emerge with greater neutral lipid reserves, altered metabolism and increased adult survivorship. These individuals emerging at the dry/wet season interface might have been physiologically reprogrammed by the extended quiescence period for improved performance and fitness during the weeks that follow. Alternatively, extended quiescence may increase adult longevity by acting as a period of larval dietary restriction. Periods of famine often trigger what appear to be metabolic switches that paradoxically extend the normal life span, without sacrificing subsequent reproduction and survival when favorable conditions return (Austad, 1989; Kirkwood and Austad, 2000). For example, dietary restriction in $A$. 
aegypti larvae has been shown to extend adult lifespan (Joy, et al., 2010). Dietary restriction is thought to increase lifespan though lowered metabolic rate and decreased TOR signaling as an active, highly conserved stress response that evolved early in life's history to increase an organism's chance of surviving adversity (Britton, et al., 2002; Powers, et al., 2006; Sinclair, 2005).

A. aegypti larval breeding sites are heterogeneous and dynamic environments with metals and other physiological stressors (Barrera et al., 2006, 2008; Huber et al., 2008). Larval environmental conditions can alter the growth and development of mosquitoes and subsequently their adult phenotypes, reproduction and progeny (Blackmore and Lord, 2000; Caroci, et al., 2004; reviewed in Merrit et al., 1992). Therefore, the response to larval metal stress on these two adult phenotypes was examined. Larval environmental metal stress resulted in reduced body mass and lipid reserves that were most likely responsible for reduced starvation tolerance in adults reared from both extended quiescence and short quiescence eggs, however the response was greater in the short quiescence condition. This overall reduction in performance in both phenotypes is most likely a result of the caloric cost of metal detoxification, sequestration and elimination (Mireji et al., 2010; Pook, et al., 2009; Servia, et al., 2006; Perez and Noriega, unpublished), but why the response was greater in short quiescence adults is unclear. A hormetic effect was also observed which was greater in adults reared from short quiescence eggs the those from extended quiescence eggs. Hormesis is defined as a favorable biological response to low exposures of a stressor (reviewed in Sinclair, 2005). Longevity has been shown to be increased by moderate metal or oxidative stress in 
several studies with D. melanogaster and C. elegans (Baldal et al., 2006; Barsyte et al., 2001; Broughton et al., 2005).

\subsubsection{Extended quiescence alters reproductive success}

We have previously shown that an extended quiescence can be metabolically expensive for $A$. aegypti larvae and in that regard quiescence is similar to diapause. Unlike diapause which results in lower post diapause fecundity, our results suggest that the extended quiescence period of first instars adjusts the reproductive strategy of adults in a manner that might help reestablish local mosquito populations at the end of a dry period, an unfavorable time which requires maximum fertility, i.e., maximum viable offspring. This altered reproductive performance, however, comes at a cost, i.e., maternal effects; first instar larvae from females reared from quiescent eggs are less fit.

Reproductive trade-offs in adult mosquitoes occur primarily through the process of oosorption (Clifton and Noriega, 2011 and 2012). By resorbing excess reproductive tissues, insects can alter previous reproductive decisions by redirecting resources away from reproduction in favor of competing physiological activities. Y-model allocation theory predicts that a limited resource is divided between survival and reproduction (e.g. starvation resistance and longevity vs. fecundity). The costs of reproduction involving trade-offs between egg production and mortality are well documented in insects and other animals with trade-offs imposing constraints among survival, growth, resource acquisition and reproduction; nutrient limitation being the central mechanism underlying these trade-offs (Boggs, 2009; Chippindale, et al., 1993). Seasonal variation in the 
reproductive parameters of Anopheles gambiae have also been demonstrated (Yaro, et al., 2012).

Adult $A$. aegypti females derived from extended quiescence and short quiescence eggs and fed the sub-optimal 3\% diet resorbed dramatically different proportions of their follicles $(22 \%$ vs. $39 \%$ respectively). However, when females reared from short quiescence eggs where fed the enriched $20 \%$ diet they resorbed only $23 \%$ of their follicles. From these data we conclude that adult females reared from short quiescence eggs and fed the sub-optimal 3\% sugar diet failed to predict their adult environment and had to resorb excess reproductive material. In a natural setting, females derived from short quiescence eggs would have expected an optimal environment that the wet season would provide or that the $20 \%$ sucrose diet represents.

The reduction in the number of resting stage follicles developed under both quiescence conditions produced by larval metal stress is most likely due to a reallocation of resources due to the above mentioned 'cost' of metal stress. The number of ovarioles a female will produce is determined during the $2^{\text {nd }}-3^{\text {rd }}$ instar stage (Ronquillo and Horsefall, 1969). It is reasonable to hypothesize that newly emerged larvae sensing a stressful environment will allocate resources to somatic rather than reproductive purposes (reviewed in Boggs, 2009; Postma et al., 1995; Servia, 2006). Most interestingly, larval metal stress reduced fecundity and fertility dramatically only after extended quiescence. Although adult females reared from extended quiescence eggs are apparently better fit than females reared from short quiescence eggs in the absence of a stressor; in the presence of a stressor the opposite holds true. The reasons for this are unclear; however, 
this is strong evidence that adult females reared from extended quiescent eggs are physiologically distinct from females reared from short quiescence eggs.

\subsubsection{Extended quiescence and maternal effects}

Extended quiescence reduced tolerance to starvation of offspring first instar larvae under both clean and metal stressed conditions. The fertilized mosquito egg is a closed system totally dependent on maternally-derived lipid reserves to complete embryogenesis and maintain its quiescent metabolism (Clements, 1992; Vinogradova, 2007). As a consequence, a nutrient limited adult has fewer resources to devote to reproduction and therefore less maternally derived nutrients are passed on to filial larvae. Maternal effects, therefore, reflect the nutritional status of the parents, i.e., filial pharate first instar larvae derived from extended quiescence and containing less nutrient reserves will perform accordingly. Adult females reared from extended quiescence eggs exhibited greater fertility than adult females reared from short quiescence eggs at the cost of the next generation when presumably environmental conditions might improve. Then, if mesic conditions follow, females will produce eggs with higher lipid content enabling those eggs to endure a prolonged quiescence to survive any subsequent dry period.

\subsubsection{Implications for Vector Ecology}

Aseasonal pharate first instar quiescence is an adaptation to the large and abrupt fluctuations in water availability of larval container habitats. It results in an asynchronous hatching of eggs. Usually the first flooding induces hatching of the majority of the eggs, whereas the remainder of the eggs may hatch much later after a subsequent flooding 
episode. Asynchronous hatching is based on intrapopulation variation in sensitivity of individuals to environmental cues. Such arrest of hatching is a very useful adaptation favoring conservation of populations with unpredictable water sources (Vinogradova, 2007 and references therein,). Such an adaptation may have inadvertently contributed to A. aegypti's wide geographic distribution and success as an anthropophilic vector. Furthermore, egg quiescence that results in adult plasticity would give this species of mosquito a great advantage in colonizing and maintaining population abundances in new geographic areas by producing adults best suited to novel environments. Although $A$. aegypti is considered a tropical mosquito and its distribution appears to be influenced by climate in some temperate regions of the world, contemporary and historical records document persistence outside these regions (Christophers, 1960). The peridomestic behavior of $A$. aegypti and its desiccation-resistant eggs afford this species an alternative mode of long-distance dispersal via human-mediated transportation within and between continents. Over the last 25 years, there has been a global increase in both the distribution of $A$. aegypti and epidemic dengue virus activity (Jansen and Beebe, 2010). While climate influences the geographical distribution of this mosquito species, the close association of $A$. aegypti with humans and the domestic environment allows this species to persist in regions that may otherwise be unsuitable based on climatic factors alone. Quiescence dependent adult plasticity would greatly enhance this dispersal process by producing adults with an adaptive advantage in challenging and novel environments and allowing for sustained populations. For example a newly emerged female resulting from an extended quiescence egg would have greater lipid reserves available for longer host and oviposition seeking flights. Future work needs to determine if the plasticity described 
here is simply a norm of reaction (continuous range of phenotypes) or a case of alternative phenisim (discrete phenotypes).

Environmental conditions experienced during the mosquito's immature stages can subsequently affect adult phenotypes in a manner that may be important determinants of vector capacity (Merrit et al., 1992). This work has demonstrated that A. aegypti mosquitoes hatched from eggs that have undergone an extended quiescence in an urban environment are likely to exhibit greater vector capacity than mosquitoes hatched from short quiescence eggs. Laboratory studies have indicated that vector competence (i.e. the internal physiological factors that govern the infection of human pathogens in a mosquito) correlates with the quality of the larval environment and adversely affects larval survival, developmental rates and produces smaller adults with compromised fitness (Alto et al., 2008; Alto et al., 2012; Muturi et al. 2011; Telang et al. 2012). As a consequence, eggs that have undergone an extended quiescence in an urban environment could (a) contribute to increased vector capacity via increased dengue viral load of adult females and (b) have implications for vector control strategies. Following quiescence a mosquito population is in its most vulnerable state. A. aegypti adult mosquitoes reared from quiescent eggs and living in stressed urban environments produce far less viable offspring resulting in a seasonal population bottleneck, a phenomenon that mosquito control boards could use to their advantage.

\subsection{Acknowledgements}

We would like to thank Dr. Marcela Nouzova, Dr. Crisalejandra Rivera and especially Dr. Mark Clifton for the critical reading and feedback of the manuscript. This work was 
supported by NIH/NIGMS R25 GM061347 and the FIU MBRS RISE Biomedical Research Initiative to MHP. 


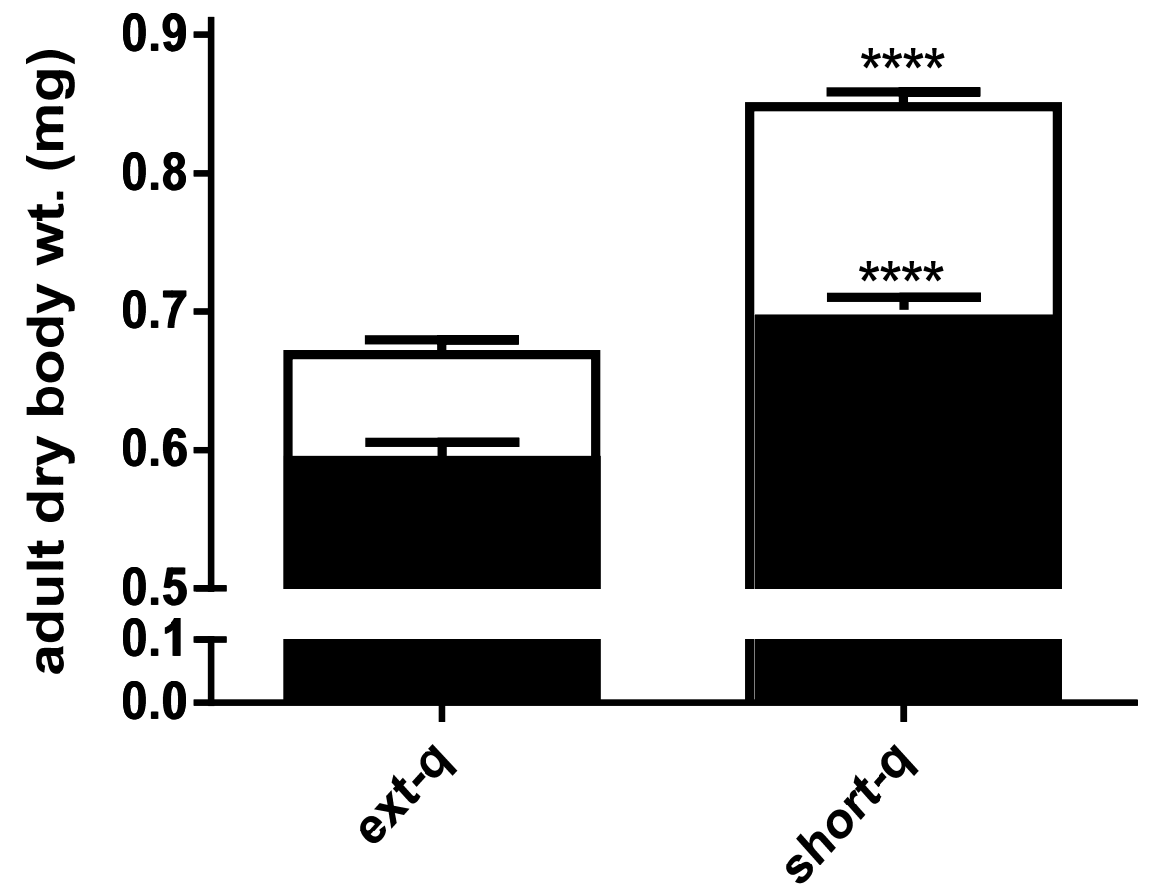

Fig. 17. Extended quiescence reduced female adult body mass: Extended quiescence significantly reduced female adult dry body mass at time of emergence by $21 \%$ (one tailed $\mathrm{t}$ test, $\mathrm{p}<0.0001, \mathrm{t}=11.83 \mathrm{df}=95)$. Larval metal stress reduced adult body size $17.3 \%$ in short quiescence females while only $11.4 \%$ in extended quiescence females representing a significant difference in metal physiology response between short quiescence and extended quiescence females (one tailed $t$ test, $p<0.05, t=7.083$ $\mathrm{df}=117$ )(Fig. 1). ( short-q=short quiescence, ext-q=extended quiescence) (white columns reared in clean water; black columns-reared with metal stress) $(\mathrm{n} \sim 50-60)$. 

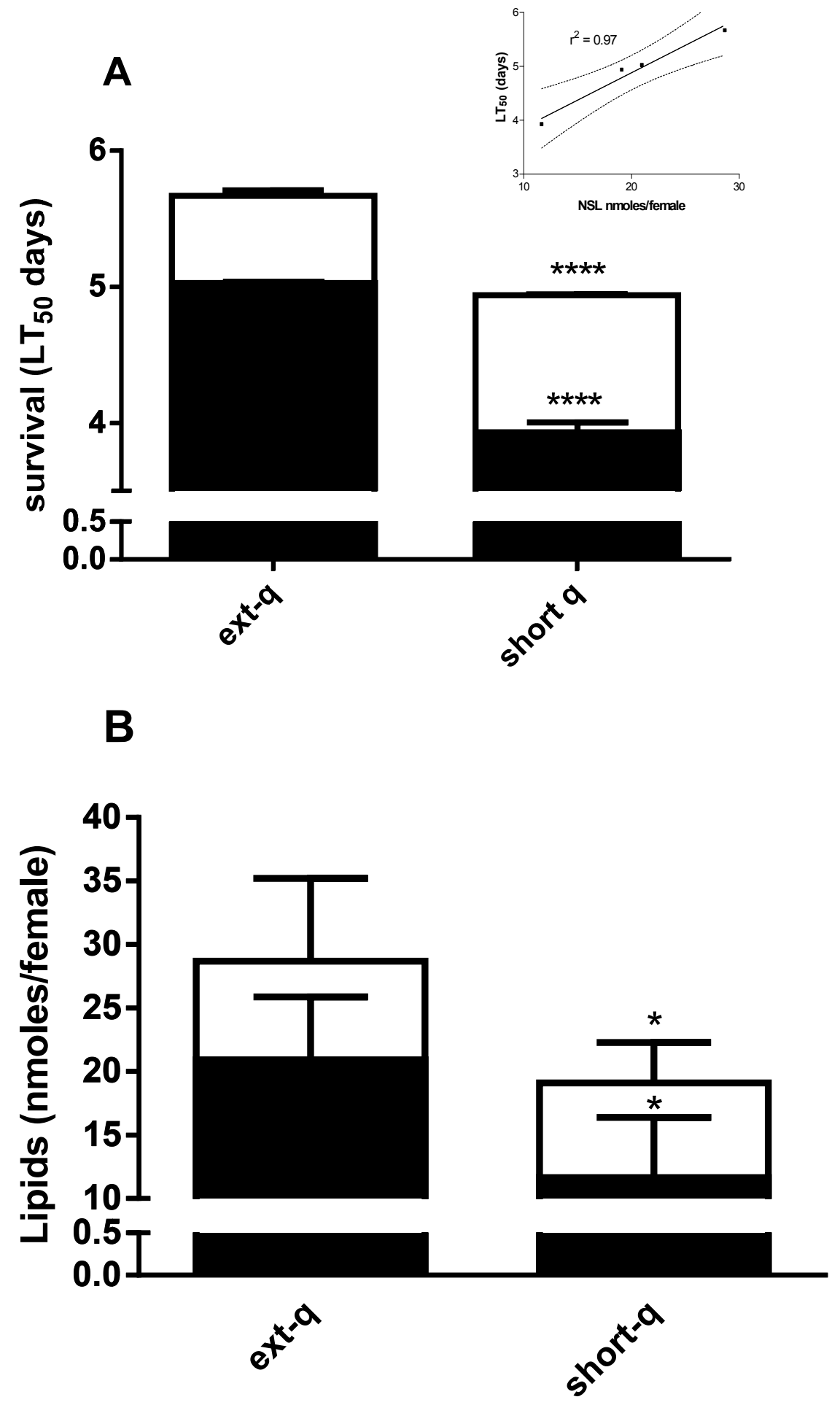
Fig. 18.(continued) Extended quiescence increased starvation tolerance of adult females: (A) Adult females reared from extended quiescence eggs exhibited $12.9 \%$ significantly greater survival time $\left(\mathrm{LT}_{50}\right)$ on a diet of only water than adult females reared from short quiescence eggs $(\mathrm{N}=45, \log$ rank, $\mathrm{p}<0.01)$. Larval metal stress reduced adult survival times significantly more in the short quiescence females $(20 \%)$ than in the extended quiescence females $(11.4 \%)$ when compared to their clean reared counterparts $(\mathrm{N}=45, \log$ rank, $\mathrm{p}<0.01$ ). (B) Adult females reared from extended quiescence eggs emerged with $33 \%$ significantly more neutral lipid reserves than adult females reared from short quiescence eggs ( $\mathrm{t}$ test, one tailed, $\mathrm{p}<0.05, \mathrm{t}=2.289$, $\mathrm{df}=4$ ); larval metal stress response reduced lipids significantly more in females from short quiescence than in females from extended quiescence, i.e., 20.0 vs. $11.4 \%$ respectively. ( $t$ test, one tailed, $p<0.05, t=2.350$, $\mathrm{df}=4$ ). Starvation tolerance and neutral lipids reserves are highly correlated (inset). (Legend follows Fig. 17). 


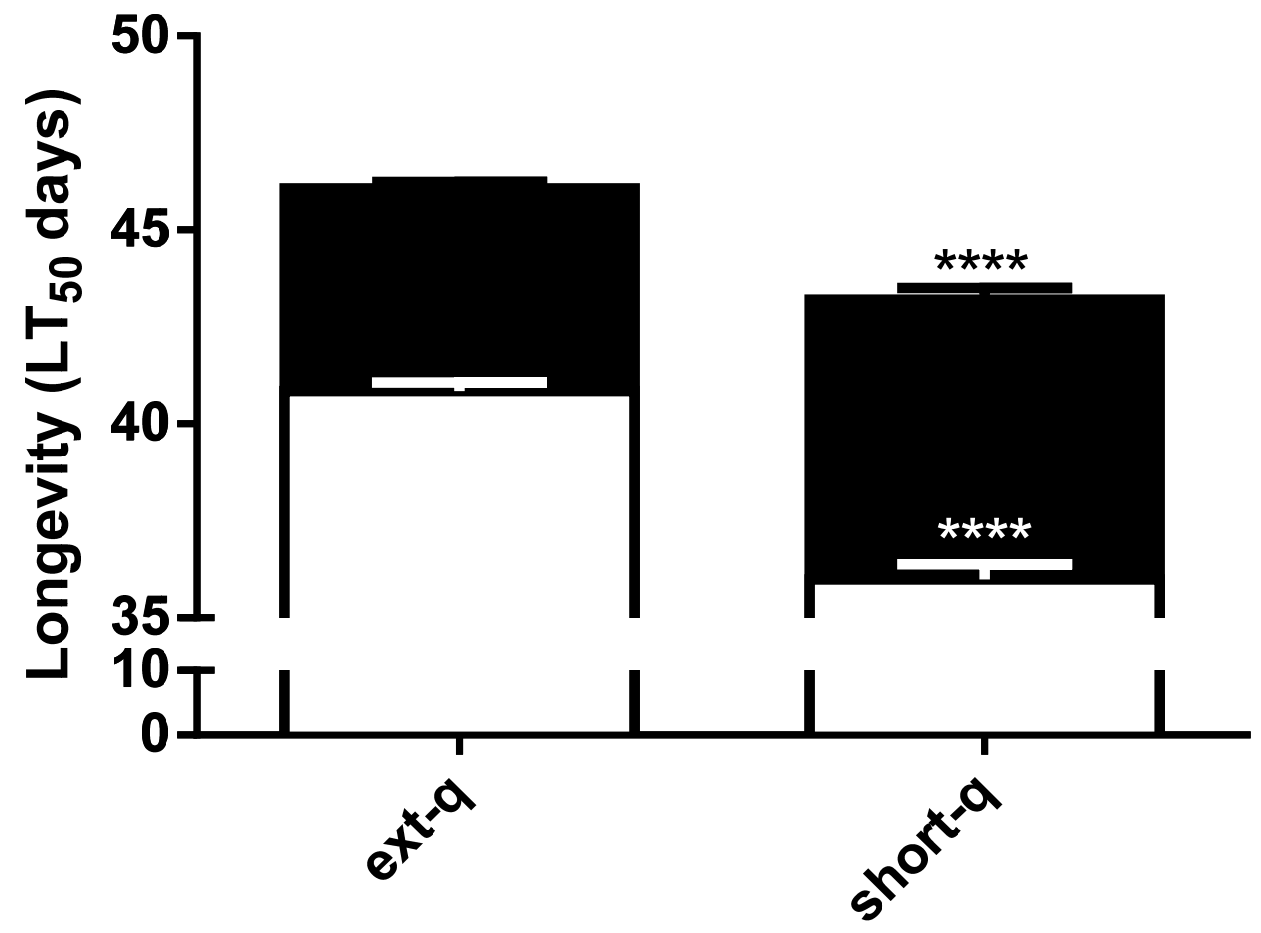

Fig. 19. Extended quiescence increased longevity of adult females: Adult female mosquitoes reared from extended quiescence eggs exhibited $10.2 \%$ significantly longer life span than adult female mosquitoes reared from short quiescence eggs (40.1 vs. 36.0 days) $(\mathrm{N}=45, \log$ rank, $\mathrm{p}<0.01)$. The hormetic effect of metal stress differed significantly between extended and short quiescence females, an increase of 12.8 vs. $20 \%$ respectively over their clean reared counterparts ( 46.1 vs. 43.2 days) (log rank test $\mathrm{p}<0.01)$ (Legend follows Fig. 17). 

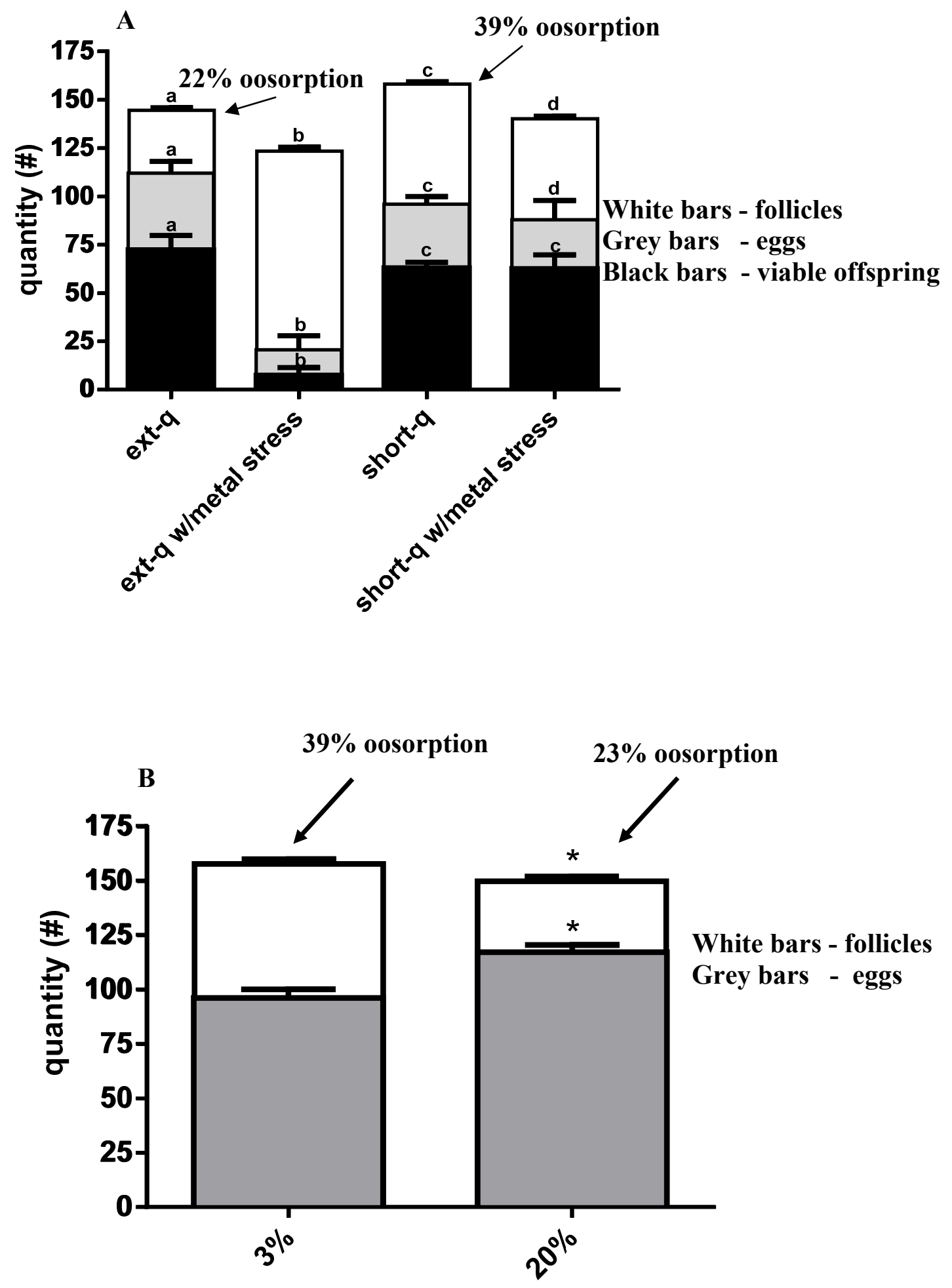
Fig. 20. (continued) Extended quiescence altered reproductive strategy: (A) Adult females reared from extended quiescence eggs developed significantly fewer follicles $(n=30$ pairs of ovaries from 15 females) (white bars) than females reared from short quiescence eggs ( $3 \%$ sucrose) but matured more eggs (gray bars) that resulted in more viable offspring (black bars) than their short quiescence counterparts. Although the reproductive profile among all four treatment combinations was significantly different, the metal stress response in extended quiescence females was dramatically altered (one way ANOVA $\mathrm{p}<0.05$, with Tukey test of multiple comparisons, $\mathrm{df}=58)(\mathrm{n}=15$ females). (B) Adults reared from short quiescence eggs on a diet of $20 \%$ sucrose resulted in less oosorption and more eggs being matured. Consequently, the number of eggs matured approximates the number of eggs developed by adults reared from extended quiescence eggs under the $3 \%$ sucrose diet $(\mathrm{n}=15)$ ( $\mathrm{t}$ test, one tailed, $\mathrm{p}<0.05)$. 


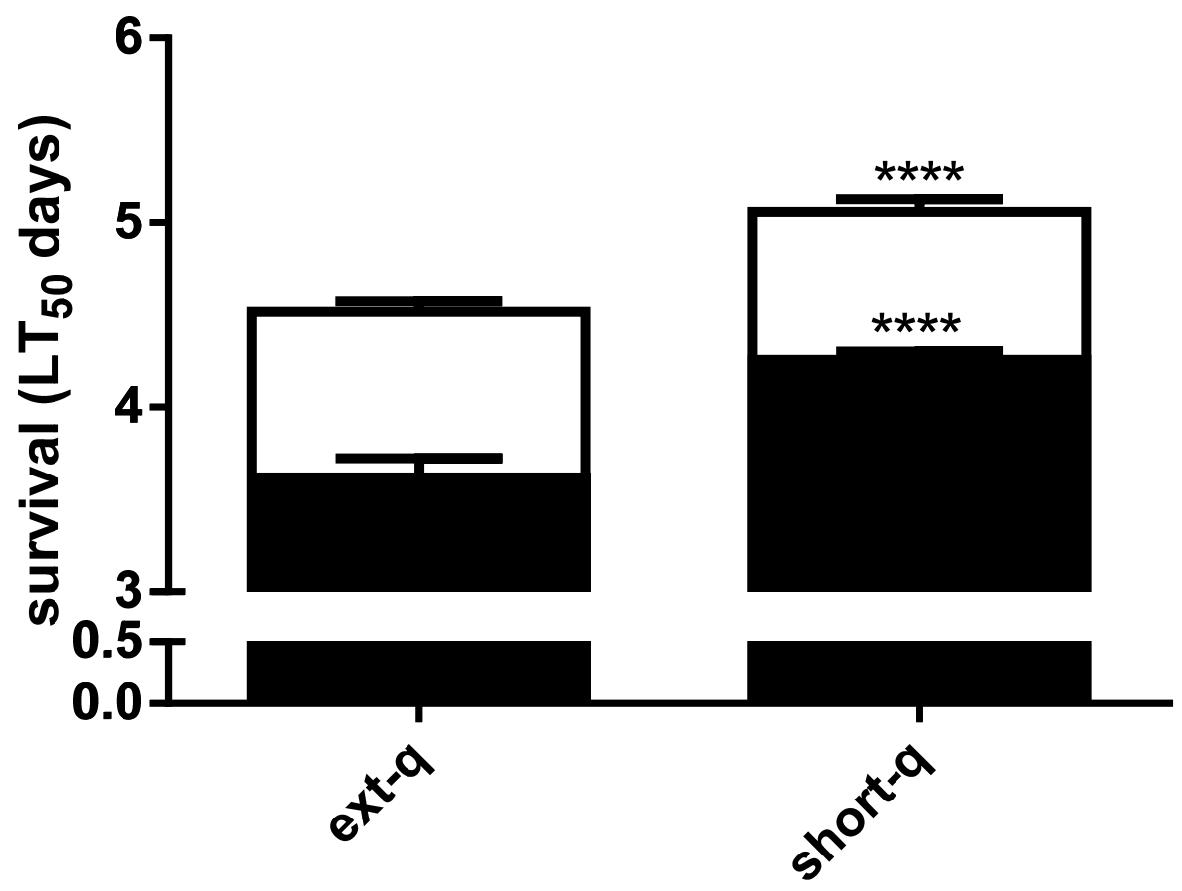

Fig. 21. Extended quiescence reduced tolerance to starvation of progeny first instar larvae: Extended quiescence resulted in maternal effects i.e., an $11.9 \%$ reduction in starvation tolerance of newly emerged first instar progeny ( 4.5 vs. $5.1 \mathrm{~d})$. The metal stress response differed significantly also; it was greater in the extended quiescence condition ( 20 vs. $16 \%$ ). ( $N=75, \log$ rank test, $\mathrm{p}<0.01$ ). (Legend follows Fig. 17) 


\subsection{References}

Alto, B. W., L. P. Lounibos, et al. (2008). "Larval competition alters susceptibility of adult Aedes mosquitoes to dengue infection." Proceedings of the Royal Society B: Biological Sciences 275(1633): 463-471.

Alto, B. W., E. J. Muturi, et al. (2012). "Effects of nutrition and density in Culex pipiens." Medical and Veterinary Entomology: 396-406.

Austad, S. N. (1989). "Life extension by dietary restriction in the bowl and doily spider, Frontinella pyramitela." Experimental Gerontology 24(1): 83-92.

Baldal, E. A., P. M. Brakefield, et al. (2006). "Multitrait evolution in lines of Drosophila melanogaster selected for increased starvation resistance: The role of metabolic rate and implications for the evolution of longevity " Evolution 60(7): 14351444 .

Barrera, R., M. Amador, et al. (2006). "Ecological Factors Influencing Aedes aegypti (Diptera: Culicidae) Productivity in Artificial Containers in Salinas, Puerto Rico." Journal of Medical Entomology 43(3): 484-92.

Barrera, R., M. Amador, et al. (2008). Unusual productivity of Aedes aegypti in septic tanks and its implications for dengue control. Medical and Veterinary Entomology. 22: 62-69.

Barsyte, D., D. A. Lovejoy, et al. (2001). "Longevity and heavy metal resistance in daf-2 and age-1 long-lived mutants of Caenorhabditis elegans." Journal of the Federation of American Societies for Experimental Biology 15(3): 627-634.

Blackmore, M. S. and C. C. Lord (2000). "The relationship between size and fecundity in Aedes albopictus." Journal of Vector Ecology 25(2 ): 212-217.

Boggs, C. L. (2009). "Understanding insect life histories and senescence through a resource allocation lens." Functional Ecology 23(1): 27-37.

Brakefield, P., J. Pijpe, et al. (2007). "Developmental plasticity and acclimation both contribute to adaptive responses to alternating seasons of plenty and of stress in \&lt;i\&gt;Bicyclus\&lt;/i\&gt; butterflies." Journal of Biosciences 32(3): 465-475.

Briegel, H. (1990). "Metabolic relationship between female body size, reserves, and fecundity of Aedes aegypti." Journal of Insect Physiology 36(3): 165-172.

Britton, J. S., W. K. Lockwood, et al. (2002). "Drosophila's Insulin/PI3-Kinase Pathway Coordinates Cellular Metabolism with Nutritional Conditions." Developmental Cell 2(2): 239-249. 
Broughton, S. J., M. D. W. Piper, et al. (2005). "Longer lifespan, altered metabolism, and stress resistance in Drosophila from ablation of cells making insulin-like ligands." Proceedings of the National Academy of Sciences 102(8): 3105-3110.

Caroci, A. S., Y. Li, et al. (2004). "Reduced juvenile hormone synthesis in mosquitoes with low teneral reserves reduces ovarian previtellogenic development in Aedes aegypti." Journal of Insect Physiology 207(15): 2685-2690.

Chippindale, A. K., A. M. Leroi, et al. (1993). "Phenotypic plasticity and selection in Drosophila life-history evolution. I. Nutrition and the cost of reproduction." Journal of Evolutionary Biology 6(2): 171-193.

Christophers, R., Ed. (1960). Aëdes aegypti (L.) the Yellow Fever Mosquito: its Life History, Bionomics and Structure, Cambridge University Press.

Clements, A. N., Ed. (1992). The Biology of Mosquitoes. London, Chapman and Hall.

Clifton, M. E. and F. G. Noriega (2011). "Nutrient limitation results in juvenile hormonemediated resorption of previtellogenic ovarian follicles in mosquitoes." Journal of Insect Physiology 57(9): 1274-1281.

Clifton, M. E. and F. G. Noriega (2012). "The fate of follicles after a blood meal is dependent on previtellogenic nutrition and juvenile hormone in Aedes aegypti." Journal of Insect Physiology 58(7): 1007-1019.

Denlinger, D. L. (1986). "Dormancy in Tropical Insects." Ann. Rev. Entornol. 31( ): 239-264.

Esperk, T., C. Stefanescu, et al. (2012). "Distinguishing between anticipatory and responsive plasticity in a seasonally polyphenic butterfly." Evolutionary Ecology: $1-18$.

Foster, W. A. (1995). "Mosquito sugar feeding and reproductive energetics" Annual Review of Entomology 40: 443-474.

Gary, R. E. and W. A. Foster (2001). "Effects of Available Sugar on the Reproductive Fitness and Vectorial Capacity of the Malaria Vector Anopheles gambiae (Diptera: Culicidae)." Journal of Medical Entomology 38(1): 22-28.

Gotthard, K., S. Nylin, et al. (1994). "Adaptive variation in growth rate: life history costs and consequences in the speckled wood butterfly, Pararge aegeria." Oecologia 99(3): 281-289. 
Hahn, D. A. and D. L. Denlinger (2007). "Meeting the energetic demands of insect diapause: Nutrient storage and utilization." Journal of Insect Physiology 53(8): 760-773.

Hahn, D. A. and D. L. Denlinger (2011). "Energetics of Insect Diapause." Annual Reviews of Entomology 56(1): 103-121.

Huber, K., Y. Ba, et al. (2008). Aedes aegypti in Senegal: Genetic Diversity and Genetic Structure of Domestic and Sylvatic Populations. Am J Trop Med Hyg. 79: 218229.

Jansen, C. C. and N. W. Beebe (2010). "The dengue vector Aedes aegypti: what comes next." Microbes and Infection 12(4): 272-279.

Joy, T. K., A. J. Arik, et al. (2010). "The impact of larval and adult dietary restriction on lifespan, reproduction and growth in the mosquito Aedes aegypti." Experimental Gerontology 45(9): 685-690.

Kirkwood, T. B. L. and S. N. Austad (2000). "Why do we age?" Nature 408(9): 233-238.

Mayr, E., Ed. (1963). Animal Species and Evolution, Cambridge: Belknap Press of Harvard University Press.

Merritt, R. W., R. H. Dadd, et al. (1992). Feeding Behavior, Natural Food, and Nutritional Relationships of Larval Mosquitoes. Entomology. 37: 349-374.

Mireji, P. O., J. Keating, et al. (2010). "Biological cost of tolerance to heavy metals in the mosquito Anopheles gambiae." Medical and Veterinary Entomology 24(1).

Moran, N. A. (1992). "The Evolutionary Maintenance of Alternative Phenotypes." The American Naturalist 139(5): 971-989.

Mori, A., T. Oda, et al. (1981). "Studies on the egg diapause and overwintering of Aedes albopictus in Nagasaki." Tropical Medicine 23(2): 79-90.

Munstermann, L. E. and L. M. Wasmuth, Eds. (1985). Handbook of Insect Rearing. Amsterdam, Elsevier

Muturi, E. J., Kim, et al. (2011). "Larval environmental stress alters Aedes aegypti competence for Sindbis virus " Tropical Medicine \& International Health 16(8): 955-964.

Perez, M. H. and F. G. Noriega (2012). "Aedes aegypti pharate first instar quiescence affects larval fitness and metal tolerance." Journal of Insect Physiology 58(6): 824-829. 
Pijpe, J., P. Brakefield, et al. (2007). "Phenotypic plasticity of starvation resistance in the butterfly, Bicyclus anynana." Evolutionary Ecology 21(5): 589-600.

Pook, C., C. Lewis, et al. (2009). "The metabolic and fitness costs associated with metal resistance in Nereis diversicolor." Marine Pollution Bulletin 58(7): 1063-1071.

Postma, J. F., A. Kleunen, et al. (1995). "Alterations in life-history traits of Chironomus riparius (diptera) obtained from metal contaminated rivers." Archives of Environmental Contamination and Toxicology 29(4): 469-475.

Powers, R. W., M. Kaeberlein, et al. (2006). "Extension of chronological life span in yeast by decreased TOR pathway signaling." Genes and Development 20(2): 174184.

Ronquillo, M. C. and W. R. Horsfall (1969). "Genesis of the reproductive system of mosquitoes. I. Female of Aedes stimulans (Walker)." Journal of Morphology 129(3): 249-280.

Servia, M., A. Péry, et al. (2006). "Effects of copper on energy metabolism and larval development in the midge, Chironomus riparius " Ecotoxicology 15(3): 229-240.

Simpson, S., G. Sword, et al. (2011). "Polyphenism in Insects." Current Biology 21(18): R738-R749.

Sinclair, D. A. (2005). "Toward a unified theory of caloric restriction and longevity regulation." Mechanisms of Ageing and Development 126(9): 987-1002.

Tatar, M., A. Kopelman, et al. (2001). "A Mutant Drosophila Insulin Receptor Homolog That Extends Life-Span and Impairs Neuroendocrine Function." Science 292(5514): 107-110.

Telang, A., A. Qayum, et al. (2012). "Larval nutritional stress affects vector immune traits in adult yellow fever mosquito Aedes aegypti (Stegomyia aegypti)." Medical and Veterinary Entomology 26(3): 271-281.

Vinogradova, E., V. Alekseev, et al. (2007). Diapause in Aquatic Insects, with Emphasis on Mosquitoes Diapause in Aquatic Invertebrates Theory and Human Use. H. J. Dumont and M. J. A. Werger, Springer Netherlands. 84: 83-113.

West-Eberhard, M. (2003). Developmental Plasticity and Evolution, Oxford University Press, USA.

Yaro, A. S., Traor, A. I., et al. (2012). "Dry season reproductive depression of Anopheles gambiae in the Sahel." Journal of Insect Physiology 58(8): 1050-1059. 


\section{Chapter 6: Conclusions and Final Remarks}

\subsection{Aedes aegypti pharate first instar quiescence: A distinctive life history trait with trade-offs and implications for urban vector biology}

Aedes aegypti is anthropophilic - adult females preferentially feed on humans and readily enter homes to feed, rest and seek refuge. Unlike many other mosquito species, $A$. aegypti is a day-biting mosquito and often feeds on multiple hosts during a single gonotrophic cycle (Christophers, 1960). Females preferentially lay eggs in man-made or artificial containers including water tanks, flower vases, potted plant bases, discarded tires, buckets pet water dishes or any other containers typically found around or inside the home (Barrera et al.,, 2006, 2008; Christophers, 1960; Hribar, et al., 2004). Eggs are laid near the water surface in containers, can withstand desiccation for up to one year and will hatch out immediately upon exposure to the appropriate stimulus (Russell et al., 2005). As a result of this life history trait, pharate first instar larvae can withstand months of dormancy inside the egg where they depend on stored reserves of maternal origin as demonstrated in chapter three. This adaptation allows this important pathogen vector to cope with fluctuations in water availability, however, $A$. aegypti experiences life history trade-offs associated with egg quiescence.

Being anthropophilic, A. aegypti resides in urban habitats that may contain significant metal pollution. My dissertation has shown that the duration of quiescence and the extent of nutritional depletion associated with it affects the physiology and survival of larvae that hatch in a suboptimal habitat, nutrient reserves decrease during pharate first instar quiescence and alter subsequent larval and adult fitness. The duration of quiescence 
also compromises metal tolerance physiology, most likely as a result of decreased nutritional reserves and metallothionein expression. The findings of chapter three have demonstrated that even low levels of environmentally relevant larval metal stress alter the parameters that determine vector capacity.

Vector capacity is the potential of a vector, such as $A$. aegypti, to transmit a pathogen as a function of total number of infective bites. An estimation of vector capacity is given by the Ross-Macdonald Equation; the Ross-Macdonald theory has played a central role in the development of research on mosquito-borne pathogen transmission and the development of strategies for mosquito-borne disease prevention (reviewed in Smith, 2009).

The Ross-Macdonald Equation:

Vector Capacity $=m a^{2} p^{n} b h /-\ln p$

where:

$m=$ vector density in relation to the host

$a=$ probability a vector feeds on a host in 1 day

$p=$ probability the vector will survive the extrinsic incubation period 1 day

$n=$ duration of the extrinsic incubation period (days)

$b=$ "vector competence" the proportion of vectors ingesting an infective meal that successfully become infected vector

$h=$ proportion of infected human population

$1 /(-\ln p)=$ duration of the vector's life, in days, after surviving the extrinsic incubation period

The Ross-MacDonald equation allows us to predict the effect of larval metal stress or pharate first instar quiescence on the vector capacity of an $A$. aegypti 
population. For example, the variables $\boldsymbol{m}$ and $\boldsymbol{p}$ are functions of adult survivorship. Decreased adult survivorship, a metal tolerance fitness cost, may result in decreased vector capacity (Mireji, et al., 2010). On the other hand, metal tolerance may increase vector capacity by increasing population abundances (variable $\boldsymbol{m}$ ) of mosquitoes in metal polluted environments. For example insecticide resistance increases the likelihood of mosquito-borne disease transmission by allowing mosquitoes to live in the presence of insecticides (McCarroll and Hemingway, 2002). Metal tolerance should also have a similar effect on vector capacity as insecticide resistance. Each of the variables responsible for estimating vector capacity are likely affected by larval metal stress and pharate first instar quiescence in a manner that will alter vector capacity. In addition, the decreased adult body size that has been demonstrated to be a direct result of larval metal stress and extended quiescence may increase the susceptibility of $A$. aegypti mosquitoes to dengue virus infection thereby increasing vector competence (variable b) (Alto, et al., 2008). All of these observations likely pertain to Aedes albopictus, the other vector of dengue fever (Alto et al., 2012).

Furthermore, the findings of this body of work demonstrated that extended pharate first instar quiescence can elicit a plastic response resulting in an adult phenotype distinct from adults reared from short quiescence eggs. Extended pharate first instar quiescence affects the performance and reproductive fitness of the adult female mosquito as well as the nutritional status of its progeny via maternal effects in an adaptive manner. Anticipatory phenotypic plasticity results as a consequence of the duration of pharate first instar quiescence such that alternative phenotypes may exist (or at the very least a reaction norm) for this mosquito with quiescence serving as a cue possibly signaling the 
environmental conditions that follow a dry period. These findings may explain, in part, $A$. aegypti's success as a vector and its wide geographic distribution and may have implications for its vector capacity and control. Pharate first instar quiescence, an adaptation that allows sylvatic populations to exploit transient water sources, has also inadvertently provided this vector with the ability to utilize the larval habitats not available to other mosquito vectors that are tied to a permanent water source (Christopher, 1960; Clements, 1992). Aedes aegypti once exploited man-made water vessels on sailing ships to colonize the new world now exploits the ample larval habitats we provide for it. This work has demonstrated that quiescence, a valuable adaptation for surviving dry periods, also cues an advantageous plastic response in which adult females emerging from quiescence are best suited to an environment following a dry period. Chapter five provides evidence for this advantageous plastic response by demonstrating that females derived from extended quiescence eggs are more stress tolerant and devote more resources to reproduction than their short quiescence counterparts. Egg quiescence, however, has a trade-off - larvae emerging from extended quiescence eggs are less tolerant of stress, a vulnerability that can be integrating into vector control strategies. Newly emerged mosquito larvae at the dry/wet transition are physiologically compromised, and therefore potentially more susceptible to larvicides than mosquito larvae hatched subsequently throughout the wet season.

Many questions remain unresolved regarding the evolutionary fitness costs and vector capacity of insects that are tolerant to pollutants. The effect of insecticide resistance on vector capacity has been extensively investigated, but not the effect of environmental contaminants. The knowledge acquired from this body of work can be 
applied to mosquito populations from urban habitats to direct efforts at their most vulnerable stages in the seasonal cycle and possibly to anticipate changes in disease transmission dynamics.

\subsection{Vector Capacity and the larval environment of $\boldsymbol{A}$. aegypti}

Vector competence studies have demonstrated that larval environmental conditions modify the susceptibility to infection and transmission of pathogens in aedine mosquito species (Alto and Lounibos, 2013 and references therein). Most studies on vector competence have focused on environmental conditions experienced by adults, but the larval environment shapes the phenotypes of adult mosquitoes and thereby alters vector competence for arthropod-borne viruses. Numerous studies have shown unequivocally that larval conditions such as nutrition, competition, temperature and insecticides alter adult vector competence for arboviruses. These effects show multiple and environmentally specific effects on barriers to virus infection, primarily the larval midgut; in addition alterations to blood feeding behavior have been shown. Future work needs to address the role of altered immune and stress response gene expression (Alto and Lounibos, 2013). Considering these observations and findings of Alto and Lounibos and other vector ecophysiologists it is not unreasonable to infer that ecologically relevant larval metal stress or extended first instar quiescence alone, or especially in combination, can significantly alter the vector capacity of A. aegypti. 


\subsection{A. aegypti and global climate change}

The potential impact of global climate change with its increased temperature and precipitation on pathogen transmission dynamics cannot be overlooked (Hopp and Foley, 2001). The number of cases of dengue fever in Asia and Latin America has steadily been on the increase throughout the last decades (WHO, 2012; PAHO, 2010). Until recently the risk of dengue fever in the continental United States was considered minimal because of: a) adequate infrastructure (storm sewers, mosquito abatement programs, etc.,) b) limited population density (planned urbanization) and c) limited personal exposure along with limited house permeability (windows with screens, central air conditioning, access to insect repellants) (Brunkyard, et al., 2007, 2008). Recently, however, the Florida Department of Health and the Center for Disease Control have reported cases of autochthonous dengue fever transmission in South Florida. In addition, the tremendous growth in international travel and trade increases the risk of importation of vector-borne diseases. Continued climate change with its increasing temperature and humidity, rising sea levels, increased flooding and ponding and its economic consequences can only increase the geographic distribution of $A$. aegypti and subsequently increase the potential of pathogen transmission. Demographic and sociologic factors also play a critical role in determining disease incidence, and it is likely that these diseases will cause major epidemics in the United States unless the public health infrastructure is not only well maintained but significantly improved, despite continued budget cuts. A. aegypti, with its aseasonal pharate first instar quiescence and potential ability to develop pollution 
tolerance is an arthropod vector poised to make dengue fever endemic in the sub-tropical United States. 


\subsection{References}

Alto, B. W. and L. P. Lounibos (2013). Vector competence for arboviruses in relation to the larval environment of mosquitoes. Ecology of parasite-vector interactions. C. J. M. Koenraadt, Springer: 81-101.

Alto, B. W., M. H. Reiskind, et al. (2008). Size Alters Susceptibility of Vectors to Dengue Virus Infection and Dissemination. 79: 688-695.

Barrera, R., M. Amador, et al. (2006). "Ecological Factors Influencing Aedes aegypti (Diptera: Culicidae) Productivity in Artificial Containers in Salinas, Puerto Rico." Population And Community Ecology 43(3): 484-92.

Barrera, R., M. Amador, et al. (2008). Unusual productivity of Aedes aegypti in septic tanks and its implications for dengue control. Medical and Veterinary Entomology. 22: 62-69.

Christophers, R., Ed. (1960). Aëdes aegypti (L.) the Yellow Fever Mosquito: its Life History, Bionomics and Structure, Cambridge University Press.

Clements, A. N., Ed. (1992). The Biology of Mosquitoes. London, Chapman and Hall.

Gubler, D. J., P. Reiter, et al. (2001). "Climate variability and change in the United States: potential impacts on vector-and rodent-borne diseases." Environmental Health Perspectives 109(Suppl 2): 223.

Hribar, L. J., J. J. Vlach, et al. (2004). "Mosquito larvae (Culicidae) and other Diptera associated with containers, storm drains, and sewage treatment plants in the Florida Keys, Monroe County, Florida." Florida Entomologist 87(2): 199-203.

McCarroll, L. and J. Hemingway (2002). "Can insecticide resistance status affect parasite transmission in mosquitoes?" Insect Biochemistry and Molecular Biology 32(10): 1345-1351.

Mireji, P. O., K. J, et al. (2010). "Biological cost of tolerance to heavy metals in the mosquito Anopheles gambiae" Medical and Veterinary Entomology 24(1).

Russell, R. C., C. E. Webb, et al. (2005). "Mark and release recapture study to measure dispersal of the mosquito Aedes aegypti in Cairns, Queensland, Australia." Medical and Veterinary Entomology 19(4): 451-457.

Smith, D. L., K. E. Battle, et al. (2009). "Ross, Macdonald, and a Theory for the Dynamics and Control of Mosquito-Transmitted Pathogens." PLoS Pathog 8(4) 
VITA

\author{
Mario H. Perez \\ Born, Staten Island, N. Y. \\ B.S. Biological Sciences, \\ College of Staten Island, \\ City University of New York \\ Ph. D. Biology \\ Florida International University
}

Professional Experience:

2005-2006 Laboratory Technician, Insect Physiology Laboratory, Florida International University, Department of Biological Sciences

2001-2004 Undergraduate Research Assistant, Center for Environmental Science, College of Staten Island

2001-2002 College Lab Technician, Biology Dept. College of Staten Island

2001 Field Research Assistant, Biology Dept. College of Staten Island

1997-2001 College Lab Technician, Chemistry Dept. College of Staten Island

Fellowships, Scholarships and Awards:

2012 Invited Student Speaker, Society of Vector Ecology Annual Conference, St.

Augustine Fl.

2010 National Institute of Allergy and Infectious Diseases (NIAID) Intramural NIAID

Research Opportunities (INRO) program award recipient

2007 Florida International University MBRS Research Initiative for Scientific

Enhancement (RISE) Program (NIGMS/NIH) (R25 GM061347)

2005 Florida International University Opportunities for Minorities Fellowship

Award2003 Excellence in Research Department of Biology Faculty Award

2003 Undergraduate Research Conference Award CSI/CUNY

2002 Undergraduate Summer Research Fellowship City University of New York, Office

of Grants and Research: Differences in prey capture of environmentally impacted

populations of the grass shrimp Palaemonetes pugio: Video analysis of prey capture

behavior

2002 Undergraduate Research Conference Award CSI/CUNY

2001 Undergraduate Summer Research Fellowship City University of New York, Office

of Grants and Research: Differences in prey capture of environmentally impacted populations of the grass shrimp Palaemonetes pugio: A laboratory transplant study. 2001 Undergraduate Research Conference Award CSI/CUNY 
2000 Undergraduate Summer Research Fellowship City University of New York, Office of Grants and Research: Site-specific disparity in successful prey capture of environmentally impacted populations of the grass shrimp Palaemonetes pugio: a preliminary examination of behavioral toxicity in Staten Island salt marshes

Selected Publications

Perez, Mario H. and Noriega Fernando G. (2013) Characterization of the sub-lethal larval metal stress response in the dengue vector Aedes aegypti, accepted, Physiological Entomology

Perez, Mario H. and Noriega Fernando G. (2013) Aedes aegypti pharate first instar quiescence: A case for reproductive polyphenisim. Journal of Insect Physiology, 59, 3 , $318-324$

Perez, Mario H. and Noriega Fernando G. (2012) Aedes aegypti pharate first instar quiescence affects larval fitness and metal tolerance. Journal of Insect Physiology, 58, 6, 824-829

Perez M.H., Wallace W.G. (2004) Differences in prey capture in grass shrimp, Palaemonetes pugio, collected along an environmental impact gradient. Arch. Environ. Contam. Toxicol. v46 p81-89

Conference/Meeting Participation:

2012 Society of Vector Ecology Annual Conference (oral presentation) Aedes aegypti first instar quiescence: a case for seasonal polyphenism?

2010 National Institute of Allergy and Infectious Diseases (NIAID) Intramural NIAID

Research Opportunities (INRO) program award participant

Teaching Experience:

Graduate: $\quad$ Instructor General Biology I and II laboratory Instructor Genetics laboratory

Undergraduate: Teaching Assistant Marine Biology, Teaching Assistant Chemistry 107 (Chemistry for non-science majors)

Professional Affiliations:

Associate member of Sigma Xi

Society of Toxicology and Environmental Chemistry (SETAC)

Society of Vector Ecology (SOVE) 